Preprint typeset in JHEP style - HYPER VERSION

hep-ph/0304168

SLAC-PUB-9675

UCLA $/ 03 /$ TEP $/ 10$

DESY 03-034

April, 2003

\title{
Two-Loop Helicity Amplitudes for Quark-Gluon Scattering in QCD and Gluino-Gluon Scattering in Supersymmetric Yang-Mills Theory
}

\author{
Zvi Bern* \\ Department of Physics and Astronomy \\ UCLA, Los Angeles, CA 90095-1547, USA \\ E-mail: bern@physics.ucla.edu \\ Abilio De Freitas \\ Deutsches Elektronen Synchrotron \\ DESY, D-15738 Zeuthen, Germany \\ E-mail: dfreitas@ifh.de

\section{Lance Dixon ${ }^{\dagger}$} \\ Stanford Linear Accelerator Center, Stanford University \\ Stanford, CA 94309, USA \\ E-mail: lance@slac.stanford.edu
}

\begin{abstract}
We present the two-loop QCD helicity amplitudes for quark-gluon scattering, and for quark-antiquark annihilation into two gluons. These amplitudes are relevant for next-to-next-to-leading order corrections to (polarized) jet production at hadron colliders. We give the results in the "t Hooft-Veltman and four-dimensional helicity (FDH) variants of dimensional regularization. The transition rules for converting the amplitudes between the different variants are much more intricate than for the previously discussed case of gluon-gluon scattering. Summing our two-loop expressions over helicities and colors, and converting to conventional dimensional regularization, gives results in complete agreement with those of Anastasiou, Glover, Oleari and Tejeda-Yeomans. We describe the amplitudes for $2 \rightarrow 2$ scattering in pure $N=1$ supersymmetric Yang-Mills theory, obtained from the QCD amplitudes by modifying the color representation and multiplicities, and verify supersymmetry Ward identities in the FDH scheme.
\end{abstract}

Keywords: QCD, NNLO Computations, Jets, Hadron Colliders.

Submitted to JHEP

Work supported in part by the Department of Energy contract DE-AC03-76SF00515. 


\section{Introduction}

Recent years have seen rapid progress in our ability to compute two-loop matrix elements with more than a single kinematic variable $[1,2,3,4,5,6,7,8,9,10,11]$. The progress has relied in part on new developments in loop integration $[12,13,14,15,16,17,18]$ and in understanding the infrared divergences of the theory [19]. The new two-loop amplitudes will be essential for reducing theoretical uncertainties in a number of physical quantities. (For a recent summary describing the various expected improvements see e.g. ref. [20].) In particular, in jet physics an important source of theoretical uncertainty is from missing higher order corrections to the widely used NLO calculations $[21,22,23]$, which have been crucial for matching theory to experiment. For the case of hadron colliders, one of the essential ingredients to performing next-to-next-to-leading order (NNLO) calculations are the parton distribution functions. Recently, global fits to the data for the parton distribution functions have been performed [24] within an approximate NNLO framework [25]. There are, however sizable uncertainties associated with the experimental input to the parton distribution functions [26]. Nevertheless, an exact NNLO computation of jet production rates would be very welcome. Besides reducing the scale uncertainties for jet rates, such a computation will allow a better understanding of energy flow within jets, as a jet may consist of up to three partons at this order. For very large momentum transfer, improvements can also be made by resumming threshold logarithms [27].

Besides the two loop matrix elements, an NNLO calculation of two jet production at hadron colliders requires the tree amplitudes for six external partons $[28,29]$ and the one-loop amplitudes for five external partons [30,31], which have been known for some time now. Anastasiou, Glover, Oleari, and Tejeda-Yeomans have provided the NNLO interferences of the two-loop amplitudes with the tree amplitudes, for all QCD four-parton processes, summed over all external helicities and colors $[4,5,6]$. The helicity amplitudes for $g g \rightarrow g g$ have also been computed, using the spinor helicity formalism [32]. In this paper, we present the $\bar{q} q \rightarrow g g$ and $q g \rightarrow q g$ two-loop helicity amplitudes. The four-quark helicity amplitudes will be presented elsewhere.

A useful property of helicity amplitudes is that they expose the full dependence on color and spin. Many formal properties of scattering amplitudes are simpler in a helicity basis and/or after color decomposition. Such properties include supersymmetry Ward identities [33, 34], collinear limits [29, 35, 36], and high-energy behavior [37, 38]. The full color dependence is also useful for understanding the structure of the infrared divergences $[19,9,39]$.

This additional formal information is not necessary for the main phenomenological application, NNLO jet production in collisions of unpolarized hadrons. On the other hand, the helicity amplitudes can have phenomenological applications for jet production in collisions of polarized protons, as are being carried out at the relativistic heavy ion collider (RHIC) at Brookhaven. This may, for example, help to determine the poorly-known polarized gluon distribution in the proton [40]. Theoretical predictions of the relevant observables require

* Research supported by the US Department of Energy under grant DE-FG03-91ER40662.

${ }^{\dagger}$ Research supported by the US Department of Energy under grant DE-AC03-76SF00515. 
scattering amplitudes for polarized partons. Currently, predictions are available through NLO [41]; the helicity amplitudes presented here are a prerequisite for improving the predictions to NNLO accuracy. Our results also serve as a check of the results of ref. [5] for unpolarized $q \bar{q} \rightarrow g g$ and $q g \rightarrow q g$ scattering.

While preparing our results for publication, we became aware of a similar computation being completed simultaneously [42]. Ref. [42] computes the one- and two-loop helicity amplitudes for $q \bar{q} \rightarrow g g$ and $q g \rightarrow q g$ scattering, in the 't Hooft-Veltman (HV) scheme [45] (see below). A slightly different method from ours is used to extract the helicity amplitudes. We have compared our results for the two-loop amplitudes (and also the one-loop amplitudes), through the finite terms required for NNLO cross sections, and we are in complete agreement.

In this paper we also describe the helicity amplitudes for gluino-gluon scattering in $N=1$ supersymmetric $S U(N)$ gauge theory. Due to supersymmetry Ward identities [33], these amplitudes are simply related to the $N=1$ gluon-gluon scattering amplitudes already presented in ref. [9]. The supersymmetric amplitudes are a close cousin of QCD amplitudes, differing only in fermion multiplicities and non-abelian charge assignments: The $N=1$ supersymmetric amplitude are obtained from the QCD amplitudes by replacing the quarks with a single adjoint color representation fermion, effectively converting them to a gluino superpartner of the gluon.

Because the scattering amplitudes possess both infrared and ultraviolet divergences, some care is required to ensure that the regularization procedure preserves supersymmetry. Several versions of dimensional regularization are commonly used for loop calculations in QCD. The widely used conventional dimensional regularization (CDR) scheme [43] breaks supersymmetry - it alters the balance between bosonic and fermionic states at order $\epsilon$, where $\epsilon=(4-D) / 2$ and $D$ is the number of dimensions. The CDR scheme is traditionally employed in calculations of amplitude interferences, such as in refs. [44, 3, 4, 5, 6]. In the helicity approach, the two commonly used schemes are the 't Hooft-Veltman (HV) scheme [45] and the four-dimensional helicity (FDH) scheme [46, 34]. These schemes differ in the number of polarization states for unobserved gluons. The 't Hooft-Veltman (HV) scheme [45] contains $2-2 \epsilon$ virtual gluon states (as does the CDR scheme), whereas the fourdimensional helicity (FDH) scheme $[46,34]$ assigns 2 states. The FDH scheme is related to dimensional reduction (DR) [47], but is more compatible with the helicity method, because it allows two transverse dimensions in which to define helicity. It is also possible to define a scheme, labeled by a parameter $\delta_{R}$, which interpolates between the $\mathrm{HV}\left(\delta_{R}=1\right)$ and FDH $\left(\delta_{R}=0\right)$ schemes. We shall present the $q \bar{q} \rightarrow g g$ and $q g \rightarrow q g$ amplitudes in this general $\delta_{R}$ scheme. A more detailed description of the differences between schemes, as well as a definition of the FDH scheme beyond one loop, has been given recently [34].

The supersymmetry preserving properties of the FDH scheme have been verified explicitly at two loops, for particular helicity configurations of four-gluon amplitudes that vanish at tree level $[34,9]$. In this paper, we explicitly verify that the FDH scheme preserves supersymmetry for the case of gluino-gluon scattering. Part of this check involves relating gluino-gluon amplitudes to gluon-gluon amplitudes, for helicity configurations which are non-vanishing at tree-level. Such a test is somewhat more stringent than previous 
ones $[9,48,34]$ because of the more intricate infrared divergences. The test additionally provides a nontrivial check on the calculation of the quark-gluon scattering amplitudes, as well as on the consistency of the FDH scheme.

In general, scattering amplitudes in massless QCD possess strong infrared (soft and collinear) divergences. Using dimensional regularization, the amplitudes generically contain poles in $\epsilon$ up to $1 / \epsilon^{4}$. Catani has organized these divergences into a compact form predicting their structure [19]. We use Catani's formula and color space notation to organize the helicity amplitudes into singular terms (which do contain order $\epsilon^{0}$ terms in their series expansion in $\epsilon$ ), plus finite remainders. The precise form of the $1 / \epsilon$ poles was not predicted a priori in ref. [19] for general processes at two loops. It is now clear, however, that these terms have a universal structure depending only on the external legs, based on explicit calculation $[3,4,5,6,7,8,9,10,11]$ and on matching to resummed results [39].

For the quark-gluon scattering amplitudes discussed here, ref. [5] previously computed the interference of the $1 / \epsilon$ pole terms with the tree amplitude, summed over all colors and helicities. Here we extract the full color and helicity dependence of the $1 / \epsilon$ pole terms. For the case of $g g \rightarrow g g$ amplitudes, a term independent of color and helicity was found [6], plus a second "surprise" term [9] with nontrivial color-dependence, which vanishes when the color-summed interference is performed. Here we confirm that a similar color-dependent term exists for the case of quark-gluon scattering amplitudes. We note that a term with similar color structure has been identified in contributions of one-loop factors for soft radiation to NNLO processes [49].

The conversion from one variant of dimensional regularization to another is well understood at one loop $[46,50,51]$. At two loops, the ultraviolet shift in the coupling constant has been calculated for the commonly used variants of dimensional regularization [34]. However, the infrared aspects have not yet been fully understood for arbitrary processes. The scheme dependence of $g g \rightarrow g g$ scattering amplitudes was studied in ref. [9]. There it was found that beginning at order $1 / \epsilon^{2}$ the functions appearing in Catani's infrared decomposition are actually scheme-dependent. Here we present the scheme dependence (dependence on $\delta_{R}$ ) for the $q \bar{q} \rightarrow g g$ amplitudes. The universal structure of the Catani formula for infrared divergences suggests that the conversion between schemes for any twoloop massless QCD amplitude would be controlled by the same set of functions that we uncover here and in ref. [9]. However, the $q \bar{q} \rightarrow g g$ scheme-dependence we find here is significantly more intricate than that found for $g g \rightarrow g g$, and we have not yet identified the general pattern.

The paper is organized as follows. In section 2 we review the infrared and color structure of one- and two-loop QCD amplitudes used to organize the amplitudes presented in this paper. In section 3 we describe the one-loop quark-gluon scattering amplitudes. Section 3.1 presents them in a form that is valid to all orders in $\epsilon$, in terms of integrals known through $\mathcal{O}\left(\epsilon^{2}\right)$. Their knowledge at this order is required for evaluating Catani's formula for the singular parts of the two-loop amplitudes through $\mathcal{O}\left(\epsilon^{0}\right)$. The finite oneloop remainder functions are given in section 3.2. The "square" of these functions, summed over colors and helicities, contributes to the NNLO cross section. In section 3.3, we describe how to carry out this sum, and compare the result to a similar sum performed in the CDR 
scheme $[5,52]$.

In section 4 we return to the two-loop amplitudes. The finite two-loop remainder functions are presented in section 4.1 and appendix A. Some auxiliary functions for describing the shift in the finite remainder functions are given in appendix B. The interference of the finite two-loop remainder functions with the tree amplitudes, summed over colors and helicities, also contributes to the NNLO cross section. In section 4.2 we describe the computation of this sum, and compare the results with those obtained in the CDR scheme in ref. [5].

In section 5 we discuss the $N=1$ super-Yang-Mills amplitudes obtained by modifying the QCD ones. The supersymmetry Ward identities are briefly reviewed in section 5.1. In sections 5.3 and 5.4 we discuss the results for the one- and two-loop gluino-gluon scattering amplitudes for pure $N=1$ supersymmetric Yang-Mills theory, after first reviewing the infrared structure for the theory in section 5.2. We verify that in the FDH scheme the two-loop amplitudes obey the expected supersymmetry Ward identities, which relate them to the gluon-gluon scattering amplitudes computed in ref. [9]. In section 6 we present our conclusions.

\section{Review of infrared and color structure}

In this section we review the structure of the infrared singularities of dimensionally regularized one- and two-loop QCD amplitudes, using Catani's color space notation [19], as a prelude to presenting the finite remainders of the one- and two-loop $q \bar{q} \rightarrow g g$ and $q g \rightarrow g q$ amplitudes.

The two processes considered in this paper are

$$
\begin{aligned}
& q\left(p_{1}, \lambda_{1}\right)+\bar{q}\left(p_{2}, \lambda_{2}\right) \rightarrow g\left(p_{3}, \lambda_{3}\right)+g\left(p_{4}, \lambda_{4}\right), \\
& q\left(p_{1}, \lambda_{1}\right)+g\left(p_{2}, \lambda_{2}\right) \rightarrow g\left(p_{3}, \lambda_{3}\right)+q\left(p_{4}, \lambda_{4}\right),
\end{aligned}
$$

using a "standard" (not "all-outgoing") convention for the external momentum $\left(p_{i}\right)$ and helicity $\left(\lambda_{i}\right)$ labeling. The Mandelstam variables are $s=\left(p_{1}+p_{2}\right)^{2}, t=\left(p_{1}-p_{4}\right)^{2}$, and $u=\left(p_{1}-p_{3}\right)^{2}$.

We use dimensional regularization to handle both ultraviolet and infrared singularities. We consider a continuous set of schemes, labeled by a parameter $\delta_{R}$ characterizing the number of virtual gluon degrees of freedom circulating in loops. (Because we are computing helicity amplitudes, the number of external gluon states is fixed at two.) Specifically, when the trace of the Minkowski metric is encountered, we set

$$
\eta_{\mu}^{\mu} \equiv D_{s} \equiv 4-2 \epsilon \delta_{R},
$$

corresponding to $2\left(1-\epsilon \delta_{R}\right)$ gluon states in the loop. Setting $\delta_{R}=1$ corresponds to the $\mathrm{HV}$ scheme, which is the most closely related to the CDR computation in ref. [5]. Setting $\delta_{R}=0$ corresponds to the FDH scheme, which has improved supersymmetry properties.

The CDR and HV schemes imply the same coupling constant, the standard $\overline{\mathrm{MS}}$ coupling, $\bar{\alpha}_{s}(\mu)$. The coupling in a general $\delta_{R}$ scheme is related to this coupling at NNLO 
by [34]

$$
\begin{aligned}
\alpha_{s}^{\delta_{R}}(\mu)=\bar{\alpha}_{s}(\mu)[1 & +\frac{C_{A}}{6}\left(1-\delta_{R}\right) \frac{\bar{\alpha}_{s}(\mu)}{2 \pi}+ \\
& +\left(\frac{C_{A}^{2}}{36}\left(1-\delta_{R}\right)^{2}+\frac{7 C_{A}^{2}-6 C_{F} T_{R} N_{f}}{12}\left(1-\delta_{R}\right)\right)\left(\frac{\bar{\alpha}_{s}(\mu)}{2 \pi}\right)^{2}+ \\
& \left.+\mathcal{O}\left(\left[\bar{\alpha}_{s}(\mu)\right]^{3}\right)\right]
\end{aligned}
$$

Henceforth we will suppress the $\delta_{R}$ index on $\alpha_{s}(\mu)$.

We work with ultraviolet renormalized amplitudes. The relation between the bare coupling $\alpha_{s}^{u}$ and renormalized coupling $\alpha_{s}(\mu)$, through two-loop order, is [19]

$$
\alpha_{s}^{u} \mu_{0}^{2 \epsilon} S_{\epsilon}=\alpha_{s}(\mu) \mu^{2 \epsilon}\left[1-\frac{\alpha_{s}(\mu)}{2 \pi} \frac{b_{0}}{\epsilon}+\left(\frac{\alpha_{s}(\mu)}{2 \pi}\right)^{2}\left(\frac{b_{0}^{2}}{\epsilon^{2}}-\frac{b_{1}}{2 \epsilon}\right)+\mathcal{O}\left(\alpha_{s}^{3}(\mu)\right)\right],
$$

where $\mu$ is the renormalization scale, $S_{\epsilon}=\exp [\epsilon(\ln 4 \pi+\psi(1))]$, and $\gamma=-\psi(1)=0.5772 \ldots$ is Euler's constant. The first two coefficients appearing in the beta function for QCD, or more generally $S U(N)$ gauge theory with $N_{f}$ flavors of massless fundamental representation quarks, are scheme-independent,

$$
b_{0}=\frac{11 C_{A}-4 T_{R} N_{f}}{6}, \quad b_{1}=\frac{17 C_{A}^{2}-\left(10 C_{A}+6 C_{F}\right) T_{R} N_{f}}{6},
$$

where $C_{A}=N, C_{F}=\left(N^{2}-1\right) /(2 N)$, and $T_{R}=1 / 2$. (Note that ref. [19] uses the notation $\beta_{0}=b_{0} /(2 \pi), \beta_{1}=b_{1} /(2 \pi)^{2}$.)

The perturbative expansion of the $q \bar{q} \rightarrow g g$ amplitude is

$$
\begin{aligned}
\mathcal{M}_{q \bar{q} \rightarrow g g}\left(\alpha_{s}(\mu), \mu ;\{p\}\right)=4 \pi \alpha_{s}(\mu) & {\left[\mathcal{M}_{q \bar{q} \rightarrow g g}^{(0)}(\mu ;\{p\})+\right.} \\
& +\frac{\alpha_{s}(\mu)}{2 \pi} \mathcal{M}_{q \bar{q} \rightarrow g g}^{(1)}(\mu ;\{p\})+ \\
& \left.+\left(\frac{\alpha_{s}(\mu)}{2 \pi}\right)^{2} \mathcal{M}_{q \bar{q} \rightarrow g g}^{(2)}(\mu ;\{p\})+\mathcal{O}\left(\alpha_{s}^{3}(\mu)\right)\right],
\end{aligned}
$$

where $\mathcal{M}_{q \bar{q} \rightarrow g g}^{(L)}(\mu ;\{p\})$ is the $L^{\text {th }}$ loop contribution. The same type of expansion holds for the $q g \rightarrow g q$ amplitude. Equation (2.5) is equivalent to the following $\overline{\mathrm{MS}}$ renormalization prescriptions at one and two loops,

$$
\begin{aligned}
& \mathcal{M}_{q \bar{q} \rightarrow g g}^{(1)}=S_{\epsilon}^{-1} \mathcal{M}_{q \bar{q} \rightarrow g g}^{(1) \text { unren }}-\frac{b_{0}}{\epsilon} \mathcal{M}_{q \bar{q} \rightarrow g g}^{(0)}, \\
& \mathcal{M}_{q \bar{q} \rightarrow g g}^{(2)}=S_{\epsilon}^{-2} \mathcal{M}_{q \bar{q} \rightarrow g g}^{(2) \text { unren }}-2 \frac{b_{0}}{\epsilon} S_{\epsilon}^{-1} \mathcal{M}_{q \bar{q} \rightarrow g g}^{(1) \text { unren }}+\left(\frac{b_{0}^{2}}{\epsilon^{2}}-\frac{b_{1}}{2 \epsilon}\right) \mathcal{M}_{q \bar{q} \rightarrow g g}^{(0)} .
\end{aligned}
$$

The infrared divergences of renormalized one- and two-loop $n$-point amplitudes are given by [19],

$$
\begin{aligned}
\left|\mathcal{M}_{n}^{(1)}(\mu ;\{p\})\right\rangle_{\mathrm{RS}}= & \boldsymbol{I}^{(1)}(\epsilon, \mu ;\{p\})\left|\mathcal{M}_{n}^{(0)}(\mu ;\{p\})\right\rangle_{\mathrm{RS}}+\left|\mathcal{M}_{n}^{(1) \operatorname{fin}}(\mu ;\{p\})\right\rangle_{\mathrm{RSS}} \\
\left|\mathcal{M}_{n}^{(2)}(\mu ;\{p\})\right\rangle_{\mathrm{RS} .}= & \boldsymbol{I}^{(1)}(\epsilon, \mu ;\{p\})\left|\mathcal{M}_{n}^{(1)}(\mu ;\{p\})\right\rangle_{\mathrm{RS} .} \\
& +\boldsymbol{I}_{\mathrm{R} . \mathrm{S}}^{(2)}(\epsilon, \mu ;\{p\})\left|\mathcal{M}_{n}^{(0)}(\mu ;\{p\})\right\rangle_{\mathrm{R} . \mathrm{S}}+\left|\mathcal{M}_{n}^{(2) \operatorname{fin}}(\mu ;\{p\})\right\rangle_{\mathrm{R} \text {.S }}
\end{aligned}
$$




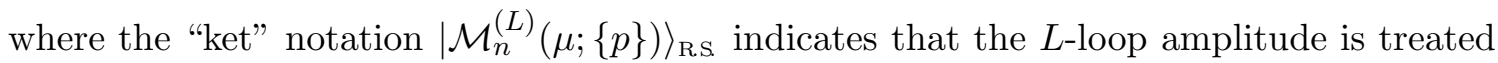
as a vector in color space. The actual amplitude is extracted via

$$
\mathcal{M}_{n}\left(1^{a_{1}}, \ldots, n^{a_{n}}\right) \equiv\left\langle a_{1}, \ldots, a_{n} \mid \mathcal{M}_{n}\left(p_{1}, \ldots, p_{n}\right)\right\rangle
$$

where the $a_{i}$ are color indices. The subscript R.s. indicates that a quantity depends on the choice of regularization and renormalization scheme. The divergences of $\mathcal{M}_{n}^{(1)}$ are encoded in the color operator $\boldsymbol{I}^{(1)}$, while those of $\mathcal{M}_{n}^{(2)}$ also involve the scheme-dependent operator $\boldsymbol{I}_{\mathrm{RS} \text {. }}^{(2)}$.

In QCD, the operator $\boldsymbol{I}^{(1)}$ is given by

$$
\boldsymbol{I}^{(1)}(\epsilon, \mu ;\{p\})=\frac{1}{2} \frac{e^{-\epsilon \psi(1)}}{\Gamma(1-\epsilon)} \sum_{i=1}^{n} \sum_{j \neq i}^{n} \boldsymbol{T}_{i} \cdot \boldsymbol{T}_{j}\left[\frac{1}{\epsilon^{2}}+\frac{\gamma_{i}}{\boldsymbol{T}_{i}^{2}} \frac{1}{\epsilon}\right]\left(\frac{\mu^{2} e^{-i \lambda_{i j} \pi}}{2 p_{i} \cdot p_{j}}\right)^{\epsilon}
$$

where $\lambda_{i j}=+1$ if $i$ and $j$ are both incoming or outgoing partons, and $\lambda_{i j}=0$ otherwise. The color charge $\boldsymbol{T}_{i}=\left\{T_{i}^{a}\right\}$ is a vector with respect to the generator label $a$, and an $S U(N)$ matrix with respect to the color indices of the outgoing parton $i$. For external gluons $T_{c b}^{a}=i f^{c a b}$, so $\boldsymbol{T}_{i}^{2}=C_{A}=N$, and

$$
\gamma_{g}=\frac{11 C_{A}-4 T_{R} N_{f}}{6}
$$

For external fermions, the ratio

$$
\frac{\gamma_{q}}{\boldsymbol{T}_{i}^{2}}=\frac{3}{2}
$$

is independent of the representation. For quarks, $\boldsymbol{T}_{i}^{2}=C_{F}=\left(N^{2}-1\right) /(2 N)$; for gluinos $\boldsymbol{T}_{i}^{2}=C_{A}=N$.

The operator $\boldsymbol{I}_{\mathrm{RS} \text {. }}^{(2)}$ is given by [19]

$$
\begin{aligned}
\boldsymbol{I}_{\mathrm{R} . \mathrm{S}}^{(2)}(\epsilon, \mu ;\{p\})= & -\frac{1}{2} \boldsymbol{I}^{(1)}(\epsilon, \mu ;\{p\})\left(\boldsymbol{I}^{(1)}(\epsilon, \mu ;\{p\})+\frac{2 b_{0}}{\epsilon}\right)+ \\
& +\frac{e^{+\epsilon \psi(1)} \Gamma(1-2 \epsilon)}{\Gamma(1-\epsilon)}\left(\frac{b_{0}}{\epsilon}+K_{\mathrm{R} . \mathrm{S}}\right) \boldsymbol{I}^{(1)}(2 \epsilon, \mu ;\{p\})+ \\
& +\boldsymbol{H}_{\mathrm{R} . \mathrm{S}}^{(2)}(\epsilon, \mu ;\{p\}),
\end{aligned}
$$

where the coefficient $K_{\mathrm{RS} .}$ depends on $\delta_{R}$ and is given by $[19,9]$

$$
K_{\mathrm{RS} .}=\left[\frac{67}{18}-\frac{\pi^{2}}{6}-\left(\frac{1}{6}+\frac{4}{9} \epsilon\right)\left(1-\delta_{R}\right)\right] C_{A}-\frac{10}{9} T_{R} N_{f}
$$

The function $\boldsymbol{H}_{\mathrm{R} \text {.S }}^{(2)}$ contains only single poles, and splits into two types of terms,

$$
\boldsymbol{H}^{(2)}(\epsilon)=\frac{e^{-\epsilon \psi(1)}}{4 \epsilon \Gamma(1-\epsilon)}\left(\frac{\mu^{2}}{-s}\right)^{2 \epsilon}\left(\left(2 H_{q}^{(2)}+2 H_{g}^{(2)}\right) \mathbf{1}+\hat{\boldsymbol{H}}^{(2)}\right) .
$$


From the calculations performed here we find that the term proportional to the identity matrix in color space 1 contains the constants $H_{g}^{(2)}$ and $H_{q}^{(2)}$, given by

$$
\begin{aligned}
H_{q}^{(2)}= & \left(\frac{13}{2} \zeta_{3}-\frac{23}{48} \pi^{2}+\frac{245}{216}\right) C_{A} C_{F}+\left(-6 \zeta_{3}+\frac{\pi^{2}}{2}-\frac{3}{8}\right) C_{F}^{2}+\left(\frac{\pi^{2}}{12}-\frac{25}{54}\right) C_{F} T_{R} N_{f} \\
& +\left(-\frac{4}{3} C_{A} C_{F}+\frac{1}{2} C_{F}^{2}+\frac{1}{6} C_{F} T_{R} N_{f}\right)\left(1-\delta_{R}\right), \\
H_{g}^{(2)}= & \left(\frac{\zeta_{3}}{2}+\frac{11}{144} \pi^{2}+\frac{5}{12}\right) C_{A}^{2}-\left(\frac{\pi^{2}}{36}+\frac{58}{27}\right) C_{A} T_{R} N_{f}+C_{F} T_{R} N_{f}+\frac{20}{27} T_{R}^{2} N_{f}^{2} \\
& +\left(-\frac{11}{36} C_{A}^{2}+\frac{1}{9} C_{A} T_{R} N_{f}\right)\left(1-\delta_{R}\right) .
\end{aligned}
$$

This term survives the sum over colors, and the expressions for $H_{g}^{(2)}$ and $H_{q}^{(2)}$ in the HV scheme $\left(\delta_{R}=1\right)$ agree, as expected, with previous color-summed results in the CDR scheme $[4,5,6,53]$. The $H_{g}^{(2)}$ coefficient agrees for all $\delta_{R}$ with that extracted from the $g g \rightarrow g g$ helicity amplitudes [9].

The second term in $\boldsymbol{H}^{(2)}(\epsilon)$ has exactly the same type of nontrivial color and kinematic dependence found in the $g g \rightarrow g g$ helicity amplitudes [9], namely

$$
\hat{\boldsymbol{H}}^{(2)}=-4 \ln \left(\frac{-s}{-t}\right) \ln \left(\frac{-t}{-u}\right) \ln \left(\frac{-u}{-s}\right) \times\left[\boldsymbol{T}_{1} \cdot \boldsymbol{T}_{2}, \boldsymbol{T}_{2} \cdot \boldsymbol{T}_{3}\right],
$$

with $\ln ((-s) /(-t)) \rightarrow \ln s-\ln (-t)-i \pi$ in the $s$-channel, etc. (It might seem that the operator $\boldsymbol{T}_{1} \cdot \boldsymbol{T}_{2}$ is somewhat ambiguous for the $q \bar{q} \rightarrow g g$ process (2.1), since the $q \bar{q}$ and $g g$ pairs have different color quantum numbers. However, the difference between the $q \bar{q}$ and $g g \boldsymbol{T}_{1} \cdot \boldsymbol{T}_{2}$ operators is proportional to the identity — it is a difference of Casimirs - so the commutator is unambiguous.)

In ref. [9] it was observed for the $g g \rightarrow g g$ amplitudes that the second term in eq. (2.18) is independent of the helicity configuration, and is a nontrivial commutator matrix in color space. (The possibility of nontrivial color structure in $\boldsymbol{H}^{(2)}(\epsilon)$ was pointed out in ref. [19].) Now we can see that $\hat{\boldsymbol{H}}^{(2)}$ is also independent of whether the external lines are quarks or gluons, which buttresses the suggestion [9] that it is related to soft, not collinear, virtual contributions. A similar color structure emerges in a general analysis of the contributions of one-loop factors for soft radiation to NNLO processes [49]. Because of the commutator structure, $\hat{\boldsymbol{H}}^{(2)}$ vanishes when sandwiched between tree amplitudes, after performing a sum over colors; hence it drops out of the color-summed interference of the two-loop amplitudes with the tree amplitudes [9].

To proceed further, we wish to introduce an explicit color basis for the amplitudes, and also remove certain overall spinor product factors. To do the latter, we take the set of independent helicity configurations $h$ to be

$$
\begin{array}{ll}
h=1: & q\left(p_{1},+\right)+\bar{q}\left(p_{2},-\right) \rightarrow g\left(p_{3},+\right)+g\left(p_{4},+\right), \\
h=2: & q\left(p_{1},+\right)+\bar{q}\left(p_{2},-\right) \rightarrow g\left(p_{3},-\right)+g\left(p_{4},+\right), \\
h=3: & q\left(p_{1},+\right)+g\left(p_{2},-\right) \rightarrow g\left(p_{3},+\right)+q\left(p_{4},+\right),
\end{array}
$$




\begin{tabular}{|c|c|c|}
\hline Amplitude & Value of $h$ & Permutation \\
\hline \hline$q_{1}^{+} \bar{q}_{2}^{-} \rightarrow g_{3}^{+} g_{4}^{+}$ & $h=1$ & - \\
\hline$q_{1}^{+} \bar{q}_{2}^{-} \rightarrow g_{3}^{-} g_{4}^{+}$ & $h=2$ & - \\
\hline$q_{1}^{+} \bar{q}_{2}^{-} \rightarrow g_{3}^{+} g_{4}^{-}$ & $h=2$ & $\{t \leftrightarrow u\}$ \\
\hline$q_{1}^{+} \bar{q}_{2}^{-} \rightarrow g_{3}^{-} g_{4}^{-}$ & $h=1$ & $\{t \leftrightarrow u\}$ \\
\hline \hline$q_{1}^{+} g_{2}^{+} \rightarrow g_{3}^{+} q_{4}^{+}$ & $h=5$ & - \\
\hline$q_{1}^{+} g_{2}^{-} \rightarrow g_{3}^{+} q_{4}^{+}$ & $h=3$ & - \\
\hline$q_{1}^{+} g_{2}^{+} \rightarrow g_{3}^{-} q_{4}^{+}$ & $h=3$ & - \\
\hline$q_{1}^{+} g_{2}^{-} \rightarrow g_{3}^{-} q_{4}^{+}$ & $h=4$ & - \\
\hline
\end{tabular}

Table 1: Relations between a general helicity configuration containing $q\left(p_{1},+\right)$ and the five presented in the text, eqs. (2.22)-(2.26).

$$
\begin{array}{ll}
h=4: & q\left(p_{1},+\right)+g\left(p_{2},-\right) \rightarrow g\left(p_{3},-\right)+q\left(p_{4},+\right), \\
h=5: & q\left(p_{1},+\right)+g\left(p_{2},+\right) \rightarrow g\left(p_{3},+\right)+q\left(p_{4},+\right) .
\end{array}
$$

Other configurations are simply related to these by symmetries. For example, the $q\left(p_{1},-\right)$ amplitudes are obtained by parity (P); the $\bar{q} g \rightarrow g \bar{q}$ amplitudes are related to $q g \rightarrow g q$ by charge conjugation $(\mathrm{C}) ; q\left(p_{1},+\right)+\bar{q}\left(p_{2},-\right) \rightarrow g\left(p_{3},-\right)+g\left(p_{4},-\right)$ is related to process $(2.22)$ by $\mathrm{CP}$; and $q\left(p_{1},+\right)+g\left(p_{2},+\right) \rightarrow g\left(p_{3},-\right)+q\left(p_{4},+\right)$ is related to process $(2.24)$ by time reversal (T). Maintaining helicity conservation on the quark line removes half of the configurations. Applying parity to the remaining configurations, in order to let the helicity of $q\left(p_{1}\right)$ be positive, there are four configurations each for $q \bar{q} \rightarrow g g$ and $q g \rightarrow g q$. Table 1 relates these eight configurations to the five represented by eqs. (2.22)-(2.26).

Helicity-dependent, phase-containing factors arise because we evaluate the amplitudes in the spinor helicity formalism [32]. For $h=2,4,5$, we remove a factor $S_{h}$ related to the tree amplitude. For $h=1,3$, the tree amplitude vanishes, and we remove a factor related to the one-loop amplitude. We define

$$
\begin{aligned}
& S_{1}=-i \frac{\langle 13\rangle[34]}{\langle 23\rangle\langle 34\rangle}, \quad S_{2}=i \frac{\langle 13\rangle^{3}\langle 23\rangle}{\langle 12\rangle\langle 23\rangle\langle 34\rangle\langle 41\rangle}, \\
& S_{3}=-i \frac{\langle 13\rangle[32]}{\langle 23\rangle\langle 34\rangle}, \quad S_{4}=i \frac{\langle 13\rangle^{3}\langle 43\rangle}{\langle 12\rangle\langle 23\rangle\langle 34\rangle\langle 41\rangle}, \quad S_{5}=i \frac{\langle 12\rangle^{3}\langle 42\rangle}{\langle 12\rangle\langle 23\rangle\langle 34\rangle\langle 41\rangle} \cdot(2.2
\end{aligned}
$$

The spinor inner products $[32,29]$ are $\langle i j\rangle=\left\langle i^{-} \mid j^{+}\right\rangle$and $[i j]=\left\langle i^{+} \mid j^{-}\right\rangle$, where $\left|i^{ \pm}\right\rangle$are massless Weyl spinors of momentum $k_{i}$, labeled with the sign of the helicity. They are anti-symmetric, with norm $|\langle i j\rangle|=|[i j]|=\sqrt{s_{i j}}$, where $s_{i j}=2 k_{i} \cdot k_{j}$. The squares of the prefactors $S_{h}$ enter polarized cross sections,

$$
\begin{aligned}
\left|S_{1}\right|^{2} & =\frac{u}{t}, & \left|S_{2}\right|^{2}=\frac{u^{3}}{s^{2} t}, \\
\left|S_{3}\right|^{2}=-\frac{u}{s}, & \left|S_{4}\right|^{2}=-\frac{u^{3}}{s t^{2}}, & \left|S_{5}\right|^{2}=-\frac{s u}{t^{2}},
\end{aligned}
$$

where we have included a minus sign for all the $q g \rightarrow g q$ cases to ensure positive tree-level cross sections. 
The color decomposition of the amplitudes (or their finite parts, according to eqs. (2.10) and $(2.11))$ reads

$$
\mathcal{M}_{h}^{(L)}=S_{h} \times \sum_{c=1}^{3} \operatorname{Tr}^{[c]} \times M_{h}^{(L),[c]}, \quad h=1,2,3,4,5
$$

where

$$
\operatorname{Tr}^{[1]}=\left(T^{a_{3}} T^{a_{4}}\right)^{i_{2}} \overline{\bar{l}}_{1}, \quad \operatorname{Tr}^{[2]}=\left(T^{a_{4}} T^{a_{3}}\right)^{i_{2}} \bar{\imath}_{1}, \quad \operatorname{Tr}^{[3]}=\delta^{a_{3} a_{4}} \delta_{\bar{\imath}_{1}}^{i_{2}}, \quad h=1,2,
$$

and

$$
\operatorname{Tr}^{[1]}=\left(T^{a_{3}} T^{a_{2}}\right)^{i_{\bar{l}_{1}}}, \quad \operatorname{Tr}^{[2]}=\left(T^{a_{2}} T^{a_{3}}\right)^{i_{\bar{l}_{1}}}, \quad \operatorname{Tr}^{[3]}=\delta^{a_{2} a_{3}} \delta_{\bar{\nu}_{1}}^{i_{4}}, \quad h=3,4,5 .(2.3
$$

Here $T^{a}$ are $S U(N)$ generators in the fundamental representation, normalized according to the convention typically used in helicity amplitude calculations, $\operatorname{tr}\left(T^{a} T^{b}\right)=\delta^{a b}$. (The $T^{a}$ used in this color decomposition should not be confused with the $T_{i}^{a}$ appearing in $\boldsymbol{I}^{(1)}$, whose representation depends on the external line; nor should they be confused with the generators for the quark representation, which have the more "standard" normalization, $T_{R}=1 / 2$, as mentioned above.) The amplitude components depend on the Mandelstam variables, $M_{h}^{(L),[c]} \equiv M_{h}^{(L),[c]}(s, t, u)$. Often we will suppress the kinematic arguments, unless a permutation of them is involved.

Note that we use a charge-conjugated fundamental index for quarks and anti-quarks in the initial state, i.e. $\bar{\imath}_{1}$ for $q_{1}$, and $i_{2}$ for $\bar{q}_{2}$. Also, amplitudes related to $h=1,2,3,4,5$ by charge conjugation $(\mathrm{C})$ will naturally have the fundamental indices charge conjugated.

In the basis (2.30) for $q \bar{q} \rightarrow g g$, the matrix $\boldsymbol{I}^{(1)}$ is

$$
\begin{aligned}
& \boldsymbol{I}^{(1)}(\epsilon)=-\frac{e^{-\epsilon \psi(1)}}{\Gamma(1-\epsilon)} \times \\
& \times\left(\begin{array}{ccc}
\left(-\frac{1}{2 N} \xi_{q}+\frac{N}{2} \xi_{g}\right) \mathrm{S}+N \xi_{q g} \mathrm{~T} & \xi_{q g}(\mathrm{~T}-\mathrm{U}) \\
0 & \left(-\frac{1}{2 N} \xi_{q}+\frac{N}{2} \xi_{g}\right) \mathrm{S}+N \xi_{q g} \mathrm{U} & -\xi_{q g}(\mathrm{~T}-\mathrm{U}) \\
\xi_{q g}(\mathrm{~S}-\mathrm{U}) & \xi_{q g}(\mathrm{~S}-\mathrm{T}) & \left(\frac{V}{2 N} \xi_{q}+N \xi_{g}\right) \mathrm{S}
\end{array}\right)
\end{aligned}
$$

where

$$
\begin{array}{ll}
\mathrm{S}=\left(\frac{\mu^{2}}{-s}\right)^{\epsilon}, & \mathrm{T}=\left(\frac{\mu^{2}}{-t}\right)^{\epsilon}, \quad \mathrm{U}=\left(\frac{\mu^{2}}{-u}\right)^{\epsilon}, \\
\xi_{q}=\frac{1}{\epsilon^{2}}+\frac{3}{2 \epsilon}, & \xi_{g}=\frac{1}{\epsilon^{2}}+\frac{b_{0}}{N \epsilon}, \quad \xi_{q g}=\frac{1}{2}\left(\xi_{q}+\xi_{g}\right), \\
V=N^{2}-1 . &
\end{array}
$$

The corresponding operator for $q g \rightarrow g q$ in the basis (2.31) is obtained by exchanging $\mathrm{S}$ and $\mathrm{T}$ in eq. (2.32).

The tree amplitudes in the color basis $(2.30),(2.31)$ are given by $M_{h}^{(0),[c]}$, where

$$
M_{1}^{(0),[c]}=M_{3}^{(0),[c]}=0, \quad c=1,2,3,
$$




$$
\begin{array}{llrl}
M_{h}^{(0),[3]}=0, & & h=1,2,3,4,5, \\
M_{2}^{(0),[1]}=1, & M_{2}^{(0),[2]}=\frac{t}{u}, \\
M_{4}^{(0),[1]}=1, & M_{4}^{(0),[2]}=\frac{s}{u}, \\
M_{5}^{(0),[1]}=1, & M_{5}^{(0),[2]}=\frac{s}{u} .
\end{array}
$$

A typical partonic cross section requires an amplitude interference, summed over all external colors. Such interferences are evaluated in the color bases (2.30), (2.31) as

$$
I_{\lambda_{1} \lambda_{2} \lambda_{3} \lambda_{4}}^{\left(L, L^{\prime}\right)} \equiv\left\langle\mathcal{M}_{\lambda_{1} \lambda_{2} \lambda_{3} \lambda_{4}}^{(L)} \mid \mathcal{M}_{\lambda_{1} \lambda_{2} \lambda_{3} \lambda_{4}}^{\left(L^{\prime}\right)}\right\rangle=\sum_{c, c^{\prime}=1}^{3} M_{\lambda_{1} \lambda_{2} \lambda_{3} \lambda_{4}}^{(L),[c] *} \mathcal{C C}_{c c^{\prime}} M_{\lambda_{1} \lambda_{2} \lambda_{3} \lambda_{4}}^{\left(L^{\prime}\right),\left[c^{\prime}\right]}
$$

where the symmetric matrix $\mathcal{C C}_{c c^{\prime}} \equiv \sum_{\text {colors }} \operatorname{Tr}^{[c] *} \operatorname{Tr}^{\left[c^{\prime}\right]}$ is

$$
\mathcal{C C}=\frac{V}{N}\left(\begin{array}{ccc}
V & -1 & N \\
-1 & V & N \\
N & N & N^{2}
\end{array}\right)
$$

One can use table 1 to convert from the $\lambda_{1} \lambda_{2} \lambda_{3} \lambda_{4}$ helicity configuration label to the label $h \in\{1,2,3,4,5\}$. The unpolarized partonic cross section is obtained from the helicity sum

$$
\bar{I}^{\left(L, L^{\prime}\right)} \equiv \sum_{\lambda_{i}= \pm 1} I_{\lambda_{1} \lambda_{2} \lambda_{3} \lambda_{4}}^{\left(L, L^{\prime}\right)}
$$

after the usual averaging over initial spins and inclusion of flux factors. For example, the helicity sum for the tree-level cross sections for $q \bar{q} \rightarrow g g$ and $q g \rightarrow g q$, constructed from eqs. (2.36) and (2.28) in either the HV or FDH scheme, i.e., for any $\delta_{R}$, are

$$
\begin{aligned}
& \bar{I}_{q \bar{q} \rightarrow g g}^{(0,0)}=2 V \frac{t^{2}+u^{2}}{t u}\left(N \frac{t^{2}+u^{2}}{s^{2}}-\frac{1}{N}\right), \\
& \bar{I}_{q g \rightarrow g q}^{(0,0)}=-2 V \frac{s^{2}+u^{2}}{s u}\left(N \frac{s^{2}+u^{2}}{t^{2}}-\frac{1}{N}\right) .
\end{aligned}
$$

\section{One-loop amplitudes}

The one-loop amplitudes for $q \bar{q} \rightarrow g g$ were first evaluated through $\mathcal{O}\left(\epsilon^{0}\right)$ as an interference with the tree amplitude in the CDR scheme [44]. Later they were evaluated as helicity amplitudes in the $\mathrm{HV}$ and $\mathrm{FDH}$ (or $\overline{\mathrm{DR}}$ ) schemes [50].

Because $\boldsymbol{I}^{(1)}$ contains terms of order $1 / \epsilon^{2}$, the $\boldsymbol{I}^{(1)}\left|\mathcal{M}^{(1)}\right\rangle_{\mathrm{RS}}$ term in the infrared decomposition (2.11) of the two-loop $q \bar{q} \rightarrow g g$ amplitudes requires the series expansions of the one-loop amplitudes through $\mathcal{O}\left(\epsilon^{2}\right)$. In section 3.1 we present the all-order results in the color bases (2.30), (2.31), with the normalizations implicit in eq. (2.7), in terms of integral functions whose series expansions are known to the requisite order $[2,3]$.

In ref. [9] we showed that $\mathcal{O}(\epsilon)$ terms in one-loop amplitudes such as $\mathcal{M}_{q \bar{q} \rightarrow g g}^{(1)}$ are not required for the construction of a numerical NNLO program, once such terms have 
been subtracted from $\mathcal{M}_{q \bar{q} \rightarrow g g}^{(2)}$ in the framework of ref. [19]. Thus we need only present explicit formulae for the finite remainders $\mathcal{M}_{q \bar{q} \rightarrow g g}^{(1) \mathrm{fin}}$ of the one-loop amplitudes, after ultraviolet renormalization (2.8) and subtraction of infrared divergences (2.10). We do this in section 3.2 , for a general $\delta_{R}$ scheme.

In section 3.3 we give a formula for the contribution of the finite remainders $\mathcal{M}_{q \bar{q} \rightarrow g g}^{(1) \mathrm{fin}}$ to the NNLO cross section. We compare the result of evaluating the formula in the HV scheme to other computations in both the HV and CDR schemes [52].

Actually, the formulas (4.3) and (4.4) for converting the two-loop finite remainders $\mathcal{M}_{q \bar{q} \rightarrow g g}^{(2) f i n}$ from one scheme to another are most compactly presented in terms of the $\delta_{R^{-}}$ dependent parts of the one-loop amplitudes at order $\epsilon$; these quantities are presented in appendix B.

\subsection{All orders in $\epsilon$ QCD amplitudes}

We now present the renormalized one-loop $q \bar{q} \rightarrow g g$ amplitudes in the color bases $(2.30)$, (2.31), with the normalizations implicit in eq. (2.7), in a form valid to all orders in $\epsilon$.

At one loop the crossing properties of the amplitudes are relatively simple, so we present the explicit values of the helicity amplitudes for the process $q \bar{q} \rightarrow g g$. The $q g \rightarrow g q$ process may be obtained from these by crossing the antiquark into the final state, and gluon 4 into the initial state,

$$
\begin{aligned}
& M_{3}^{(1),[c]}(s, t, u)=M_{1}^{(1),[c]}(t, s, u), \\
& M_{4}^{(1),[c]}(s, t, u)=M_{2}^{(1),[c]}(t, s, u), \\
& M_{5}^{(1),[c]}(s, t, u)=\frac{s}{u} M_{2}^{(1),\left[c^{\prime}\right]}(t, u, s),
\end{aligned}
$$

where $M_{h}^{(L),[c]}$ is defined in eq. (2.29) with the color bases (2.30) and (2.31) using the helicity configurations $h$ defined in eqs. (2.22)-(2.26). The factor of $s / u$ in the third relation accounts for the permutation of the $S_{2}$ prefactor in eq. (2.27). The color label $c^{\prime}$ needed for the $h=5$ case (3.3) is given by $c^{\prime}=2,1,3$ for $c=1,2,3$, respectively. After crossing, appropriate analytic continuations are required to bring each function into the physical region; for the finite parts for convenience we will give the explicit forms in the different analytic regions.

It is convenient to give the amplitudes in terms of "primitive" amplitudes, which are color-stripped building blocks for full amplitudes in any color representation. For the case of one-loop amplitudes with a single external fermion pair, the explicit relations between the primitive amplitudes and the color decomposed amplitudes were presented in ref. [31]. Here we quote the results for the decomposition and then present the primitive amplitudes. The same amplitudes, but in a form valid only through $\mathcal{O}\left(\epsilon^{0}\right)$, as needed in an NLO calculation, may be found in refs. [50,31].

The first coefficient in the color basis (2.30) for $q \bar{q} \rightarrow g g$ at one loop is expressed in terms of (unrenormalized) primitive amplitudes as [31]

$$
M_{1}^{(1),[1]}(s, t, u)=N A^{L}\left(1_{q}^{+}, 2_{\bar{q}}^{-}, 3_{g}^{+}, 4_{g}^{+}\right)-\frac{1}{N} A^{R}\left(1_{q}^{+}, 2_{\bar{q}}^{-}, 3_{g}^{+}, 4_{g}^{+}\right)+
$$




$$
\begin{aligned}
& +N_{f} A^{L,[1 / 2]}\left(1_{q}^{+}, 2_{\bar{q}}^{-}, 3_{g}^{+}, 4_{g}^{+}\right), \\
M_{2}^{(1),[1]}(s, t, u)= & N A^{L}\left(1_{q}^{+}, 2_{\bar{q}}^{-}, 3_{g}^{-}, 4_{g}^{+}\right)-\frac{1}{N} A^{R}\left(1_{q}^{+}, 2_{\bar{q}}^{-}, 3_{g}^{-}, 4_{g}^{+}\right)+ \\
& +N_{f} A^{L,[1 / 2]}\left(1_{q}^{+}, 2_{\bar{q}}^{-}, 3_{g}^{-}, 4_{g}^{+}\right)-\frac{b_{0}}{\epsilon} M_{2}^{(0),[1]},
\end{aligned}
$$

where, as defined in ref. [31], the $L$ and $R$ superscripts refer to whether the external fermion line turns "left" or "right" upon entering a diagram. The "[1/2]" designation on the last primitive amplitude represents the subset of contributions with a closed spin $1 / 2$ fermion. We continue to label the helicities using the "standard" convention with legs 1 and 2 incoming and legs 3 and 4 outgoing.

The coefficient of the second color factor for $h=1$ in the basis (2.30) is obtained from the first one by permuting kinematics and helicity labels,

$$
M_{1}^{(1),[2]}(s, t, u)=-\frac{t}{u} M_{1}^{(1),[1]}(s, u, t),
$$

where the $-t / u$ prefactor accounts for the implicit permutations of the removed $S_{h}$ prefactors in eq. (2.27). The coefficient of the second color factor for $h=2$ is given by

$$
\begin{aligned}
M_{2}^{(1),[2]}(s, t, u)= & N A^{L}\left(1_{q}^{+}, 2_{\bar{q}}^{-}, 3_{g}^{+}, 4_{g}^{-}\right)-\frac{1}{N} A^{R}\left(1_{q}^{+}, 2_{\bar{q}}^{-}, 3_{g}^{+}, 4_{g}^{-}\right)+ \\
& +N_{f} A^{L,[1 / 2]}\left(1_{q}^{+}, 2_{\bar{q}}^{-}, 3_{g}^{+}, 4_{g}^{-}\right)-\frac{b_{0}}{\epsilon} M_{2}^{(0),[2]}
\end{aligned}
$$

For the third color structure in eq. (2.30), we have

$$
\begin{aligned}
M_{1}^{(1),[3]}(s, t, u)= & A^{L}\left(1_{q}^{+}, 2_{\bar{q}}^{-}, 3_{g}^{+}, 4_{g}^{+}\right)+A^{R}\left(1_{q}^{+}, 2_{\bar{q}}^{-}, 3_{g}^{+}, 4_{g}^{+}\right)-\frac{t}{u} A^{L}\left(1_{q}^{+}, 2_{\bar{q}}^{-}, 4_{g}^{+}, 3_{g}^{+}\right)- \\
& -\frac{t}{u} A^{R}\left(1_{q}^{+}, 2_{\bar{q}}^{-}, 4_{g}^{+}, 3_{g}^{+}\right)+2 \frac{s}{u} A^{R}\left(1_{q}^{+}, 4_{g}^{+}, 2_{\bar{q}}^{-}, 3_{g}^{+}\right), \\
M_{2}^{(1),[3]}(s, t, u)= & A^{L}\left(1_{q}^{+}, 2_{\bar{q}}^{-}, 3_{g}^{-}, 4_{g}^{+}\right)+A^{R}\left(1_{q}^{+}, 2_{\bar{q}}^{-}, 3_{g}^{-}, 4_{g}^{+}\right)+\frac{t}{u} A^{L}\left(1_{q}^{+}, 2_{\bar{q}}^{-}, 4_{g}^{+}, 3_{g}^{-}\right)+ \\
& +\frac{t}{u} A^{R}\left(1_{q}^{+}, 2_{\bar{q}}^{-}, 4_{g}^{+}, 3_{g}^{-}\right)+2 \frac{s}{u} A^{R}\left(1_{q}^{+}, 4_{g}^{+}, 2_{\bar{q}}^{-}, 3_{g}^{-}\right) .
\end{aligned}
$$

where again the ratios of kinematic invariants (including signs) account for the permutations of the extracted the overall prefactors (2.27) from the amplitudes with standard ordering of legs $\left(1_{q}, 2_{\bar{q}}, 3_{g}, 4_{g}\right)$.

In order to compress the notation a little, we shall suppress the labels of legs in the explicit formulas for the primitive amplitudes. We always take them to be ordered $(1,2,3,4)$ in the following formulas, and apply permutations as required by eqs. (3.6)-(3.9). That is, define

$$
\begin{aligned}
& A_{q^{\lambda_{1}} \bar{q}^{\lambda_{2}} g^{\lambda_{3}} g^{\lambda_{4}}}^{L} \equiv A^{L}\left(1_{q}^{\lambda_{1}}, 2_{\bar{q}}^{\lambda_{2}}, 3_{g}^{\lambda_{3}}, 4_{g}^{\lambda_{4}}\right), \\
& A_{q^{\lambda_{1}} \bar{q}^{\lambda_{2}} g^{\lambda_{3}} g^{\lambda_{4}}}^{R} \equiv A^{R}\left(1_{q}^{\lambda_{1}}, 2_{\bar{q}}^{\lambda_{2}}, 3_{g}^{\lambda_{3}}, 4_{g}^{\lambda_{4}}\right), \\
& A_{q^{\lambda_{1}} \bar{q}^{\lambda_{2}} g^{\lambda_{3}} g^{\lambda_{4}}}^{L,[/ 2]} \equiv A^{L,[1 / 2]}\left(1_{q}^{\lambda_{1}}, 2_{\bar{q}}^{\lambda_{2}}, 3_{g}^{\lambda_{3}}, 4_{g}^{\lambda_{4}}\right), \\
& A_{q^{\lambda_{1}} g^{\lambda_{2}} \bar{q}^{\lambda_{3}} g^{\lambda_{4}}}^{R} \equiv A^{R}\left(1_{q}^{\lambda_{1}}, 2_{g}^{\lambda_{2}}, 3_{\bar{q}}^{\lambda_{3}}, 4_{g}^{\lambda_{4}}\right) .
\end{aligned}
$$


Then, for example, $A^{L}\left(1_{q}^{+}, 2_{\bar{q}}^{-}, 4_{g}^{+}, 3_{g}^{-}\right)=\left.A_{q^{+} \bar{q}^{-} g^{+} g^{-}}^{L}\right|_{t \leftrightarrow u}$.

In this notation, the explicit values of the independent primitive helicity amplitudes — in terms of a set of scalar integral functions - are:

$$
\begin{aligned}
& A_{q^{+} \bar{q}^{-} g^{+} g^{+}}^{L}=\frac{\epsilon\left(1-\epsilon \delta_{R}\right) t}{4 u}\left[\frac{\epsilon((5-2 \epsilon) s+2 t)}{(1-\epsilon)(1-2 \epsilon)(3-2 \epsilon)} \operatorname{Tri}^{(4)}(s)+\right. \\
& \left.+\frac{\epsilon s}{(1-\epsilon)(1-2 \epsilon)} \operatorname{Tri}^{(4)}(t)-s \operatorname{Box}^{(6)}(s, t)\right] \text {, } \\
& A_{q^{+} \bar{q}^{-} g^{+} g^{+}}^{R}=-A_{q^{+} \bar{q}^{-} g^{+} g^{+}}^{L}-\left(1-\epsilon \delta_{R}\right) A_{q^{+} \bar{q}^{-} g^{+} g^{+}}^{L,[1 / 2]}, \\
& A_{q^{+} \bar{q}^{-} g^{+} g^{+}}^{L,[1 / 2]}=\frac{\epsilon^{2} t}{2(1-\epsilon)(1-2 \epsilon)(3-2 \epsilon)} \operatorname{Tri}^{(4)}(s), \\
& A_{q^{+} \bar{q}^{-} g^{-} g^{+}}^{L}=\frac{\left(1-\epsilon \delta_{R}\right) s t}{4 u^{2}}\left[\frac { \epsilon } { ( 1 - \epsilon ) ( 1 - 2 \epsilon ) } \left(((3-\epsilon) s+t) \operatorname{Tr}^{(4)}(s)+\right.\right. \\
& \left.\left.+((1-\epsilon) s-t) \operatorname{Tri}^{(4)}(t)\right)-(2-\epsilon) s \operatorname{Box}^{(6)}(s, t)\right]+ \\
& +\frac{1}{2 u}\left[\frac { 1 } { 1 - 2 \epsilon } \left(s((1-2 \epsilon) s+t) \operatorname{Tri}^{(4)}(s)+\right.\right. \\
& \left.+2 t((1-\epsilon) s+(1-2 \epsilon) t) \operatorname{Tri}^{(4)}(t)\right)+ \\
& \left.+\left((1-2 \epsilon)\left(s^{2}+2 t^{2}\right)+(1-4 \epsilon) s t\right) \operatorname{Box}^{(6)}(s, t)\right] \text {, } \\
& A_{q^{+} \bar{q}^{-} g^{-} g^{+}}^{R}=-\frac{\left(1-\epsilon \delta_{R}\right) s t}{4 u^{2}}\left[\frac { \epsilon } { ( 1 - \epsilon ) ( 1 - 2 \epsilon ) } \left(((1+\epsilon) s-(1-2 \epsilon) t) \operatorname{Tri}^{(4)}(s)-\right.\right. \\
& \left.-((1-\epsilon) s+(3-2 \epsilon) t) \operatorname{Tri}^{(4)}(t)\right)- \\
& \left.-(\epsilon s-2(1-\epsilon) t) \operatorname{Box}^{(6)}(s, t)\right]+ \\
& +\frac{s}{2 u}\left[\frac{1}{1-2 \epsilon}\left(((1-2 \epsilon) s+t) \operatorname{Tri}^{(4)}(s)+2 \epsilon t \operatorname{Tri}^{(4)}(t)\right)+\right. \\
& \left.+((1-2 \epsilon) s-t) \operatorname{Box}^{(6)}(s, t)\right] \\
& A_{q^{+} \bar{q}^{-} g^{-} g^{+}}^{L,[1 / 2]}=0 \\
& A_{q^{+} \bar{q}^{-} g^{+} g^{-}}^{L}=-\frac{\epsilon\left(1-\epsilon \delta_{R}\right) s}{4 u}\left[\frac{1}{(1-\epsilon)(1-2 \epsilon)}\left(((1-\epsilon) s+t) \operatorname{Tri}^{(4)}(s)+\epsilon t \operatorname{Tri}^{(4)}(t)\right)+\right. \\
& \left.+s \operatorname{Box}^{(6)}(s, t)\right]-
\end{aligned}
$$




$$
\begin{aligned}
& -\frac{s}{2(1-2 \epsilon)} \operatorname{Tri}^{(4)}(s)-t \operatorname{Tri}^{(4)}(t)-\frac{1}{2}(s+2(1-2 \epsilon) t) \operatorname{Box}^{(6)}(s, t), \\
A_{q^{+} \bar{q}^{-} g^{+} g^{-}}^{R}= & -\frac{\epsilon\left(1-\epsilon \delta_{R}\right) s}{4 u}\left[\frac{1}{(1-\epsilon)(1-2 \epsilon)}((1-\epsilon) s+(1-2 \epsilon) t) \operatorname{Tri}^{(4)}(s)-\right. \\
& \left.\left.-\epsilon t \operatorname{Tri}^{(4)}(t)\right)+(s+2 t) \operatorname{Box}^{(6)}(s, t)\right]- \\
& -\frac{s}{2(1-2 \epsilon)} \operatorname{Tri}^{(4)}(s)-\frac{s}{2} \operatorname{Box}^{(6)}(s, t), \\
A_{q^{+} \bar{q}^{-} g^{+} g^{-}}^{L,[/ 2]} & 0, \\
A_{q^{+} g^{+} \bar{q}^{-} g^{+}}^{R}= & \frac{1}{4} \epsilon t\left(1-\epsilon \delta_{R}\right)\left[\frac{\epsilon}{(1-\epsilon)(1-2 \epsilon)}\left(\operatorname{Tri}^{(4)}(s)+\operatorname{Tri}^{(4)}(t)\right)-\operatorname{Box}^{(6)}(s, t)\right], \\
A_{q^{+} g^{-} \bar{q}^{-} g^{+}}^{R}= & \frac{1}{4} \epsilon\left(1-\epsilon \delta_{R}\right) t\left[\frac{1}{1-2 \epsilon} \operatorname{Tri}^{(4)}(t)+\operatorname{Box}^{(6)}(s, t)\right]-\frac{s}{2} \operatorname{Tri}^{(4)}(s)- \\
& -\frac{t}{2(1-2 \epsilon)} \operatorname{Tri}^{(4)}(t)-\frac{1}{2}((1-2 \epsilon) s+t) \operatorname{Box}^{(6)}(s, t) .
\end{aligned}
$$

In assigning helicity labels to the above primitive amplitudes we take the quark legs to be incoming.

Here $\operatorname{Tri}^{(4)}(s)$ is the scalar triangle integral in $4-2 \epsilon$ dimensions with one external massive leg, and $\operatorname{Box}^{(6)}(s, t)$ is the all-massless scalar box integral in $6-2 \epsilon$ dimensions. The explicit value of the triangle integral is

$$
\operatorname{Tri}^{(4)}(s)=-\frac{r_{\Gamma}}{\epsilon^{2}}(-s)^{-1-\epsilon},
$$

where

$$
\begin{aligned}
r_{\Gamma} & =e^{-\epsilon \psi(1)} \frac{\Gamma(1+\epsilon) \Gamma^{2}(1-\epsilon)}{\Gamma(1-2 \epsilon)} \\
& =1-\frac{1}{2} \zeta_{2} \epsilon^{2}-\frac{7}{3} \zeta_{3} \epsilon^{3}-\frac{47}{16} \zeta_{4} \epsilon^{4}+\mathcal{O}\left(\epsilon^{5}\right),
\end{aligned}
$$

with

$$
\zeta_{s} \equiv \sum_{n=1}^{\infty} n^{-s}, \quad \zeta_{2}=\frac{\pi^{2}}{6}, \quad \zeta_{3}=1.202057 \ldots, \quad \zeta_{4}=\frac{\pi^{4}}{90} .
$$

In the $s$-channel $(s>0)$, the $\epsilon$-expansion of eq. (3.22) is given by using the analytic continuation $\ln (-s) \rightarrow \ln s-i \pi$. The $D=6-2 \epsilon$ scalar box integral is completely finite as $\epsilon \rightarrow 0$. Its expansions to $\mathcal{O}\left(\epsilon^{2}\right)$ in the various kinematic channels are given, for example, in refs. $[3,9]$.

\subsection{Finite remainders}

Next we tabulate the finite remainders of the one-loop $q \bar{q} \rightarrow g g$ and $q g \rightarrow g q$ amplitudes at

$\mathcal{O}\left(\epsilon^{0}\right)$, defined by $\mathcal{M}_{q \bar{q} \rightarrow g g}^{(1) \mathrm{fin}}$ and $\mathcal{M}_{q g \rightarrow g q}^{(1) \text { fin }}$ in eq. (2.10) and color decomposed into $M_{h}^{(1),[c] \mathrm{fin}}$ 
in eq. (2.29). We write,

$$
\begin{aligned}
& M_{h}^{(1),[c] \text { fin }}=\left[-b_{0}(\right.\left.\left.\ln \left(s / \mu^{2}\right)-i \pi\right)+\frac{C_{F}}{2}\left(1-\delta_{R}\right)\right] M_{h}^{(0),[c]} \\
&+N a_{h}^{[c]}+\frac{1}{N} b_{h}^{[c]}+N_{f} d_{h}^{[c]}, \quad c=1,2, \\
& M_{h}^{(1),[c] \mathrm{fin}}=h_{h}^{[c]}+\frac{N_{f}}{N} j_{h}^{[c]}, \quad c=3 .
\end{aligned}
$$

For helicity configuration $h=1$, Bose symmetry under exchange of legs 3 and $4(t \leftrightarrow u)$ implies (see eq. (3.6)) that

$$
\begin{aligned}
a_{1}^{[2]}(s, t, u) & =-\frac{t}{u} a_{1}^{[1]}(s, u, t), \\
b_{1}^{[2]}(s, t, u) & =-\frac{t}{u} b_{1}^{[1]}(s, u, t), \\
d_{1}^{[2]}(s, t, u) & =-\frac{t}{u} d_{1}^{[1]}(s, u, t) .
\end{aligned}
$$

For the $h=1$ amplitude, the independent remainder functions $a, b, d, h$ and $j$ are

$$
\begin{aligned}
a_{1}^{[1]} & =-\frac{x}{6}-\frac{1}{4}, \\
b_{1}^{[1]} & =-\frac{1}{4}, \\
d_{1}^{[1]} & =\frac{x}{6} \\
h_{1}^{[3]} & =0 \\
j_{1}^{[3]} & =0
\end{aligned}
$$

where

$$
x=\frac{t}{s}, \quad y=\frac{u}{s}, \quad X=\ln \left(-\frac{t}{s}\right), \quad Y=\ln \left(-\frac{u}{s}\right) .
$$

For $h=2$ the functions are

$$
\begin{aligned}
a_{2}^{[1]}= & -\frac{(x-y)(1-x y)}{4 y^{3}} X^{2}-\frac{6 x^{2}-3 x y+11 y^{2}}{12 y^{2}} X+\frac{1}{4 y}-\frac{3}{2}+ \\
& +i \pi\left[-\frac{(x-y)(1-x y)}{2 y^{3}} X-\frac{6 x^{2}-3 x y+11 y^{2}}{12 y^{2}}\right] \\
b_{2}^{[1]}= & \frac{X^{2}}{4 y^{3}}-\frac{2-y}{4 y^{2}} X+\frac{1}{4 y}+2+i \pi\left[\frac{X}{2 y^{3}}-\frac{2-y}{4 y^{2}}\right] \\
d_{2}^{[1]}= & \frac{X}{6}+\frac{i \pi}{6}, \\
a_{2}^{[2]}= & \frac{x-y}{4 y} Y^{2}-\frac{5}{3} \frac{x}{y} Y-\frac{3}{2} \frac{x}{y}+i \pi\left[\frac{x-y}{2 y} Y-\frac{5}{3} \frac{x}{y}\right] \\
b_{2}^{[2]}= & \frac{Y^{2}}{4 y}+2 \frac{x}{y}+i \pi \frac{Y}{2 y}, \\
d_{2}^{[2]}= & \frac{x}{6 y} Y+i \pi \frac{x}{6 y},
\end{aligned}
$$




$$
\begin{aligned}
h_{2}^{[3]}= & -\frac{x(1-2 x)}{4 y^{2}} X^{2}-\frac{X Y}{y}-\frac{Y^{2}}{2}+\frac{5}{3} \frac{x}{y} X+\frac{5}{3} Y+\frac{\pi^{2}}{2 y}+ \\
& +i \pi\left[\frac{2-x y+x^{2}}{2 y^{2}} X+\frac{x}{y} Y-\frac{5}{3 y}\right], \\
j_{2}^{[3]}= & -\frac{x}{6 y} X-\frac{Y}{6}+\frac{i \pi}{6 y} .
\end{aligned}
$$

For $h=3$ the functions are

$$
\begin{aligned}
& a_{3}^{[1]}=-\frac{1}{6 x}-\frac{1}{4}, \\
& b_{3}^{[1]}=-\frac{1}{4}, \\
& d_{3}^{[1]}=\frac{1}{6 x}, \\
& a_{3}^{[2]}=\frac{1}{6 x}+\frac{1}{4 y}, \\
& b_{3}^{[2]}=\frac{1}{4 y}, \\
& d_{3}^{[2]}=-\frac{1}{6 x}, \\
& h_{3}^{[3]}=0, \\
& j_{3}^{[3]}=0 .
\end{aligned}
$$

For $h=4$ the functions are

$$
\begin{aligned}
a_{4}^{[1]}= & -\frac{(1-y)(1-x y)}{4 y^{3}} X^{2}+\frac{6 x^{2}+15 x y-2 y^{2}}{12 y^{2}} X+\frac{x}{4 y}-\frac{3}{2}+ \\
& +i \pi\left[-\frac{(1-y)(1-x y)}{2 y^{3}} X+\frac{6 x^{2}+15 x y-2 y^{2}}{12 y^{2}}\right], \\
b_{4}^{[1]}= & \frac{x^{3}}{4 y^{3}} X^{2}+\frac{x(2 x-y)}{4 y^{2}} X+\frac{x}{4 y}+2+i \pi\left[\frac{x^{3}}{2 y^{3}} X+\frac{x(2 x-y)}{4 y^{2}}\right], \\
d_{4}^{[1]}= & \frac{X}{6}+\frac{i \pi}{6}, \\
a_{4}^{[2]}= & \frac{1-y}{4 y}\left((X-Y)^{2}+\pi^{2}\right)-\frac{X+10 Y}{6 y}-\frac{3}{2 y}-i \pi \frac{11}{6 y}, \\
b_{4}^{[2]}= & \frac{x}{4 y}\left((X-Y)^{2}+\pi^{2}\right)+\frac{2}{y}, \\
d_{4}^{[2]}= & \frac{X+Y}{6 y}+\frac{i \pi}{3 y}, \\
h_{4}^{[3]}= & -\frac{x(1-2 x)}{4 y^{2}} X^{2}-\frac{Y^{2}}{2}-\frac{X Y}{y}+\frac{\pi^{2}}{2 y}+\frac{5}{3} \frac{x}{y} X+\frac{5}{3} Y+ \\
& +i \pi\left[\left(-\frac{x(1-2 x)}{2 y^{2}}-\frac{1}{y}\right) X+\frac{x}{y} Y-\frac{5}{3 y}\right] \\
j_{4}^{[3]}= & -\frac{x}{6 y} X-\frac{1}{6} Y+\frac{i \pi}{6 y} .
\end{aligned}
$$


For $h=5$ the functions are

$$
\begin{aligned}
a_{5}^{[1]}= & -\frac{1-y}{4 y} X^{2}-\frac{X}{6}-\frac{3}{2}+i \pi\left[-\frac{1-y}{2 y} X-\frac{1}{6}\right] \\
b_{5}^{[1]}= & \frac{x}{4 y} X^{2}+2+i \pi \frac{x}{2 y} X \\
d_{5}^{[1]}= & \frac{X}{6}+\frac{i \pi}{6} \\
a_{5}^{[2]}= & \frac{(1-y)(1-x y)}{4 y}\left((X-Y)^{2}+\pi^{2}\right)-\frac{11 x^{2}+19 x y+2 y^{2}}{12 y}(X-Y)-\frac{11}{6 y} Y- \\
& -\frac{7}{4 y}-\frac{1}{4}-i \pi \frac{11}{6 y}, \\
b_{5}^{[2]}= & \frac{x^{3}}{4 y}\left((X-Y)^{2}+\pi^{2}\right)-\frac{x(1-2 x)}{4 y}(X-Y)+\frac{7}{4 y}-\frac{1}{4} \\
d_{5}^{[2]}= & \frac{X+Y}{6 y}+\frac{i \pi}{3 y}, \\
h_{5}^{[3]}= & \frac{2-3 x y}{4 y}\left((X-Y)^{2}+\pi^{2}\right)+\frac{1}{2} X^{2}+\frac{x}{2 y} Y^{2}+\frac{5}{3} \frac{x}{y} X+\frac{5}{3} Y+ \\
& +i \pi\left[X+\frac{x}{y} Y-\frac{5}{3 y}\right] \\
j_{5}^{[3]}= & -\frac{x}{6 y} X-\frac{1}{6} Y+\frac{i \pi}{6 y} .
\end{aligned}
$$

For the HV scheme $\left(\delta_{R}=1\right)$, the results $(3.25)-(3.67)$ for the finite remainders of the one-loop helicity amplitudes are in complete agreement with those of ref. [42].

\subsection{Comparison with CDR results}

Results in the CDR scheme are usually phrased in terms of amplitude interferences, summed over all polarizations and colors. In the NNLO cross section, the one-loop amplitude enters interfered with itself. The contribution of the one-loop finite remainders to the NNLO $q \bar{q} \rightarrow g g$ or $q g \rightarrow g q$ cross section, summed over all helicities and colors, is given by

$$
\bar{I}^{(1,1) \mathrm{fin}} \equiv \sum_{\lambda_{i}= \pm 1}\left\langle\mathcal{M}_{\lambda_{1} \lambda_{2} \lambda_{3} \lambda_{4}}^{(1) \mathrm{fin}} \mid \mathcal{M}_{\lambda_{1} \lambda_{2} \lambda_{3} \lambda_{4}}^{(1) \mathrm{fin}}\right\rangle
$$

Using the color sum matrix $\mathcal{C C}_{i j}$ in eq. (2.38), and table 1 to relate $\lambda_{1} \lambda_{2} \lambda_{3} \lambda_{4}$ to $h$ helicity configurations, the color and helicity sum in $\bar{I}^{(1,1) \mathrm{fin}}$ may be evaluated in terms of the above explicit expressions (3.25)-(3.67) for $M_{h}^{(1),[c] \text { fin }}$, in a general $\delta_{R}$ scheme.

In ref. [5], the one-loop-squared contributions to the $q \bar{q} \rightarrow g g$ and $q g \rightarrow q g$ cross sections were given in the CDR scheme, but not in a convenient form for comparing to our results, because the infrared poles were not organized in the same way. (Also, the terms related to ultraviolet renormalization in eq. (4.1) of the original version of ref. [5] require correction.) However, the authors of ref. [5] have kindly supplied us with their versions of the values of eq. (3.68) in both the HV and CDR schemes. These expressions agree precisely with our result for eq. (3.68) in the HV scheme $\left(\delta_{R}=1\right)$. 


\section{Two-loop QCD amplitudes and finite remainders}

We generated the Feynman graphs for $q \bar{q} \rightarrow g g$ using QGRAF [54], from which a MAPLE program was constructed to evaluate each graph. As a cross-check, some of the diagrams were evaluated using FORM [55]. We employed the general integral reduction algorithms developed for the all-massless four-point topologies $[17,18,16,15]$, in order to reduce the loop integrals to a minimal basis of master integrals. To put the integrands into a form suitable for applying the general reduction algorithms, spinor strings were converted to traces over $\gamma$ matrices, by multiplying and dividing by appropriate spinor inner products constructed from the external momenta. Evaluating the traces then gave dot products of momenta; any terms containing an odd number of Levi-Civita tensors $\varepsilon^{\mu \nu \sigma \rho}$ vanished upon integration. The gluon polarization vectors of definite helicity can be incorporated using some minor extensions of the integral reduction techniques [9]. Here we incorporated the gluon polarization vectors in a slightly differently fashion than described there. When forming the traces over $\gamma$ matrices we included also the polarization vectors expressed in terms of spinors [32], following the methods used in, for example, refs. [35, 56]. Because the polarization vectors are four-dimensional objects, they distinguish between 4-dimensional and $(-2 \epsilon)$-dimensional components of the loop momentum. We evaluated the resulting integrals, containing $(-2 \epsilon)$-dimensional components of the loop momentum, using the methods of ref. [9]. (See refs. [48, 11, 42] for alternative multi-loop helicity techniques.)

After all the tensor loop integrals in the amplitudes have been reduced to a linear combination of master integrals, the next step is to expand the master integrals in a Laurent series in $\epsilon$, beginning at order $1 / \epsilon^{4}$, using results from refs. [13, 14, 17, 16, 18]. It is straightforward [57] to express the results solely in terms of polylogarithms [58],

$$
\begin{aligned}
\operatorname{Li}_{n}(x) & =\sum_{i=1}^{\infty} \frac{x^{i}}{i^{n}}=\int_{0}^{x} \frac{d t}{t} \operatorname{Li}_{n-1}(t), \\
\operatorname{Li}_{2}(x) & =-\int_{0}^{x} \frac{d t}{t} \ln (1-t),
\end{aligned}
$$

with $n=2,3,4$. The analytic properties of the non-planar double box integrals appearing in the amplitudes are somewhat intricate $[2,14]$; there is no Euclidean region in any of the three kinematic channels, $s, t$ or $u$. So we do not attempt to give a crossing-symmetric representation, but instead quote all our results in the physical $s$-channel $(s>0 ; t, u<0)$ for both the $q \bar{q} \rightarrow g g$ and $q g \rightarrow g q$ kinematics, eqs. (2.1) and (2.2).

\subsection{Finite remainders}

The two-loop finite remainders are defined in eq. (2.11) and are color decomposed into $M_{h}^{(2),[c] \text { fin }}$ in eq. (2.29). Their dependence on the renormalization scale $\mu$, color factors $N$ and $N_{f}$, and scheme label $\delta_{R}$ may be extracted as

$$
\begin{array}{r}
M_{h}^{(2),[c] \text { fin }}=\left[-b_{0}^{2}\left(\ln \left(s / \mu^{2}\right)-i \pi\right)^{2}-b_{1}\left(\ln \left(s / \mu^{2}\right)-i \pi\right)+\left(-\frac{1}{6} C_{A}+\frac{1}{8} C_{F}\right) C_{F}\left(1-\delta_{R}\right)^{2}+\right. \\
\left.+\left(2 R_{q}+2 R_{g}+b_{0} Q_{1}^{(q g)}\left(\ln \left(s / \mu^{2}\right)-i \pi\right)+\frac{1}{2} C_{F} Q_{0} i \pi\right)\left(1-\delta_{R}\right)\right] M_{h}^{(0),[c]}+
\end{array}
$$




$$
\begin{aligned}
& +\left[-2 b_{0}\left(\ln \left(s / \mu^{2}\right)-i \pi\right)+Q_{1}^{(q g)}\left(1-\delta_{R}\right)\right] M_{h}^{(1),[c] \mathrm{fin}}+ \\
& +Q_{0}\left[M_{h}^{(1),[c] \epsilon, \delta_{R}}-C_{A} V_{h}^{(1),[c]}\right]\left(1-\delta_{R}\right)+ \\
& +N^{2} A_{h}^{[c]}+B_{h}^{[c]}+\frac{1}{N^{2}} C_{h}^{[c]}+N N_{f} D_{h}^{[c]}+\frac{N_{f}}{N} E_{h}^{[c]}+ \\
& +N_{f}^{2} F_{h}^{[c]}+\frac{N_{f}^{2}}{N^{2}} G_{h}^{[c]}, \quad c=1,2, \\
M_{h}^{(2),[c] f i n}= & \left.-2 b_{0}\left(\ln \left(s / \mu^{2}\right)-i \pi\right)+Q_{1}^{(q g)}\left(1-\delta_{R}\right)\right] M_{h}^{(1),[c] \mathrm{fin}}+ \\
& +Q_{0}\left[M_{h}^{(1),[c] \epsilon, \delta_{R}}-C_{A} V_{h}^{(1),[c]}\right]\left(1-\delta_{R}\right)+ \\
& +N H_{h}^{[c]}+\frac{1}{N} I_{h}^{[c]}+N_{f} J_{h}^{[c]}+\frac{N_{f}}{N^{2}} K_{h}^{[c]}+\frac{N_{f}^{2}}{N} L_{h}^{[c]}, \quad c=3 .
\end{aligned}
$$

The $\mu$-dependence is a consequence of renormalization group invariance.

The tree and one-loop functions, $M_{h}^{(0),[c]}$ and $M_{h}^{(1),[c] f \text { in }}$, are given in eq. (2.36) and eqs. (3.25)-(3.67), respectively, while $b_{0}$ and $b_{1}$ are given in eq. (2.6). The following combinations of color constants also appear in eqs. (4.3) and (4.4),

$$
\begin{aligned}
Q_{0} & =\frac{5}{6} C_{A}-C_{F}+\frac{1}{3} T_{R} N_{f}, \\
Q_{1}^{(q g)} & =-\frac{1}{6} C_{A}+\frac{1}{2} C_{F}, \\
R_{q} & =-\frac{7}{48} C_{A}^{2}+\left(\frac{\pi^{2}}{192}+\frac{617}{864}\right) C_{A} C_{F}-\left(\frac{\pi^{2}}{24}+\frac{1}{4}\right) C_{F}^{2}-\frac{1}{16} C_{F} T_{R} N_{f}, \\
R_{g} & =\left(-\frac{5}{576} \pi^{2}+\frac{5}{48}\right) C_{A}^{2}+\left(\frac{2}{27} C_{A}-\frac{1}{8} C_{F}\right) T_{R} N_{f},
\end{aligned}
$$

where the combination $4 R_{g}$ appears as well in the $\delta_{R}$ dependence of the $g g \rightarrow g g$ amplitude, eq. (5.10) of ref. [9]. The quantities $M_{h}^{(1),[c] \epsilon, \delta_{R}}$ are the $\delta_{R^{-}}$dependent parts of the $\mathcal{O}\left(\epsilon^{1}\right)$ coefficients of the one-loop amplitude remainders, after subtracting the poles according to eq. (2.10). The explicit values of $M_{h}^{(1),[c] \epsilon, \delta_{R}}$ are tabulated in appendix B. Finally, the quantities $V_{h}^{(1),[c]}$ are only non-vanishing in the case of the simpler helicity configurations, $h=1$ and $h=3$. They seem to be related to the $\delta_{R}$ dependence of one-loop splitting amplitudes [59]. Their explicit values are given by

$$
\begin{array}{lll}
V_{1}^{(1),[1]}=\frac{x}{6}, & V_{1}^{(1),[2]}=-\frac{x}{6}, & V_{1}^{(1),[3]}=0, \\
V_{3}^{(1),[1]}=\frac{1}{6 x}, & V_{3}^{(1),[2]}=-\frac{1}{6 x}, & V_{3}^{(1),[3]}=0 .
\end{array}
$$

The coefficient functions $A, B, C, D, E, F, G, H, I, J, K, L$ depend only on the Mandelstam variables. In appendix $A$, we give the explicit forms for the independent finite remainder functions appearing in eqs. (4.3) and (4.4).

We have compared our results for the independent two-loop finite remainder functions $M_{h}^{(2),[c] \text { fin }}$ with corresponding results obtained contemporaneously in ref. [42]. The results agree completely, once a correction is made for a slightly different definition of $\boldsymbol{H}^{(2)}(\epsilon)$ in eq. (2.18). (We always dress the $1 / \epsilon$ pole with $\left(\mu^{2} /(-s)\right)^{2 \epsilon}$; ref. [42] sometimes dresses it with $\left(\mu^{2} /(-t)\right)^{2 \epsilon}$ or $\left(\mu^{2} /(-u)\right)^{2 \epsilon}$.) 


\subsection{Comparison with CDR results}

Finally we discuss conversion from the HV scheme results reported in section 4 to the CDR scheme used in ref. [5]. In the CDR scheme, one usually computes the interference of amplitudes, summed over all external colors and $(2-2 \epsilon)$ polarizations. The generic one-loop/tree interference encountered at NLO is

$$
2 \operatorname{Re} \bar{I}_{\text {R.S. }}^{(1,0)} \equiv 2 \operatorname{Re} \sum_{\text {color,hel. }}\left[\left\langle\mathcal{M}_{n}^{(1)} \mid \mathcal{M}_{n}^{(0)}\right\rangle\right]_{\text {R.S. }}
$$

Inserting the infrared decomposition (2.10) for $\mathcal{M}_{n}^{(1)}$ into eq. (4.11) gives

$$
\bar{I}_{\mathrm{RSS}}^{(1,0)}=2 \operatorname{Re} \sum_{\text {color,hel. }}\left[\left\langle\mathcal{M}_{n}^{(0)}\left|\boldsymbol{I}^{(1)}\right| \mathcal{M}_{n}^{(0)}\right\rangle\right]_{\mathrm{RSS}}+\bar{I}_{\mathrm{RS}}^{(1,0) \text { fin }}
$$

where

$$
\bar{I}_{\mathrm{RS} \text {. }}^{(1,0) \mathrm{fin}}=2 \operatorname{Re} \sum_{\text {color,hel. }}\left[\left\langle\mathcal{M}_{n}^{(1) \mathrm{fin}} \mid \mathcal{M}_{n}^{(0)}\right\rangle\right]_{\mathrm{R.S}} .
$$

It is well-established from explicit calculations and general arguments $[46,50,51]$ that the finite remainder (4.13) has the same value in the HV and CDR schemes, in the limit $\epsilon \rightarrow 0$. Essentially, the treatment of unobserved partons is the same in both schemes, so the infrared divergences should take the same form, when expressed in terms of the lower-order-in- $\alpha_{s}$ amplitudes.

It is natural to expect the same pattern to hold at two loops. (Indeed it does for the $g g \rightarrow g g$ amplitude $[6,9]$.) The two-loop/tree interference is

$$
\begin{aligned}
2 \operatorname{Re} \bar{I}_{\mathrm{RSS}}^{(2,0)} & \equiv 2 \operatorname{Re} \sum_{\text {color,hel. }}\left[\left\langle\mathcal{M}_{n}^{(2)} \mid \mathcal{M}_{n}^{(0)}\right\rangle\right]_{\mathrm{R.S} .} \\
& =2 \operatorname{Re} \sum_{\text {color,hel. }}\left[\left\langle\mathcal{M}_{n}^{(0)}\left|\boldsymbol{I}^{(2)}\right| \mathcal{M}_{n}^{(0)}\right\rangle+\left\langle\mathcal{M}_{n}^{(1)}\left|\boldsymbol{I}^{(1) \dagger}\right| \mathcal{M}_{n}^{(0)}\right\rangle\right]_{\mathrm{R} . \mathrm{S} .}+\bar{I}_{\mathrm{R} . \mathrm{S} .}^{(2,0) \mathrm{fin}},
\end{aligned}
$$

where

$$
\bar{I}_{\mathrm{RSS}}^{(2,0) \mathrm{fin}}=2 \operatorname{Re} \sum_{\text {color,hel. }}\left[\left\langle\mathcal{M}_{n}^{(2) \mathrm{fin}} \mid \mathcal{M}_{n}^{(0)}\right\rangle\right]_{\mathrm{R.S} .} .
$$

Note that $\boldsymbol{I}^{(1)}$ and $\boldsymbol{I}^{(2)}$ are the same operators in the HV scheme as in the CDR scheme.

We have interfered the color-decomposed finite remainders of the two-loop $q \bar{q} \rightarrow g g$ and $q g \rightarrow g q$ helicity amplitudes in the HV scheme, as given in section 4.1, with the tree amplitudes given in eq. (2.36), summing over all external helicities and colors with the help of eq. (2.38). This sum gives precisely the same result as the corresponding quantity (4.16) in the CDR scheme, as evaluated in ref. [5], after accounting for the slightly different

definition of $\boldsymbol{H}^{(2)}$ that we used in eq. (2.18). This result provides additional evidence that eq. (4.16) should be the same in the HV or CDR schemes for general two-loop QCD scattering amplitudes. A similar conclusion has been reached independently [42]. 


\section{Amplitudes in pure $N=1$ super-Yang-Mills theory}

QCD amplitudes may be converted easily to amplitudes in $N=1$ pure super-Yang-Mills theory by modifying the fermions to be in the adjoint representation and by altering their multiplicity to be a single Majorana fermion. This theory is then supersymmetric with the fermion the gluino superpartner of the gluon. Besides the inherent interest in supersymmetric theories, a practical consequence is that supersymmetry imposes a set of powerful identities that provide non-trivial checks on the amplitudes, including their finite parts. The supersymmetry identities have been applied previously to the same one-loop amplitudes discussed here [50], but only through $\mathcal{O}\left(\epsilon^{0}\right)$, as needed in an NLO calculation. Here we extend the one-loop discussion to include all orders in the dimensional regularization parameter $\epsilon$, which are relevant at two loops via Catani's formula (2.11). Then we verify the identities at two loops.

\subsection{Supersymmetry Ward Identities}

One set of identities implies that "maximal helicity violating" amplitudes vanish for any supersymmetric theory and any number of loops,

$$
\begin{aligned}
& \mathcal{M}^{\mathrm{SUSY}}\left(g_{1}^{ \pm}, g_{2}^{-}, g_{3}^{+}, \ldots, g_{n}^{+}\right)=0, \\
& \mathcal{M}^{\mathrm{SUSY}}\left(\tilde{g}_{1}^{+}, \tilde{g}_{2}^{-}, g_{3}^{+}, \ldots, g_{n}^{+}\right)=0,
\end{aligned}
$$

where $g$ and $\tilde{g}$ denote a gluon and gluino, and the superscripts denote helicities. In this paper we use the convention that legs 1 and 2 are incoming and the remaining ones are outgoing. Other identities relate the non-vanishing supersymmetric helicity amplitudes for external gluons alone, to amplitudes where some of the gluons are replaced by gluinos. In particular, the two-gluino two-gluon amplitude can be expressed in terms of the four-gluon amplitude:

$$
\mathcal{M}^{\mathrm{SUSY}}\left(\tilde{g}_{1}^{+}, \tilde{g}_{2}^{-}, g_{3}^{-}, g_{4}^{+}\right)=\frac{\langle 23\rangle}{\langle 13\rangle} \mathcal{M}^{\mathrm{SUSY}}\left(g_{1}^{+}, g_{2}^{-}, g_{3}^{-}, g_{4}^{+}\right)
$$

This identity is somewhat more stringent than the ones in eqs. (5.1) and (5.2) because it relates distinct amplitudes containing the most intricate infrared divergences, up to order $1 / \epsilon^{4}$ poles at two loops. These relations are crossing symmetric, when a crossing symmetric definition [60] of the spinor products is used.

For the four-point case, the pure-gluon identities (5.1) have already been checked at one loop to all orders in $\epsilon$, and at two loops through $\mathcal{O}\left(\epsilon^{0}\right)$ in the FDH regularization scheme $[34,9]$.

\subsection{Color and infrared structure}

Since the gluinos are in the adjoint representation we use the same color basis as used for the four-gluon helicity amplitudes [9]

$$
\tilde{\mathcal{M}}_{h}^{(L)}=S_{h} \times \sum_{c=1}^{9} \operatorname{Tr}^{[c]} \times \tilde{M}_{h}^{(L),[c]},
$$


where

$$
\begin{aligned}
& \operatorname{Tr}^{[1]}=\operatorname{tr}\left(T^{a_{1}} T^{a_{2}} T^{a_{3}} T^{a_{4}}\right), \\
& \operatorname{Tr}^{[2]}=\operatorname{tr}\left(T^{a_{1}} T^{a_{2}} T^{a_{4}} T^{a_{3}}\right), \\
& \operatorname{Tr}^{[3]}=\operatorname{tr}\left(T^{a_{1}} T^{a_{4}} T^{a_{2}} T^{a_{3}}\right), \\
& \operatorname{Tr}^{[4]}=\operatorname{tr}\left(T^{a_{1}} T^{a_{3}} T^{a_{2}} T^{a_{4}}\right), \\
& \operatorname{Tr}^{[5]}=\operatorname{tr}\left(T^{a_{1}} T^{a_{3}} T^{a_{4}} T^{a_{2}}\right), \\
& \operatorname{Tr}^{[6]}=\operatorname{tr}\left(T^{a_{1}} T^{a_{4}} T^{a_{3}} T^{a_{2}}\right), \\
& \operatorname{Tr}^{[7]}=\operatorname{tr}\left(T^{a_{1}} T^{a_{2}}\right) \operatorname{tr}\left(T^{a_{3}} T^{a_{4}}\right), \\
& \operatorname{Tr}^{[8]}=\operatorname{tr}\left(T^{a_{1}} T^{a_{3}}\right) \operatorname{tr}\left(T^{a_{2}} T^{a_{4}}\right), \\
& \operatorname{Tr}^{[9]}=\operatorname{tr}\left(T^{a_{1}} T^{a_{4}}\right) \operatorname{tr}\left(T^{a_{2}} T^{a_{3}}\right) .
\end{aligned}
$$

A reflection identity implies that the $c=4,5,6$ coefficients are equal to the $c=3,2,1$ coefficients (respectively), so there are really only six different coefficients for each $h$, namely $\tilde{M}_{h}^{(L),[c]}, c=1,2,3,7,8,9$.

Using $C, P$, and $T$, for the case of two external gluons and two external gluinos, there are five independent processes to consider, including processes related by crossing:

$$
\begin{array}{ll}
h=1: & \tilde{g}\left(p_{1},+\right)+\tilde{g}\left(p_{2},-\right) \rightarrow g\left(p_{3},+\right)+g\left(p_{4},+\right), \\
h=2: & \tilde{g}\left(p_{1},+\right)+\tilde{g}\left(p_{2},-\right) \rightarrow g\left(p_{3},-\right)+g\left(p_{4},+\right), \\
h=3: & \tilde{g}\left(p_{1},+\right)+g\left(p_{2},-\right) \rightarrow g\left(p_{3},+\right)+\tilde{g}\left(p_{4},+\right), \\
h=4: & \tilde{g}\left(p_{1},+\right)+g\left(p_{2},-\right) \rightarrow g\left(p_{3},-\right)+\tilde{g}\left(p_{4},+\right) . \\
h=5: & \tilde{g}\left(p_{1},+\right)+g\left(p_{2},+\right) \rightarrow g\left(p_{3},+\right)+\tilde{g}\left(p_{4},+\right) .
\end{array}
$$

The latter three processes are obtained from the first two by crossing. However, just as for the quark case we keep them distinct, because the crossing properties at two loops are in principle nontrivial.

The infrared divergence structure is similar to that of gluon-gluon scattering amplitudes [9]. For the case of $N=1$ pure super-Yang-Mills theory, in the basis (5.5) the matrix $\boldsymbol{I}^{(1)}$ is $[6,9]$

$$
\begin{gathered}
\tilde{\boldsymbol{I}}^{(1)}(\epsilon)=-\frac{e^{-\epsilon \psi(1)}}{\Gamma(1-\epsilon)}\left(\frac{1}{\epsilon^{2}}+\frac{\tilde{b}_{0}}{N \epsilon}\right) \times \\
\times\left(\begin{array}{ccccccccc}
N(\mathrm{~S}+\mathrm{T}) & 0 & 0 & 0 & 0 & 0 & (\mathrm{~T}-\mathrm{U}) & 0 & (\mathrm{~S}-\mathrm{U}) \\
0 & N(\mathrm{~S}+\mathrm{U}) & 0 & 0 & 0 & 0 & (\mathrm{U}-\mathrm{T}) & (\mathrm{S}-\mathrm{T}) & 0 \\
0 & 0 & N(\mathrm{~T}+\mathrm{U}) & 0 & 0 & 0 & 0 & (\mathrm{~T}-\mathrm{S}) & (\mathrm{U}-\mathrm{S}) \\
0 & 0 & 0 & N(\mathrm{~T}+\mathrm{U}) & 0 & 0 & 0 & (\mathrm{~T}-\mathrm{S}) & (\mathrm{U}-\mathrm{S}) \\
0 & 0 & 0 & 0 & N(\mathrm{~S}+\mathrm{U}) & 0 & (\mathrm{U}-\mathrm{T}) & (\mathrm{S}-\mathrm{T}) & 0 \\
0 & 0 & 0 & 0 & 0 & N(\mathrm{~S}+\mathrm{T}) & (\mathrm{T}-\mathrm{U}) & 0 & (\mathrm{~S}-\mathrm{U}) \\
(\mathrm{S}-\mathrm{U}) & (\mathrm{S}-\mathrm{T}) & 0 & 0 & (\mathrm{~S}-\mathrm{T}) & (\mathrm{S}-\mathrm{U}) & 2 N \mathrm{~S} & 0 & 0 \\
0 & (\mathrm{U}-\mathrm{T}) & (\mathrm{U}-\mathrm{S}) & (\mathrm{U}-\mathrm{S}) & (\mathrm{U}-\mathrm{T}) & 0 & 0 & 2 N \mathrm{U} & 0 \\
(\mathrm{~T}-\mathrm{U}) & 0 & (\mathrm{~T}-\mathrm{S}) & (\mathrm{T}-\mathrm{S}) & 0 & (\mathrm{~T}-\mathrm{U}) & 0 & 0 & 2 N \mathrm{~T}
\end{array}\right)
\end{gathered}
$$


where $\mathrm{S}, \mathrm{T}$ and $\mathrm{U}$ are defined in eq. (2.33). For $N=1$ super-Yang-Mills theory the first two coefficients of the $\beta$-function are

$$
\tilde{b}_{0}=\frac{3}{2} C_{A}, \quad \tilde{b}_{1}=\frac{3}{2} C_{A}^{2} .
$$

The $\boldsymbol{I}^{(2)}$ operator for super-Yang-Mills theory is

$$
\begin{aligned}
\tilde{\boldsymbol{I}}_{\mathrm{FDH}}^{(2)}(\epsilon, \mu ;\{p\})= & -\frac{1}{2} \tilde{\boldsymbol{I}}^{(1)}(\epsilon, \mu ;\{p\})\left(\tilde{\boldsymbol{I}}^{(1)}(\epsilon, \mu ;\{p\})+\frac{2 \tilde{b}_{0}}{\epsilon}\right)+ \\
& +\frac{e^{+\epsilon \psi(1)} \Gamma(1-2 \epsilon)}{\Gamma(1-\epsilon)}\left(\frac{\tilde{b}_{0}}{\epsilon}+K_{\mathrm{FDH}}^{\mathrm{SYM}}\right) \tilde{\boldsymbol{I}}^{(1)}(2 \epsilon, \mu ;\{p\})+ \\
& +\tilde{\boldsymbol{H}}_{\mathrm{FDH}}^{(2)}(\epsilon, \mu ;\{p\}),
\end{aligned}
$$

where

$$
\begin{gathered}
K_{\mathrm{FDH}}^{\mathrm{SYM}}=\left(3-\frac{\pi^{2}}{6}-\frac{4}{9} \epsilon\right) C_{A}, \\
\tilde{\boldsymbol{H}}_{\mathrm{FDH}}^{(2)}(\epsilon, \mu ;\{p\})=\frac{e^{-\epsilon \psi(1)}}{4 \epsilon \Gamma(1-\epsilon)}\left(\frac{\mu^{2}}{-s}\right)^{2 \epsilon}\left(4\left(H_{g}^{(2)}\right)_{\mathrm{FDH}}^{\mathrm{SYM}} \mathbf{1}+\hat{\boldsymbol{H}}^{(2)}\right) .
\end{gathered}
$$

and

$$
\left(H_{g}^{(2)}\right)_{\mathrm{FDH}}^{\mathrm{SYM}}=\left(H_{\tilde{g}}^{(2)}\right)_{\mathrm{FDH}}^{\mathrm{SYM}}=\left(\frac{\zeta_{3}}{2}+\frac{\pi^{2}}{16}-\frac{2}{9}\right) C_{A}^{2} .
$$

The equality of $\left(H_{g}^{(2)}\right)_{\mathrm{FDH}}^{\mathrm{SYM}}$ and $\left(H_{\tilde{g}}^{(2)}\right)_{\mathrm{FDH}}^{\mathrm{SYM}}$ is a consequence of supersymmetry. Equations (5.14)-(5.16) are obtained from the QCD formulas, eqs. (2.17)-(2.20), by the replacements $\delta_{R} \rightarrow 0, C_{F} \rightarrow C_{A}$ and $T_{R} N_{F} \rightarrow C_{A} / 2$ for converting to a single adjoint fermion in the FDH scheme. The operator $\hat{\boldsymbol{H}}^{(2)}$ defined in eq. (2.21) does not explicitly depend on the fermion representation.

The tree amplitudes in this color basis are given by $\tilde{M}_{h}^{(0),[c]}$, where

$$
\begin{aligned}
\tilde{M}_{1}^{(0),[c]} & =\tilde{M}_{3}^{(0),[c]}=0, & c & =1,2, \ldots, 9, \\
\tilde{M}_{h}^{(0),[c]} & =0, & c & =7,8,9, \quad h=1,2,3,4,5, \\
\tilde{M}_{h}^{(0),[c]} & =1, & c & =1,6, \quad h=2,4,5, \\
\tilde{M}_{h}^{(0),[c]} & =\frac{t}{u}, & c & =2,4, \quad h=2,4,5, \\
\tilde{M}_{h}^{(0),[c]} & =\frac{s}{u}, & c & =3,4, \quad h=2,4,5 .
\end{aligned}
$$

\subsection{One-loop amplitudes in pure $N=1$ super-Yang-Mills theory}

We now present the results for one-loop two-gluino two-gluon scattering in a format valid to all orders in $\epsilon$. By comparing these results to the corresponding $N=1$ pure Yang-Mills four-gluon amplitudes [9], we then verify that the identities (5.2) and (5.3) do indeed hold at one loop to all orders in $\epsilon$ when the FDH scheme is used.

To check the supersymmetry Ward identities at one loop, we use a crossing symmetric representation of the amplitudes directly in terms of one-loop scalar integral functions. 
Thus it is sufficient to explicitly present only the $h=1,2$ helicity cases. Using formulae from ref. [31] for obtaining the $N=1$ supersymmetric amplitudes from the primitive amplitudes, the coefficients of the first color structure $\operatorname{Tr}^{[1]}$ in eq. (5.5) for $h=1,2$ are given by

$$
\begin{aligned}
\tilde{M}_{1}^{(1),[1]}(s, t, u)=N & \left(A^{L}\left(1_{q}^{+}, 2_{\bar{q}}^{-}, 3_{g}^{+}, 4_{g}^{+}\right)+A^{R}\left(1_{q}^{+}, 2_{\bar{q}}^{-}, 3_{g}^{+}, 4_{g}^{+}\right)+\right. \\
& \left.+A^{L,[1 / 2]}\left(1_{q}^{+}, 2_{\bar{q}}^{-}, 3_{g}^{+}, 4_{g}^{+}\right)\right) \\
\tilde{M}_{2}^{(1),[1]}(s, t, u)=N( & A^{L}\left(1_{q}^{+}, 2_{\bar{q}}^{-}, 3_{g}^{-}, 4_{g}^{+}\right)+A^{R}\left(1_{q}^{+}, 2_{\bar{q}}^{-}, 3_{g}^{-}, 4_{g}^{+}\right)+ \\
& \left.+A^{L,[1 / 2]}\left(1_{q}^{+}, 2_{\bar{q}}^{-}, 3_{g}^{-}, 4_{g}^{+}\right)\right)-\frac{\tilde{b}_{0}}{\epsilon} \tilde{M}_{2}^{(0),[1]}
\end{aligned}
$$

For the second color structure in eq. (5.5), we have

$$
\begin{aligned}
\tilde{M}_{1}^{(1),[2]}(s, t, u)=-N & \frac{t}{u}\left(A^{L}\left(1_{q}^{+}, 2_{\bar{q}}^{-}, 4_{g}^{+}, 3_{g}^{+}\right)+A^{R}\left(1_{q}^{+}, 2_{\bar{q}}^{-}, 4_{g}^{+}, 3_{g}^{+}\right)+\right. \\
& \left.+A^{L,[1 / 2]}\left(1_{q}^{+}, 2_{\bar{q}}^{-}, 4_{g}^{+}, 3_{g}^{+}\right)\right), \\
\tilde{M}_{2}^{(1),[2]}(s, t, u)=N \frac{t}{u} & \left(A^{L}\left(1_{q}^{+}, 2_{\bar{q}}^{-}, 4_{g}^{+}, 3_{g}^{-}\right)+A^{R}\left(1_{q}^{+}, 2_{\bar{q}}^{-}, 4_{g}^{+}, 3_{g}^{-}\right)+\right. \\
& \left.+A^{L,[1 / 2]}\left(1_{q}^{+}, 2_{\bar{q}}^{-}, 4_{g}^{+}, 3_{g}^{-}\right)\right)-\frac{\tilde{b}_{0}}{\epsilon} \tilde{M}_{2}^{(0),[2]} .
\end{aligned}
$$

The third color configuration $\operatorname{Tr}^{[3]}$ has coefficients given by,

$$
\begin{aligned}
& \tilde{M}_{1}^{(1),[3]}(s, t, u)=0 \\
& \tilde{M}_{2}^{(1),[3]}(s, t, u)=2 N \frac{s}{u} A^{R}\left(1_{q}^{+}, 3_{g}^{-}, 2_{\bar{q}}^{-}, 4_{g}^{+}\right)-\frac{\tilde{b}_{0}}{\epsilon} \tilde{M}_{2}^{(0),[3]} .
\end{aligned}
$$

In the above, $\tilde{b}_{0}$ is the first $\beta$-function coefficient for $N=1$ super-Yang-Mills theory, The remaining color coefficients are given in terms of these $[61,35]$,

$$
\begin{aligned}
\tilde{M}_{h}^{(1),[4]}(s, t, u) & =\tilde{M}_{h}^{(1),[3]}(s, t, u), \\
\tilde{M}_{h}^{(1),[5]}(s, t, u) & =\tilde{M}_{h}^{(1),[2]}(s, t, u) \\
\tilde{M}_{h}^{(1),[6]}(s, t, u) & =\tilde{M}_{h}^{(1),[1]}(s, t, u), \\
\tilde{M}_{h}^{(1),[7]}(s, t, u) & =\frac{2}{N}\left(\tilde{M}_{h}^{(1),[3]}(s, t, u)+\tilde{M}_{h}^{(1),[2]}(s, t, u)+\tilde{M}_{h}^{(1),[1]}(s, t, u)\right), \\
\tilde{M}_{h}^{(1),[8]}(s, t, u) & =\tilde{M}_{h}^{(1),[9]}(s, t, u)=\tilde{M}_{h}^{(1),[7]}(s, t, u),
\end{aligned}
$$

for all helicity configurations $h$.

Inserting the explicit values of the primitive amplitudes and taking the FDH scheme $\left(\delta_{R}=0\right)$, yields for the three independent color factors of the $h=1$ helicity configuration in eq. (5.6),

$$
\begin{aligned}
\tilde{M}_{1}^{(1),[1]}(s, t, u) & =0, \\
\tilde{M}_{1}^{(1),[2]}(s, t, u) & =0, \\
\tilde{M}_{1}^{(1),[3]}(s, t, u) & =0 .
\end{aligned}
$$


For the $h=2$ helicity configuration (5.7),

$$
\begin{aligned}
\tilde{M}_{2}^{(1),[1]}(s, t, u)=N[ & -\frac{s}{2 u} \frac{2 u+\epsilon(4 s+t)}{1-2 \epsilon} \operatorname{Tri}^{(4)}(s)-\frac{t}{2 u} \frac{2 u+\epsilon(4 t+s)}{1-2 \epsilon} \operatorname{Tri}^{(4)}(t)+ \\
& \left.+\frac{1}{2 u}\left[2 s^{2}+t s+2 t^{2}-\epsilon\left(4 s^{2}+5 t s+4 t^{2}\right)\right] \operatorname{Box}^{(6)}(s, t)\right]- \\
- & \frac{\tilde{b}_{0}}{\epsilon} \tilde{M}_{2}^{(0),[1]} \\
\tilde{M}_{2}^{(1),[2]}(s, t, u)=N[ & -\frac{2-\epsilon}{1-2 \epsilon} \frac{s t}{2 u} \operatorname{Tri}^{(4)}(s)-t \operatorname{Tri}^{(4)}(u)+ \\
& \left.+\frac{t(2 t+\epsilon(4 u+s))}{2 u} \operatorname{Box}^{(6)}(s, u)\right]-\frac{\tilde{b}_{0}}{\epsilon} \tilde{M}_{2}^{(0),[2]} \\
\tilde{M}_{2}^{(1),[3]}(s, t, u)=N[ & -\frac{2-\epsilon}{1-2 \epsilon} \frac{s t}{2 u} \operatorname{Tri}^{(4)}(t)-s \operatorname{Tri}^{(4)}(u)+ \\
& \left.+\frac{s(2 s+\epsilon(4 u+t))}{2 u} \operatorname{Box}^{(6)}(t, u)\right]-\frac{\tilde{b}_{0}}{\epsilon} \tilde{M}_{2}^{(0),[3]}
\end{aligned}
$$

For helicity $h=1$, the amplitudes clearly satisfy the supersymmetry Ward identity (5.2) exactly. To check identity (5.3), we compared the amplitudes in eqs. (5.28), (5.29) and (5.30) to the corresponding four-gluon amplitudes. The latter amplitudes may be obtained from section 3.1 of ref. [9], by letting $N_{f} \rightarrow N$ and $b_{0} \rightarrow \tilde{b}_{0}$ in eq. (3.1) of that paper. (Note that the " $N=1$ " there refers to a chiral multiplet consisting a scalar and a fermion, while here we are considering a pure super-Yang-Mills multiplet consisting of a gluon and a gluino.) The result of this comparison is that the supersymmetry identity (5.3) holds to all orders in $\epsilon$ when the FDH scheme is used.

We note that the symmetry relation evident between eqs. (5.29) and (5.30),

$$
\tilde{M}_{2}^{(1),[3]}(s, t, u)=\tilde{M}_{2}^{(1),[2]}(t, s, u),
$$

which involves a swap of a gluon and gluino leg, is a consequence of the Bose symmetry of the corresponding four-gluon amplitude, because the gluon legs being exchanged in the latter amplitude have the same helicity (when both are considered as outgoing states).

After performing the subtraction of infrared divergences using eqs. (2.10) and (5.11), we obtain

$$
\begin{aligned}
M_{h}^{(1), \mathrm{SYM},[c] \mathrm{fin}}=\left[-\tilde{b}_{0}\left(\ln \left(s / \mu^{2}\right)-i \pi\right)+\frac{1}{2} N\right] M_{h}^{(0),[c]}+N a_{h}^{\mathrm{SYM},[c]}, & c=1,2,3, \\
M_{h}^{(1), \mathrm{SYM},[c] \text { fin }}=g_{h}^{\mathrm{SYM},[c]}, & c=7,8,9,
\end{aligned}
$$

where the one-loop supersymmetric remainder functions are given in terms of the QCD ones,

$$
\begin{aligned}
a_{h}^{\mathrm{SYM},[c]} & =a_{h}^{[c]}-b_{h}^{[c]}+d_{h}^{[c]}, & c & =1,2,3, \\
g_{h}^{\mathrm{SYM},[c]} & =2\left(a_{h}^{\mathrm{SYM},[1]}+a_{h}^{\mathrm{SYM},[2]}+a_{h}^{\mathrm{SYM},[3]}\right), & c & =7,8,9 .
\end{aligned}
$$

The $N M_{h}^{(0),[c]} / 2$ term in eq. (5.32) is a consequence of the finite shift between the FDH scheme used in super-Yang-Mills theory and the HV scheme used in QCD. 


\subsection{Two-loop amplitudes in pure $N=1$ super-Yang-Mills theory}

An immediate consequence of the one-loop supersymmetry identities holding to all orders in $\epsilon$ is that all infrared divergent terms at two loops also satisfy the same identities. Since neither $\boldsymbol{I}^{(1)}$ in eq. (5.11) nor $\boldsymbol{I}^{(2)}$ in eq. (5.13) depend on whether the external lines are gluons or gluinos, the Catani formula (2.11) dictates that divergent terms at two loops must satisfy the same supersymmetry Ward identities as the tree and one-loop amplitudes do.

This then leaves the question of whether the two-loop finite terms satisfy supersymmetry Ward identities. For the case of four-gluon scattering, the finite remainder terms, after subtracting the Catani terms (including their finite parts), have been presented previously [9]. We have carried out the analogous subtraction using eqs. (2.11), (5.11) and (5.13). The result of this is,

$$
\begin{aligned}
M_{h}^{(2), \mathrm{SYM},[c] \mathrm{fin}}= & -\left[\left(\tilde{b}_{0}\right)^{2}\left(\ln \left(s / \mu^{2}\right)-i \pi\right)^{2}+\tilde{b}_{1}\left(\ln \left(s / \mu^{2}\right)-i \pi\right)\right] M_{h}^{(0),[c]}- \\
& -2 \tilde{b}_{0}\left(\ln \left(s / \mu^{2}\right)-i \pi\right) M_{h}^{(1), \mathrm{SYM},[c] \mathrm{fin}}+N^{2} A_{h}^{\mathrm{SYM},[c]}+B_{h}^{\mathrm{SYM},[c]}, \\
M_{h}^{(2), \mathrm{SYM},[c] \mathrm{fin}}= & -2 \tilde{b}_{0}\left(\ln \left(s / \mu^{2}\right)-i \pi\right) M_{h}^{(1), \mathrm{SYM},[c] \mathrm{fin}}+N G_{h}^{\mathrm{SYM},[c]}, \\
c=7,2,3, &
\end{aligned}
$$

where we have verified that the finite remainder functions match those of the pure gluon case, i.e.

$$
\begin{aligned}
X_{1}^{\mathrm{SYM},[c]} & =X_{3}^{\mathrm{SYM},[c]}=0, \\
X_{2}^{\mathrm{SYM},[c]} & =X_{4}^{\mathrm{SYM},[c]}=-\frac{s t}{u^{2}} X_{-+-+}^{\mathrm{SYM},[c]}, \\
X_{5}^{\mathrm{SYM},[c]} & =-\frac{t}{s} X_{--++}^{\mathrm{SYM},[c]}
\end{aligned}
$$

where $X \in\{A, B, G\}$. The functions $X_{-+-+}^{\mathrm{SYM},[c]}$ and $X_{--++}^{\mathrm{SYM},[c]}$ for $g g \rightarrow g g$ scattering in pure $N=1$ super-Yang-Mills theory are given in ref. [9]. (Note that in that reference an all outgoing definition of helicity is used.) The relations (5.38)-(5.40) are precisely equivalent to the content of the supersymmetry Ward identities [33], after removing overall factors and the divergent terms, and extracting the $N$ and $\mu$ dependence. Thus they provide a direct, nontrivial check on the finite remainders.

Because the adjoint color indices of the gluino fields are identical to those of gluons, and only structure constants $f^{a b c}$ appear in the two-loop Feynman diagrams, the color coefficients for two-gluino two-gluon scattering obey the same group theory relations identified for $g g \rightarrow g g$ in ref. [9],

$$
\begin{aligned}
& G_{h}^{\mathrm{SYM},[7]}=2\left(A_{h}^{\mathrm{SYM},[1]}+A_{h}^{\mathrm{SYM},[2]}+A_{h}^{\mathrm{SYM},[3]}\right)-B_{h}^{\mathrm{SYM},[3]} \\
& G_{h}^{\mathrm{SYM},[8]}=2\left(A_{h}^{\mathrm{SYM},[1]}+A_{h}^{\mathrm{SYM},[2]}+A_{h}^{\mathrm{SYM},[3]}\right)-B_{h}^{\mathrm{SYM},[1]} \\
& G_{h}^{\mathrm{SYM},[9]}=2\left(A_{h}^{\mathrm{SYM},[1]}+A_{h}^{\mathrm{SYM},[2]}+A_{h}^{\mathrm{SYM},[3]}\right)-B_{h}^{\mathrm{SYM},[2]}
\end{aligned}
$$


and

$$
B_{h}^{\mathrm{SYM},[3]}=-B_{h}^{\mathrm{SYM},[1]}-B_{h}^{\mathrm{SYM},[2]} .
$$

\section{Conclusions}

In this paper we have presented the two-loop amplitudes for quark-gluon scattering in QCD and gluino-gluon scattering in $N=1$ super-Yang-Mills theory, including the full dependence on external colors and helicities. We confirmed that, as in the case of gluongluon scattering, there is an additional $1 / \epsilon$ pole term, $\hat{\boldsymbol{H}}^{(2)}$ in eq. (2.21) having nontrivial color dependence. This additional term vanishes after interfering it with the tree amplitude and summing over colors. We investigated the dependence of the amplitudes on the variety of dimensional regularization employed. The FDH scheme respects the supersymmetry Ward identities, in what represents the most detailed test to date of these identities, and of the FDH scheme. The scheme dependence of the quark-gluon amplitudes is much more intricate than was the case for the four-gluon helicity amplitudes [9].

The two-loop QCD results, when interfered with the tree amplitude, summed over all external colors and helicities, and converted to the CDR scheme, are in complete agreement with the previous results of Anastasiou, Glover, Oleari, and Tejeda-Yeomans [5]. We also expressed the one-loop-squared contribution to the NNLO $q \bar{q} \rightarrow g g$ and $q g \rightarrow g q$ cross sections in terms of one-loop finite remainders. These also agree with suitably subtracted expressions in the CDR scheme [52].

So far the new two-loop amplitudes have been implemented in only a handful of phe-

nomenological studies $[8,62]$. We may anticipate that once general algorithms for dealing with infrared divergent phase space integrations at next-to-next-leading-order are developed (see e.g. ref. [63]), many more phenomenological applications will follow. These applications would include the implementation of the two-loop amplitudes of this paper, or those of ref. [5], as ingredients in a numerical program for computing dijet production cross sections at hadron colliders at NNLO in QCD. When this task is accomplished, the intrinsic precision on the QCD predictions should reach the few percent level, providing a stringent test of the Standard Model at short distances.

\section{Acknowledgments}

We thank Babis Anastasiou, David Kosower and Henry Wong for helpful comments. We also thank Babis Anastasiou, Nigel Glover and Maria Elena Tejeda-Yeomans for discussions regarding the one-loop-squared interference, and Nigel Glover and Maria Elena TejedaYeomans for communicating their results to us prior to publication. Z.B. thanks SLAC, and L.D. thanks UCLA, for hospitality while this paper was being completed.

\section{A. Finite remainder functions for QCD}

In this appendix, we present the explicit forms for the independent finite remainder functions for the processes $q \bar{q} \rightarrow g g$ and $q g \rightarrow g q$ in QCD, which appear in eqs. (4.3) and (4.4). 
For the helicity $h=1$ configuration in eq. (2.22) and color factor $\operatorname{Tr}^{[1]}$ in eq. (2.30), the finite remainder functions are:

$$
\begin{aligned}
& A_{1}^{[1]}=-\frac{1}{48 y^{2}}\left(30 x^{3}+51 x^{2} y+22 x y^{2}-3 y^{3}\right) X^{2}- \\
& -\frac{1}{144 y}\left(180 x^{2}+239 x y+99 y^{2}\right) X+\frac{25}{864} x+\frac{475}{288} y- \\
& -i \pi\left[\frac{1}{24 y^{2}}\left(30 x^{3}+51 x^{2} y+22 x y^{2}-3 y^{3}\right) X+\right. \\
& \left.+\frac{1}{144 y}\left(180 x^{2}+239 x y+99 y^{2}\right)\right] \text {, } \\
& B_{1}^{[1]}=\frac{x(y-4)}{16 y^{2}} X^{2}+\frac{x-y}{16 x} Y^{2}+\frac{x}{16}(1-6 y)\left((X-Y)^{2}+\pi^{2}\right)- \\
& -\frac{1}{8 y}\left(4 x^{2}+5 x y+7 y^{2}\right) X-\frac{3}{8}(x-y) Y+\frac{115}{72} x+\frac{139}{72} y+ \\
& +i \pi\left(-\frac{x(4-y)}{8 y^{2}} X+\frac{x-y}{8 x} Y-\frac{1}{2 y}\right), \\
& C_{1}^{[1]}=\frac{y^{2}-2 x^{2}}{16 y^{2}} X^{2}+\frac{x-y}{16 x} Y^{2}+\frac{x(1-6 y)}{16}\left((X-Y)^{2}+\pi^{2}\right)+ \\
& +\frac{1}{16 y}\left(4 x^{2}+13 x y-3 y^{2}\right) X-\frac{3}{8}(x-y) Y-\frac{17}{32}+ \\
& +i \pi\left(\frac{y^{2}-2 x^{2}}{8 y^{2}} X+\frac{x-y}{8 x} Y+\frac{3-x}{16 y}\right) \text {, } \\
& D_{1}^{[1]}=\frac{x}{24 y^{2}}\left(5-x y+x^{2}\right) X^{2}+\frac{1}{72 y}\left(9+5 x y+27 x^{2}\right) X+\frac{143}{108} x-\frac{37}{72} y+ \\
& +i \pi\left(\frac{x}{12 y^{2}}\left(5-x y+x^{2}\right) X+\frac{1}{72 y}\left(9+5 x y+27 x^{2}\right)\right) \text {, } \\
& E_{1}^{[1]}=\frac{x(2 x-1)}{8 y^{2}} X^{2}+\frac{1-2 y}{8 x} Y^{2}+\frac{x(x-y)}{8}\left((X-Y)^{2}+\pi^{2}\right)+ \\
& +\frac{1}{8 y}\left(y^{2}+7 x y-6 x^{2}\right) X-\frac{3}{4}(x-y) Y+\frac{7}{36} x-\frac{19}{72} y+ \\
& +i \pi\left(-\frac{x(1-2 x)}{4 y^{2}} X+\frac{1-2 y}{4 x} Y+\frac{6 x-7 y}{8 y}\right), \\
& F_{1}^{[1]}=\frac{x}{36} X-\frac{5}{54} x+\frac{i \pi}{36} x, \\
& G_{1}^{[1]}=0 \text {. }
\end{aligned}
$$

Bose symmetry under exchanges of legs 3 and $4(t \leftrightarrow u)$ implies that for $h=1$ the finite remainders of the second color configuration in eq. (2.30) can be expressed in terms of the first ones, i.e.

$$
Z_{1}^{[2]}(s, t, u)=-\frac{t}{u} Z_{1}^{[1]}(s, u, t),
$$

where $Z \in\{A, B, C, D, E, F, G\}$. 
For $h=1$ in eq. (2.23) and color factor $\operatorname{Tr}^{[3]}$ in eq. (2.30):

$$
\begin{aligned}
& H_{1}^{[3]}=\frac{1}{48 y^{2}}\left(-6-25 x+7 x^{2}-4 x^{3}\right) X^{2}-\frac{x}{18 y}(11+32 x) X- \\
& -\frac{1}{48 x y}\left(-6-25 y+7 y^{2}-4 y^{3}\right) Y^{2}+\frac{1}{18}(11+32 y) Y- \\
& -\frac{x-y}{8 y}(1-x y)\left((X-Y)^{2}+\pi^{2}\right)+ \\
& +i \pi\left[\frac{1}{24 y^{2}}\left(-6-25 x+7 x^{2}-4 x^{3}\right) X-\right. \\
& \left.-\frac{1}{24 x y}\left(-6-25 y+7 y^{2}-4 y^{3}\right) Y+\frac{7(x-y)}{6 y}\right] \\
& I_{1}^{[3]}=-\frac{1-x}{16 y} Y^{2}+\frac{1-y}{16 y} X^{2}-\frac{x-y}{8 y}(1+2 x y)\left((X-Y)^{2}+\pi^{2}\right)+ \\
& +\frac{1}{12}(1+12 x) Y-\frac{x}{12 y}(1+12 y) X+ \\
& +i \pi\left(\frac{1-y}{8 y} X-\frac{1-x}{8 y} Y-\frac{x-y}{12 y}\right) \\
& J_{1}^{[3]}=\frac{x\left(2 x^{2}+x+5\right)}{24 y^{2}} X^{2}-\frac{2 y^{2}+y+5}{24 x} Y^{2}+\frac{x}{72 y}(39 x-55 y) X- \\
& -\frac{1}{72}(39 y-55 x) Y-\frac{1}{4} x(x-y)\left((X-Y)^{2}+\pi^{2}\right)+ \\
& +i \pi\left(\frac{x}{12 y^{2}}\left(2 x^{2}+x+5\right) X-\frac{2 y^{2}+y+5}{12 x} Y-\frac{13(x-y)}{24 y}\right), \\
& K_{1}^{[3]}=\frac{x(1-2 x)}{8 y^{2}} X^{2}-\frac{1-2 y}{8 x} Y^{2}-\frac{1}{8} x(x-y)\left((X-Y)^{2}+\pi^{2}\right)+ \\
& +\frac{1}{24}(17 x-19 y) Y-\frac{x}{24 y}(17 y-19 x) X+ \\
& +i \pi\left(\frac{x(1-2 x)}{4 y^{2}} X-\frac{1-2 y}{4 x} Y-\frac{19(x-y)}{24 y}\right), \\
& L_{1}^{[3]}=\frac{1}{36} x(X-Y) \text {. }
\end{aligned}
$$

For $h=2$ in eq. (2.23) and color factor $\operatorname{Tr}^{[1]}$ in eq. (2.30):

$$
\begin{aligned}
A_{2}^{[1]}=\frac{1}{2 y^{3}}[ & \left(x^{3} \operatorname{Li}_{3}(-x)-\zeta_{3}\left(x^{3}+2 y^{3}\right)\right) X-\frac{1}{2}\left(3 x^{3}+y^{3}\right) \operatorname{Li}_{4}(-x)- \\
& -\frac{1}{4}(x-y)(1-x y) X^{2} \operatorname{Li}_{2}(-x)-\frac{1}{48}\left(7 x^{3}-3 y^{3}\right) X^{4}+ \\
& \left.\quad+\frac{\pi^{2}}{3}\left(2 x^{3}-y^{3}\right) X^{2}+\frac{\pi^{4}}{1440}\left(24 x^{3}+95 y^{3}\right)\right]- \\
- & \frac{1}{6} \pi^{2} \operatorname{Li}_{2}(-x)+\frac{1}{12} X Y\left(X^{2}-2 \pi^{2}\right)+ \\
+ & \frac{1}{144 y^{3}}\left[\left(-24 x^{2} y-77 y^{3}+21 x y^{2}+86 x^{3}\right) X^{3}+\right.
\end{aligned}
$$




$$
\begin{aligned}
& \left.+\left(576 y+401 y^{3}+684 y^{2}+172\right) \pi^{2} X\right]+ \\
& +\frac{1}{48 y^{2}}\left[\left(35 y^{2}+6 x^{2}-3 x y\right)\left(2 \operatorname{Li}_{2}(-x) X+X^{2} Y-2 \operatorname{Li}_{3}(-x)\right)+\right. \\
& \left.+\left(12 x y+241 y^{2}-24 x^{2}\right) \zeta_{3}\right]-\frac{1}{72 y^{2}}\left(45 y^{2}-49 x y+86 x^{2}\right) \pi^{2}- \\
& -\frac{1}{288 y^{3}}\left(99 x^{2} y-505 y^{3}+279 x y^{2}+475 x^{3}\right) X^{2}- \\
& -\frac{1}{864 y^{2}}\left(2850 x^{2}+609 x y-2273 y^{2}\right) X-\frac{475 x}{288 y}-\frac{36077}{3456}+ \\
& +i \pi\left[\frac{x^{3}}{2 y^{3}}\left(\operatorname{Li}_{3}(-x)-\zeta_{3}\right)-\frac{7 x^{3}}{24 y^{3}} X^{3}+\right. \\
& +\frac{1}{12 y^{3}}(y-x)(1-x y) X\left(3 \operatorname{Li}_{2}(-x)-\pi^{2}\right)+\frac{1}{8} X^{3}+ \\
& +\frac{1}{4} Y X^{2}-\zeta_{3}-\frac{\pi^{2}}{48 y^{2}}\left(5 x^{2}+20 x+11\right)+ \\
& +\frac{1}{48 y^{3}}\left(-24 x^{2} y-77 y^{3}+21 x y^{2}+86 x^{3}\right) X^{2}+ \\
& +\frac{1}{24 y^{2}}\left(35 y^{2}+6 x^{2}-3 x y\right)\left(\operatorname{Li}_{2}(-x)+X Y\right)- \\
& -\frac{1}{144 y^{3}}\left(475 x^{3}+99 x^{2} y+279 x y^{2}-505 y^{3}\right) X+ \\
& \left.+\frac{1}{864 y^{2}}\left(32 x^{2}+5155 x+2273\right)\right] \text {, } \\
& B_{2}^{[1]}=\frac{1}{2 y^{3}}\left[\frac{x-y}{2}\left(4-5 x-5 x^{2}\right) \operatorname{Li}_{4}(-x)+\left(5 x^{3}+x^{2}-4 x+1\right) X \operatorname{Li}_{3}(-x)-\right. \\
& -\left(11 x y+x^{2}+2 y^{2}\right) \zeta_{3} X+\frac{\pi^{2}}{6}\left(17 x y^{2}+18 x^{2} y-y^{3}+2 x^{3}\right) \operatorname{Li}_{2}(-x)+ \\
& +\frac{1}{48}\left(9 y+11 y^{2}+2 y^{3}+6\right) X^{4}+\frac{\pi^{2}}{12}\left(3 x^{3}+11 x y^{2}+4 y^{3}\right) X^{2}- \\
& \left.-\frac{\pi^{4}}{1440}\left(112 x^{3}+1128 x^{2} y+916 x y^{2}+45 y^{3}\right)\right]+ \\
& +\frac{1}{y^{2}}\left[\frac{1}{2}(1+13 x) \operatorname{Li}_{4}\left(-\frac{x}{y}\right)+\frac{1}{2}\left(12 x^{2}+15 x y+7 y^{2}\right) \operatorname{Li}_{4}(-y)-\right. \\
& -(1-5 x)\left(X \operatorname{Li}_{3}(-y)-\frac{1}{2} Y\left(\operatorname{Li}_{3}(-x)-\zeta_{3}\right)\right)+ \\
& +\frac{5}{8}\left(3 x^{2}+2 y^{2}+3 x y\right) X^{2} \operatorname{Li}_{2}(-x)-\frac{1}{8}\left(31 x y+9 y^{2}+24 x^{2}\right) X^{2} Y^{2}- \\
& -2 y^{2} Y \operatorname{Li}_{3}(-y)-(2-x) X Y \operatorname{Li}_{2}(-x)-\frac{1}{48}\left(-3 y^{2}+12 x^{2}+11 x y\right) Y^{4}+ \\
& +x y \operatorname{Li}_{2}(-x) Y^{2}+\frac{1}{12}\left(6 x^{2}+11 x y+10 y^{2}\right) Y X^{3}- \\
& -\frac{1}{6}(2-y)(3 x-y) X Y^{3}-\frac{1}{6}\left(2 x^{2}-2 x-1\right) \pi^{2} Y^{2}+
\end{aligned}
$$




$$
\begin{aligned}
& \left.+\frac{1}{6}\left(15 x^{2}+13 x y+3 y^{2}\right) \pi^{2} Y X\right]+\frac{1}{2 y} \pi^{2} X^{2}+ \\
& +\frac{1}{4 y^{3}}\left[\left(4 x y+3 x^{2}+9 y^{2}\right)\left(\operatorname{Li}_{3}(-x)-X \operatorname{Li}_{2}(-x)\right)+\right. \\
& +\frac{1}{36}\left(720 x y^{2}-11 y^{3}+396 x^{2} y+108 x^{3}\right) \zeta_{3}- \\
& -\frac{1}{18}\left(120 y^{2}+60 y^{3}+43+78 y\right) X^{3}- \\
& \left.-\frac{\pi^{2}}{18}\left(91 x^{2}+140 x y+160 y^{2}-132\right) X\right]+ \\
& +\frac{1}{y^{2}}\left(-\frac{1}{12}(1-x) \pi^{2} Y+\frac{1}{24}\left(20 x^{2}-23 x-13\right) X^{2} Y\right)+ \\
& +\frac{1}{y}\left(3 \operatorname{Li}_{3}(-y)+3 \operatorname{Li}_{2}(-x) Y-\frac{1}{12}(10 x+3) X Y^{2}\right)-\frac{5}{6} Y^{3}- \\
& -\frac{1}{144 y^{3}}\left(416 x^{3}+27 x^{4}+742 x^{2}+190+902 x\right) X^{2}+ \\
& +\frac{1}{144}(191+27 x) Y^{2}-\frac{\pi^{2}}{288 y^{2}}\left(463+366 x-35 x^{2}-54 x^{3}\right)+ \\
& +\frac{1}{72 y^{2}}\left(54 x^{3}+17 x^{2} y+100 y^{3}+36 x y^{2}\right) X Y-\frac{3(y-1)}{8 y} Y- \\
& -\frac{1}{72 y^{2}}\left(291 y^{2}+305 x^{2}+383 x y\right) X-\frac{139 x}{72 y}+\frac{14135}{2592}+ \\
& +i \pi\left[\frac { 1 } { y ^ { 3 } } \left(-y\left(7 x y+3 y^{2}+6 x^{2}\right) \operatorname{Li}_{3}(-y)-\frac{x^{2}}{2}(6 y+x) \operatorname{Li}_{3}(-x)-\right.\right. \\
& -\frac{1}{2}\left(1-3 y-3 y^{2}\right) \zeta_{3}-\frac{\pi^{2}}{12}\left(2 y^{2}+8 x y+x^{2}\right) X- \\
& \left.-\frac{1}{24}\left(5 x y^{2}+4 y^{3}+4 x^{3}+3 x^{2} y\right) X^{3}\right)- \\
& -\frac{1}{y^{2}}\left(\frac{1}{4}(3+5 y)(x-y) X \operatorname{Li}_{2}(-x)+\right. \\
& +\left(3 x y+3 x^{2}+2 y^{2}\right) \operatorname{Li}_{2}(-x) Y+ \\
& \left.+\frac{1}{2}\left(6 x^{2}+5 y^{2}+8 x y\right) X Y^{2}-\frac{\pi^{2}}{12}\left(4 x^{2}+7 x+9\right) Y\right)+ \\
& +\frac{1}{4 y}\left((3 y-4) Y X^{2}+2 X Y^{2}-\frac{1}{3}(x-y) Y^{3}+2 \pi^{2} X\right)+ \\
& +\frac{1}{4 y^{3}}\left(-\mathrm{Li}_{2}(-x)\left(3 x^{2}-3 y^{2}+4 x y\right)-\right. \\
& -\frac{1}{6}\left(34+42 y+33 y^{2}+40 y^{3}\right) X^{2}+ \\
& \left.+\frac{1}{24}\left(83 x+47 x^{2}+x^{3}+49\right) \pi^{2}\right)+ \\
& +\frac{1}{12 y^{2}}\left(29 y^{2}+30 x^{2}+19 x y\right) X Y+\frac{1}{12 y}(11 x-9 y) Y^{2}+\frac{1}{96} \pi^{2}-
\end{aligned}
$$




$$
\begin{aligned}
& -\frac{1}{72 y^{3}}\left(-504 y-199 y^{2}-144 y^{3}-359\right) X+ \\
& \left.+\frac{1}{72 y^{2}}(-91 y+54 x) Y-\frac{5}{72 y^{2}}\left(48 y^{2}+40 y+61\right)\right], \\
& C_{2}^{[1]}=\frac{1}{4 y^{3}}\left[\left(2 x^{2}-8 x+2\right)\left(\operatorname{Li}_{4}(-y)-\operatorname{Li}_{4}\left(-\frac{x}{y}\right)\right)+\right. \\
& +\left(2 x^{2}-8 x-5\right) \operatorname{Li}_{4}(-x)-\frac{1}{6}\left(2 x^{2}-8 x-3\right) \pi^{2} X^{2}+ \\
& +\left(1+4 x-x^{2}\right)\left(2\left(\operatorname{Li}_{3}(-x)-\zeta_{3}\right) X-\frac{\pi^{2}}{3} \operatorname{Li}_{2}(-x)\right)- \\
& -\left(1-4 x+x^{2}\right) Y\left(\frac{\pi^{2}}{6} Y-2\left(\operatorname{Li}_{3}(-x)-\zeta_{3}\right)-\frac{1}{3} Y^{2} X+\frac{1}{12} Y^{3}\right)- \\
& -\frac{1}{2} X^{2} \operatorname{Li}_{2}(-x)-\frac{1}{24}\left(2 x^{2}-8 x+3\right) X^{4}+ \\
& \left.+\frac{1}{180}\left(11 x^{3}+12 x^{2}+117 x+24\right) \pi^{4}\right]+ \\
& +\frac{1}{8 y^{3}}\left[3\left(2 x^{2}+y^{2}-2 x y\right) X \operatorname{Li}_{2}(-x)+12\left(1-x^{2}\right) \operatorname{Li}_{3}(-y)-\right. \\
& -3\left(5 x^{2}+1+4 x\right) \operatorname{Li}_{3}(-x)+\left(29 x+41 x^{2}+15 x^{3}+9\right) \zeta_{3}+ \\
& \left.+\left(4 y^{2}+5 x^{2}+4 x y\right) \pi^{2} X\right]+ \\
& +\frac{1}{2 y^{2}}\left[3(x-1) Y \operatorname{Li}_{2}(-x)-\frac{3}{2}(1-x) X Y^{2}+\frac{1}{8}(y-2) X^{2} Y-\right. \\
& \left.-\frac{1}{6}(7 x-3) \pi^{2} Y+\frac{1}{24}(9-13 x) X^{3}\right]+ \\
& +\frac{1}{32 y^{3}}\left(-16 x^{3}-6 x^{4}+5-18 x-22 x^{2}\right) X^{2}- \\
& -\frac{1}{96 y^{2}}\left(12 x\left(3 x^{2}+5 x+8\right) X Y+\left(15-118 x-59 x^{2}-18 x^{3}\right) \pi^{2}\right)- \\
& -\frac{1}{16 y}\left(2 x+3 x^{2}+11\right) Y^{2}+\frac{1}{32 y^{2}}\left(12 x^{2}+19 x-39\right) X- \\
& -\frac{3}{8 y}(y-1) Y+\frac{187}{128}-\frac{17 x}{32 y}+ \\
& +i \pi\left[\frac{1}{8 y^{3}}\left(8 \operatorname{Li}_{3}(-x)-2 X \operatorname{Li}_{2}(-x)-X^{3}-8 \zeta_{3}\right)+\frac{1}{8 y^{2}}(y-2) X Y-\right. \\
& -\frac{1}{16 y^{3}}\left(x^{2}+4 x+9\right) X^{2}+\frac{3}{8 y^{3}}\left(x^{2}+4 x+5\right) \operatorname{Li}_{2}(-x)-\frac{1}{8 y^{3}} \pi^{2}+ \\
& \left.+\frac{1}{16 y^{3}}\left(4 x^{2}-2 x+5\right) X-\frac{1}{8 y^{2}}(5 y-6) Y-\frac{1}{32 y^{2}}(17 x+63)\right] \text {, } \\
& D_{2}^{[1]}=-\frac{1}{3} \operatorname{Li}_{2}(-x) X-\frac{7}{72 y^{3}}(x-y)(1-x y) X^{3}+\frac{1}{3} \operatorname{Li}_{3}(-x)+ \\
& +\frac{\pi^{2}}{72 y^{3}}\left(11+33 x+33 x^{2}+25 x^{3}\right) X-\frac{17}{24} \zeta_{3}-\frac{1}{6} X^{2} Y+
\end{aligned}
$$




$$
\begin{aligned}
& +\frac{1}{72 y^{3}}\left(18 x y^{2}+27 x^{2} y+37 x^{3}-43 y^{3}\right) X^{2}+ \\
& +\frac{7 \pi^{2}}{216 y^{2}}\left(20 x^{2}+25 x+11\right)+\frac{1}{36 y^{2}}\left(10+x+28 x^{2}\right) X+\frac{37 x}{72 y}+\frac{1307}{864}+ \\
& +i \pi\left[\frac{7}{24 y^{3}}(y-x)\left(x^{2}+x+1\right) X^{2}-\frac{1}{3} \operatorname{Li}_{2}(-x)-\frac{1}{3} X Y+\frac{6}{144} \pi^{2}+\right. \\
& \left.+\frac{1}{36 y^{3}}\left(71 x^{3}+138 x^{2}+147 x+43\right) X+\frac{1}{36 y^{2}}\left(28 x^{2}+x+10\right)\right], \\
& E_{2}^{[1]}=\frac{x(x-1)}{y^{3}}\left[2\left(\operatorname{Li}_{4}(-y)-\mathrm{Li}_{4}\left(-\frac{x}{y}\right)-\mathrm{Li}_{4}(-x)+\left(\operatorname{Li}_{3}(-x)-\zeta_{3}\right) Y\right)-\right. \\
& \left.-\frac{1}{3} \operatorname{Li}_{2}(-x) \pi^{2}+\frac{1}{3} Y^{3} X-\frac{1}{12} Y^{4}-\frac{1}{6} \pi^{2} Y^{2}+\frac{7}{180} \pi^{4}\right]+ \\
& +\frac{1}{y^{3}}\left[\frac{1}{3} x^{3}\left(\operatorname{Li}_{3}(-x)-\zeta_{3}\right)-\frac{1}{3} \operatorname{Li}_{2}(-x) x^{3} X-\right. \\
& \left.-\frac{1}{72}\left(6 x^{3}+3 x^{2}-7-3 x\right) X^{3}-\frac{\pi^{2}}{36}\left(4-6 x-3 x^{2}+10 x^{3}\right) X\right]+ \\
& +\frac{1}{24 y^{2}}\left[-8\left(12 x^{2}+12 y x-y^{2}\right)\left(\operatorname{Li}_{3}(-y)-\zeta_{3}+Y \operatorname{Li}_{2}(-x)\right)+\right. \\
& \left.+\left(3 x y+4 y^{2}-3 x^{2}\right) Y X^{2}-2\left(26 x y-y^{2}+24 x^{2}\right) X Y^{2}\right]- \\
& -\frac{1}{12 y}(1+4 y) \pi^{2} Y+\frac{1}{12} Y^{3}+\frac{1}{72} \zeta_{3}+\frac{1}{72 y^{3}}\left(-33 x^{2} y+x^{3}-4 x y^{2}-8 y^{3}\right) X^{2}+ \\
& +\frac{1}{18 y^{2}}\left(6 x^{2}-8 x y+5 y^{2}\right) Y X+\frac{1}{144 y^{2}}\left(84+6 y-43 y^{2}\right) \pi^{2}+ \\
& +\frac{1}{36 y x}\left(-10 x y-27 y^{2}+36 x^{2}\right) Y^{2}-\frac{1}{36 y^{2}}\left(14 x y-33 y^{2}+11 x^{2}\right) X- \\
& -\frac{1}{6 y}(5 x+9 y) Y+\frac{19 x}{72 y}-\frac{3401}{2592}+ \\
& +i \pi\left[-\frac{1}{3 y^{3}} \operatorname{Li}_{2}(-x)\left(12 x y^{2}-y^{3}+x^{3}+12 x^{2} y\right)+\right. \\
& +\frac{2 x(x-1)}{y^{3}}\left(\operatorname{Li}_{3}(-x)-\zeta_{3}\right)+\frac{1}{8 y^{3}}(1+2 x y) X^{2}+ \\
& +\frac{1}{18 y^{3}} \pi^{2} x^{3}+\frac{1}{12 y^{2}}(3 x-2 y) X Y- \\
& -\frac{1}{6 y}(x-y) Y^{2}-\frac{1}{18 y^{2} x}\left(6 x^{2}+22 x y-27 y^{2}\right) Y- \\
& \left.-\frac{1}{36 y^{3}}\left(x^{2}+14 y^{2}-10 x y+12 y\right) X+\frac{1}{36 y^{2}}\left(12 x^{2}+2 x-21\right)\right] \text {, } \\
& F_{2}^{[1]}=\frac{1}{24} X^{2}-\frac{5}{54} X-\frac{\pi^{2}}{27}+i \pi\left(\frac{1}{12} X-\frac{5}{54}\right), \\
& G_{2}^{[1]}=-\frac{x}{72 y} X^{2}+\frac{x-y}{72 y} X Y+\frac{1}{72} Y^{2}+\frac{i \pi}{72 y}(X-Y) .
\end{aligned}
$$


For $h=2$ in eq. (2.23) and color factor $\operatorname{Tr}^{[2]}$ in eq. (2.30):

$$
\begin{aligned}
& A_{2}^{[2]}=\frac{1}{y^{3}}\left[x(4 x-1)\left(\operatorname{Li}_{3}(-x) Y-\operatorname{Li}_{4}(-x)-\operatorname{Li}_{4}\left(-\frac{x}{y}\right)\right)-\right. \\
& -\frac{1}{24}\left(4 x^{3}-5 x^{2}+14 x+3\right) X Y^{3}+\frac{1}{96}\left(10 x^{3}+11 x^{2}+28 x+7\right) Y^{4}+ \\
& +\frac{\pi^{2}}{6}\left(x^{2}-2 x+2\right) x \operatorname{Li}_{2}(-x)+\frac{1}{4}\left(2 x^{3}+23 x^{2}+4 x+3\right) \operatorname{Li}_{4}(-y)- \\
& -\frac{\pi^{2}}{48}\left(22 x^{3}+75 x^{2}+48 x+15\right) Y^{2}-\frac{1}{2}\left(x^{3}+9 x^{2}-3 x-1\right) \zeta_{3} Y- \\
& \left.-\frac{\pi^{4}}{2880}\left(9 x^{3}-182 x^{2}+113 x+24\right)\right]-\frac{x-y}{8 y} Y^{2} \operatorname{Li}_{2}(-x)+\frac{1}{2} Y \operatorname{Li}_{3}(-y)+ \\
& +\frac{1}{y^{2}}\left[\frac{1}{6}\left(11 x^{2}+50 x+9\right)\left(\operatorname{Li}_{3}(-y)+\operatorname{Li}_{2}(-x) Y+\frac{1}{2} X Y^{2}\right)-\right. \\
& \left.-\frac{1}{48}\left(205 x^{2}+517 x+72\right) \zeta_{3}\right]- \\
& -\frac{13(x-y)}{18 y} Y^{3}+\frac{\pi^{2}}{144 y}(445 x+208) Y+\frac{1}{288 x y}\left(1160 x^{2}-713 x-360\right) Y^{2}+ \\
& +\frac{5 x}{6 y^{2}}(3 x+2) \pi^{2}+\frac{2 x+135}{54 y} Y+\frac{x(30377 x+30593)}{3456 y^{2}}+ \\
& +i \pi\left[\frac{1}{y^{3}}\left((4 x-1) x \operatorname{Li}_{3}(-x)-\frac{1}{2}\left(x^{3}+9 x^{2}-3 x-1\right) \zeta_{3}\right)-\right. \\
& -\frac{x-y}{4 y} Y\left(\operatorname{Li}_{2}(-x)+\frac{\pi^{2}}{6}\right)+\frac{10 x+7}{24 y} Y^{3}+\frac{1}{2} \operatorname{Li}_{3}(-y)+ \\
& +\frac{1}{4} X Y^{2}+\frac{1}{6 y^{2}}\left(11 x^{2}+50 x+9\right) \operatorname{Li}_{2}(-x)-\frac{13(x-y)}{6 y} Y^{2}+ \\
& \left.+\frac{29 x}{144 y} \pi^{2}-\frac{1}{144 x y}\left(-1160 x^{2}+713 x+360\right) Y+\frac{133}{54 y}-\frac{1}{27}\right], \\
& B_{2}^{[2]}=\frac{1}{2 y^{3}}\left[\left(4 x^{3}+12 x^{2}-3\right) \operatorname{Li}_{4}(-x)+\left(5 x^{3}+4 x^{2}-x+7\right) \operatorname{Li}_{4}(-y)+\right. \\
& +\left(14 x^{2}+22 x+1\right) \operatorname{Li}_{4}\left(-\frac{x}{y}\right)-\left(5 x^{2}+10 x-2\right) Y \operatorname{Li}_{3}(-x)- \\
& -2\left(2 x^{2}+x-4\right) x X \operatorname{Li}_{3}(-x)+\frac{x}{12}\left(x^{2}-x+1\right) X^{4}+ \\
& +\frac{\pi^{2}}{6}\left(2 x^{3}-6 x^{2}-12 x+1\right) \operatorname{Li}_{2}(-x)+ \\
& +\frac{1}{24}\left(x^{3}+20 x^{2}+31 x+5\right) Y^{4}-\frac{1}{6}\left(10 x^{3}+39 x^{2}+42 x+6\right) X Y^{3}- \\
& -\frac{\pi^{2}}{12}\left(5 x^{3}+10 x^{2}+11 x+13\right) Y^{2}-\frac{\pi^{2}}{4}\left(4 x^{3}+4 x^{2}-3 x+1\right) X^{2}- \\
& -\left(5 x^{2}+10 x-1\right) \zeta_{3} X+\left(5 x^{2}+10 x-2\right) \zeta_{3} Y- \\
& \left.-\frac{\pi^{4}}{1440}\left(225 x^{3}-130 x^{2}-719 x+112\right)\right]+
\end{aligned}
$$




$$
\begin{aligned}
& +\frac{1}{2 y^{2}}\left[-(11 x-1) X \operatorname{Li}_{3}(-y)-\left(2 x^{2}+x+2\right) X^{2} \operatorname{Li}_{2}(-x)-\right. \\
& -2(x-2) X Y \operatorname{Li}_{2}(-x)-\frac{1}{6}\left(4 x^{2}+7 x+9\right) X^{3} Y+ \\
& \left.+\frac{1}{2}\left(x^{2}-7 x+4\right) X^{2} Y^{2}-\frac{\pi^{2}}{3}\left(6 x^{2}-5 x+4\right) X Y\right]+ \\
& +\frac{1}{2 y}\left(-(5 x+4) Y \operatorname{Li}_{3}(-y)-\frac{5}{2} x Y^{2} \operatorname{Li}_{2}(-x)+\pi^{2}\left(Y^{2}-X^{2}\right)\right)+ \\
& +\frac{1}{4 y^{3}}\left[\left(21 x^{2}+26 x+12\right)\left(X \operatorname{Li}_{2}(-x)-\operatorname{Li}_{3}(-x)\right)+\right. \\
& +\frac{5 \pi^{2}}{6}\left(7 x^{2}+10 x+4\right) X-\frac{x}{3}\left(10 x^{2}+5 x+1\right) X^{3}+ \\
& +\frac{1}{6}\left(20 x^{3}+15 x^{2}-12 x-4\right) X^{2} Y- \\
& \left.-\frac{1}{36}\left(443 x^{3}+706 x^{2}+299 x-216\right) \zeta_{3}\right]+ \\
& +\frac{1}{2 y^{2}}\left[(10 x+3)\left(\operatorname{Li}_{3}(-y)+Y \operatorname{Li}_{2}(-x)\right)-\right. \\
& \left.-\frac{1}{6}\left(10 x^{2}+x+12\right) X Y^{2}-\frac{2 \pi^{2}}{9}(19 x+10) Y\right]- \\
& -\frac{15 x+28}{18 y} Y^{3}-\frac{x}{144 y^{3}}\left(27 x^{3}-92 x^{2}-184 x-164\right) X^{2}- \\
& -\frac{1}{288 y^{2}}\left[4\left(27 x^{3}-119 x^{2}-138 x-100\right) X Y-\pi^{2} x\left(54 x^{2}+217 x+343\right)\right]- \\
& -\frac{1}{144 x y}\left(27 x^{3}-290 x^{2}-199 x+72\right) Y^{2}-\frac{3 x(y-1)}{8 y^{2}} X- \\
& -\frac{71 x-24}{24 y} Y-\frac{x}{2592 y^{2}}(19139 x+18815)+ \\
& +i \pi\left[-\frac{1}{2 y^{3}}\left(\left(4 x^{3}+7 x^{2}+2 x-2\right) \operatorname{Li}_{3}(-x)+\zeta_{3}\right)+\right. \\
& +\frac{1}{2 y^{2}}\left[\left(5 x^{2}-2 x+5\right) \operatorname{Li}_{3}(-y)+\left(5 x^{2}+3 x+4\right) Y \operatorname{Li}_{2}(-x)-\right. \\
& -\frac{1}{6}(2 x-1)(x-1) X^{3}+\frac{1}{2}\left(7 x^{2}+7 x+12\right) X Y^{2}+ \\
& \left.+\frac{\pi^{2}}{6}(8 x+5) X-\frac{\pi^{2}}{6}\left(5 x^{2}+7 x+8\right) Y\right]+ \\
& +\frac{1}{y}\left(2 x X \operatorname{Li}_{2}(-x)+\frac{1}{12}(x+3) Y^{3}-\frac{1}{2}(y-2) X^{2} Y\right)+ \\
& +\frac{1}{4 y^{3}}\left[\left(x^{2}+6\right) \operatorname{Li}_{2}(-x)-\frac{1}{3}\left(20 x^{3}+39 x^{2}+48 x+20\right) X^{2}+\right. \\
& +\frac{1}{3}\left(40 x^{3}+97 x^{2}+92 x+38\right) X Y- \\
& \left.-\frac{\pi^{2}}{24}\left(x^{3}+86 x^{2}+153 x+96\right)\right]-
\end{aligned}
$$




$$
\begin{aligned}
& -\frac{5(4 x+7)}{12 y} Y^{2}+\frac{x}{96 y} \pi^{2}-\frac{1}{72 y^{3}}\left(73 x^{2}+74 x+100\right) X- \\
& \left.-\frac{1}{8 x y^{2}}\left(16 x^{3}+39 x^{2}+3 x-8\right) Y+\frac{1}{24 y^{2}}\left(80 x^{2}+65 x-24\right)\right] \text {, } \\
& C_{2}^{[2]}=\frac{1}{2 y^{3}}\left[\left(2 x^{2}+4 x+1\right)\left(\operatorname{Li}_{4}(-x)+\operatorname{Li}_{4}\left(-\frac{x}{y}\right)+\frac{\pi^{2}}{6} \operatorname{Li}_{2}(-x)\right)-\right. \\
& -\frac{1}{2}\left(7 x^{2}+14 x+5\right) \operatorname{Li}_{4}(-y)+x(y-1) Y \operatorname{Li}_{3}(-x)+ \\
& +\frac{\pi^{2}}{24}\left(9 x^{2}+18 x+7\right) Y^{2}-\zeta_{3} Y-\frac{1}{12}\left(x^{2}+2 x-1\right)\left(X Y^{3}-\frac{1}{4} Y^{4}\right)- \\
& \left.-\frac{\pi^{4}}{360}\left(11 x^{3}+19 x^{2}+5 x-10\right)\right]+ \\
& +\frac{1}{2 y}\left(X\left(\operatorname{Li}_{3}(-y)-\zeta_{3}\right)+Y \operatorname{Li}_{3}(-y)+\frac{1}{4} Y^{2} \operatorname{Li}_{2}(-x)+\frac{1}{4} X^{2} Y^{2}\right)+ \\
& +\frac{x(3 y+1)}{4 y^{3}}\left(\operatorname{Li}_{3}(-x)-X \operatorname{Li}_{2}(-x)-\frac{1}{2} X^{2} Y+\frac{\pi^{2}}{6} X\right)- \\
& -\frac{5 x+4}{8 y^{3}}\left(3 x^{2}+4 x+3\right) \zeta_{3}+\frac{x}{96 y^{2}}\left(18 x^{2}+25 x-5\right) \pi^{2}+ \\
& +\frac{2 y-1}{4 y^{2}}\left(2 \operatorname{Li}_{3}(-y)+2 \operatorname{Li}_{2}(-x) Y+X Y^{2}\right)- \\
& -\frac{x}{16 y^{3}}\left(3 x^{3}+12 x^{2}+12 x+4\right) X^{2}-\frac{3 x(y-1)}{8 y^{2}} X- \\
& -\frac{1}{32 x y}\left(6 x^{3}+12 x^{2}-15 x-8\right) Y^{2}-\frac{3 x(y-1)}{8 y} X Y+ \\
& +\frac{3 x-4}{8 y} Y-\frac{x(255 x+247)}{128 y^{2}}+ \\
& +i \pi\left[\frac{1}{2 y^{3}}\left((y-1) x \operatorname{Li}_{3}(-x)-\left(3 x^{3}+7 x^{2}+5 x+2\right) \zeta_{3}\right)+\right. \\
& +\frac{1}{4 y}\left(4 \operatorname{Li}_{3}(-y)+\operatorname{Li}_{2}(-x) Y-\frac{\pi^{2}}{6} Y+X Y^{2}-\frac{1}{2} Y^{3}+6 x \zeta_{3}\right)+ \\
& +\frac{1}{4 y^{3}}\left(-(3 y+1) x X Y-\frac{\pi^{2}}{6} x(3 x+2)+\left(7 x^{2}+12 x+6\right) \operatorname{Li}_{2}(-x)\right)- \\
& \left.-\frac{x(3 y+1)}{8 y^{3}} X+\frac{15 x+8}{16 x y} Y+\frac{1}{8 y^{2}}(7 x+4)\right] \text {, } \\
& D_{2}^{[2]}=\frac{x(x-1)}{y^{3}}\left[\operatorname{Li}_{4}(-x)-\operatorname{Li}_{4}(-y)+\operatorname{Li}_{4}\left(-\frac{x}{y}\right)+\frac{\pi^{2}}{6} \mathrm{Li}_{2}(-x)+\right. \\
& \left.+\frac{1}{24} Y^{4}-\frac{1}{6} X Y^{3}+\frac{\pi^{2}}{12} Y^{2}-Y\left(\operatorname{Li}_{3}(-x)-\zeta_{3}\right)-\frac{7 \pi^{4}}{360}\right]+ \\
& +\frac{x}{6 y^{2}}\left[-\frac{1}{4}(8 x+56) \operatorname{Li}_{3}(-y)-2(x+7) \operatorname{Li}_{2}(-x) Y+\right. \\
& \left.+\frac{1}{4}(17 x+65) \zeta_{3}-(x+7) X Y^{2}\right]+\frac{7(x-y)}{72 y} Y^{3}-
\end{aligned}
$$




$$
\begin{aligned}
& -\frac{\pi^{2}}{72 y}(29 x+14) Y-\frac{1}{72 x y}\left(71 x^{2}-71 x-36\right) Y^{2}- \\
& -\pi^{2} \frac{x(35 x+17)}{54 y^{2}}+\frac{7 x-9}{9 y} Y+\frac{863 x}{864 y}+ \\
& +i \pi\left[\frac{(1-x) x}{y^{3}}\left(\operatorname{Li}_{3}(-x)-\zeta_{3}\right)-\frac{x(x+7)}{3 y^{2}} \operatorname{Li}_{2}(-x)+\frac{7(x-y)}{24 y} Y^{2}-\right. \\
& \left.-\pi^{2} \frac{x}{72 y}-\frac{1}{36 x y}\left(71 x^{2}-71 x-36\right) Y-\frac{16}{9 y}-\frac{7}{9}\right] \text {, } \\
& E_{2}^{[2]}=\frac{x(x-1)}{y^{3}}\left[-2 \mathrm{Li}_{4}(-x)+2 \operatorname{Li}_{4}(-y)-2 \operatorname{Li}_{4}\left(-\frac{x}{y}\right)+2 Y\left(\operatorname{Li}_{3}(-x)-\zeta_{3}\right)-\right. \\
& \left.-\frac{\pi^{2}}{3} \mathrm{Li}_{2}(-x)-\frac{1}{12} Y^{4}+\frac{1}{3} X Y^{3}-\frac{\pi^{2}}{6} Y^{2}+\frac{7 \pi^{4}}{180}\right]+ \\
& +\frac{1}{3 y^{3}}\left[x^{3}\left(\operatorname{Li}_{3}(-x)-X \operatorname{Li}_{2}(-x)\right)-\frac{\pi^{2}}{12}\left(10 x^{3}+3 x^{2}+6 x+3\right) X+\right. \\
& \left.+\frac{1}{24}\left(x^{3}+362 x^{2}+361 x+24\right) \zeta_{3}\right]+ \\
& +\frac{1}{3 y^{2}}\left[\left(x^{2}+14 x+1\right)\left(\operatorname{Li}_{3}(-y)+\operatorname{Li}_{2}(-x) Y\right)-\frac{1}{8} x(2 x-1) X^{3}+\right. \\
& \left.+\frac{1}{8}\left(2 x^{2}-5 x-4\right) X^{2} Y+\frac{1}{4}(x+7)(5 x+1) X Y^{2}\right]+ \\
& +\frac{1}{9 y}\left(\frac{1}{8}(6 x+13) Y^{3}+(3 x+2) \pi^{2} Y\right)-\frac{x}{36 y^{3}}\left(x^{2}+20 x+28\right) X^{2}- \\
& -\frac{1}{18 y^{2}}\left(x^{2}+12 x+5\right) X Y-\pi^{2} \frac{x}{144 y^{2}}(29 x+85)+\frac{x(4 x+9)}{6 y^{2}} X- \\
& -\frac{1}{72 x y}\left(2 x^{2}+147 x+54\right) Y^{2}+\frac{2 x+3}{2 y} Y-\frac{4085 x}{2592 y}+ \\
& +i \pi\left[2 \frac{x(x-1)}{y^{3}}\left(\operatorname{Li}_{3}(-x)-\zeta_{3}\right)+\frac{1}{12 y^{2}}\left(8 x^{2}+11 x+6\right) X Y-\right. \\
& -\frac{1}{3 y^{3}}\left(\left(2 x^{3}+15 x^{2}+15 x+1\right) \operatorname{Li}_{2}(-x)-\right. \\
& \left.-\frac{1}{4}\left(4 x^{3}+3 x^{2}+3 x+2\right) X^{2}-\frac{\pi^{2}}{6} x^{3}\right)+ \\
& +\frac{1}{8 y} Y^{2}+\frac{1}{36 x y^{2}}\left(125 x^{2}+191 x+54\right) Y- \\
& \left.-\frac{1}{18 y^{3}}\left(7 x^{2}+11 x-5\right) X-\frac{1}{6 y^{2}}\left(2 x^{2}+6 x+9\right)\right] \text {, } \\
& F_{2}^{[2]}=\frac{x}{y}\left[\frac{1}{24} Y^{2}-\frac{\pi^{2}}{27}-\frac{5}{54} Y+i \pi\left(\frac{1}{12} Y-\frac{5}{54}\right)\right], \\
& G_{2}^{[2]}=\frac{1}{72}\left[\frac{x}{y} X^{2}-\frac{2 x+1}{y} X Y-Y^{2}+i \frac{\pi}{y}(Y-X)\right] .
\end{aligned}
$$


For $h=2$ in eq. (2.23) and color factor $\operatorname{Tr}^{[3]}$ in eq. (2.30):

$$
\begin{aligned}
& H_{2}^{[3]}=\frac{1}{2 y^{3}}\left[\left(5 x^{3}+56 x^{2}+31 x+13\right) \operatorname{Li}_{4}(-y)-\frac{1}{2}\left(10 x^{3}-16-47 x+23 x^{2}\right) \operatorname{Li}_{4}(-x)+\right. \\
& +\left(16 x^{3}+7 x^{2}+32 x+8\right) \operatorname{Li}_{4}\left(-\frac{x}{y}\right)- \\
& -\left(12 x^{3}+2 x^{2}+28 x+7\right) Y \operatorname{Li}_{3}(-x)+ \\
& +\left(12 x^{3}+21 x^{2}+18 x+5\right)\left(X \operatorname{Li}_{3}(-y)+\frac{1}{2} X^{2} Y^{2}\right)+ \\
& +\left(13 x^{3}+10 x^{2}-2+2 x\right) X \operatorname{Li}_{3}(-x)- \\
& -\frac{1}{4}\left(10 x^{3}+3 x^{2}-9 x-8\right) X^{2} \operatorname{Li}_{2}(-x)+ \\
& +2(x-y)\left(2 x^{2}+3 x+2\right) X Y \operatorname{Li}_{2}(-x)- \\
& -\frac{\pi^{2}}{6}\left(44 x^{3}+109 x^{2}+68 x+22\right) \operatorname{Li}_{2}(-x)- \\
& -\frac{1}{6}\left(32 x^{3}+63 x^{2}+96 x+32\right) X Y^{3}+\frac{1}{6}\left(x^{3}+10 x^{2}+11 x+4\right) X^{3} Y+ \\
& +\frac{1}{24}\left(19 x^{3}+16 x^{2}+41 x+11\right) Y^{4}+\frac{\pi^{2}}{4}\left(10 x^{3}+11 x^{2}+13 x+8\right) X^{2}- \\
& -\frac{\pi^{2}}{3}\left(17 x^{3}+30 x^{2}+24 x+6\right) X Y-\frac{\pi^{2}}{12}\left(9 x^{3}+40 x^{2}-13 x-11\right) Y^{2}- \\
& -\left(14 x+13 x^{2}+11 x^{3}+5\right) \zeta_{3} X+\left(11 x^{3}-x^{2}+25 x+6\right) \zeta_{3} Y+ \\
& \left.+\frac{\pi^{4}}{360}\left(276 x^{3}+147 x^{2}-378 x-202\right)\right]+\frac{x(2 x-1)}{32 y^{2}} X^{4}- \\
& -\frac{1}{2 y}\left((13 x+15) Y \operatorname{Li}_{3}(-y)+\frac{1}{2}(5 x+9) Y^{2} \operatorname{Li}_{2}(-x)+(2 x+1) \pi^{2}\left(X^{2}-Y^{2}\right)\right)+ \\
& +\frac{1}{12 y^{3}}\left[\left(62 x^{3}+67 x^{2}+62 x+26\right)\left(\operatorname{Li}_{3}(-x)-X \operatorname{Li}_{2}(-x)\right)+\right. \\
& +\left(242 x^{2}+238 x+27\right) \zeta_{3}+\frac{x}{3}\left(22 x^{2}+14 x-11\right) X^{3}- \\
& -\left(21 x^{3}+48 x^{2}+39 x+16\right) X^{2} Y- \\
& \left.-\frac{\pi^{2}}{12}\left(321 x^{3}-182 x^{2}-139 x-18\right) X\right]+ \\
& +\frac{1}{12 y^{2}}\left[\left(62 x^{2}+263 x+27\right)\left(\operatorname{Li}_{3}(-y)+Y \operatorname{Li}_{2}(-x)\right)+\right. \\
& \left.+\left(41 x^{2}+132 x+4\right) X Y^{2}-\frac{\pi^{2}}{12}\left(445 x^{2}+418 x-75\right) Y\right]+ \\
& +\frac{11}{18} Y^{3}-\frac{x}{72 y^{3}}\left(172 x^{2}+404 x+97\right) X^{2}+\frac{x-2}{12 y^{2}}(6 x-11) X Y+ \\
& +\frac{1}{72 x y}\left(208 x^{2}-161 x-108\right) Y^{2}-\frac{\pi^{2}}{72 y^{2}}\left(18 x^{2}-108 x-49\right)+ \\
& +\frac{x}{54 y^{2}}(38 x+83) X+\frac{1}{108 y}(76 x+211) Y+
\end{aligned}
$$




$$
\begin{aligned}
& +i \pi\left[\frac { 1 } { 2 y ^ { 3 } } \left(\left(x^{3}+8 x^{2}-26 x-9\right) \operatorname{Li}_{3}(-x)-\left(x^{3}+20 x^{2}+25 x+10\right) \operatorname{Li}_{3}(-y)+\right.\right. \\
& +\left(3 x^{3}-3 x^{2}-9 x-5\right) Y \operatorname{Li}_{2}(-x)+ \\
& +\frac{1}{2}\left(6 x^{3}+29 x^{2}+37 x+16\right) X \operatorname{Li}_{2}(-x)+ \\
& +\frac{1}{2}\left(5 x^{3}-6 x^{2}-15 x-8\right) X Y^{2}- \\
& -\frac{1}{12}\left(10 x^{3}-3 x^{2}-3 x+4\right) X^{3}-\left(14 x^{2}-11 x-1\right) \zeta_{3}- \\
& \left.-\frac{\pi^{2}}{6}\left(3 x^{3}-15 x^{2}-33 x-17\right) Y+\frac{\pi^{2}}{3}\left(5 x^{2}+10 x+6\right) X\right)+ \\
& +\frac{1}{12 y}\left((5 x+3) Y^{3}-15 y X^{2} Y-6(2 x+1)\left(X Y^{2}+\pi^{2}(X-Y)\right)\right)+ \\
& +\frac{1}{12 y^{3}}\left(-\left(124 x^{3}+392 x^{2}+352 x+53\right) \operatorname{Li}_{2}(-x)+\right. \\
& +\frac{1}{2}\left(64 x^{3}-x^{2}-38 x-6\right) X^{2}- \\
& -\left(62 x^{3}+117 x^{2}+60 x+13\right) X Y+ \\
& \left.+\frac{\pi^{2}}{12}\left(124 x^{3}+29 x^{2}-110 x-77\right)\right)- \\
& -\frac{64 x+25}{24 y} Y^{2}-\frac{1}{36 y^{3}}\left(190 x^{3}+353 x^{2}+94 x+66\right) X- \\
& \left.-\frac{1}{36 x y^{2}}\left(190 x^{3}+116 x^{2}-335 x-108\right) Y-\frac{121 x+211}{108 y^{2}}\right], \\
& I_{2}^{[3]}=\frac{1}{2 y^{3}}\left[\left(x^{3}+4 x^{2}+5 x+5\right) \operatorname{Li}_{4}(-y)-\left(3 x^{2}+9 x-1\right) \zeta_{3} X-\right. \\
& -\frac{1}{2}\left(2 x^{3}+3 x^{2}+9 x-8\right) \operatorname{Li}_{4}(-x)-3(y-1) x \operatorname{Li}_{4}\left(-\frac{x}{y}\right)- \\
& -\left(2 x^{2}+4 x+1\right) Y\left(\operatorname{Li}_{3}(-x)-\zeta_{3}\right)-(x+3) y^{2} Y \operatorname{Li}_{3}(-y)+ \\
& +\left(3 x^{2}+6 x-1\right) X \operatorname{Li}_{3}(-y)+\frac{1}{2} y^{3} Y^{2} \operatorname{Li}_{2}(-x)+ \\
& +\left(x^{3}+4 x^{2}+8 x-2\right) X \operatorname{Li}_{3}(-x)-2 x(y-1) \operatorname{Li}_{2}(-x) X Y+ \\
& +\frac{\pi^{2}}{6}\left(4 x^{3}-x^{2}-14 x+2\right) \operatorname{Li}_{2}(-x)-\frac{1}{24}\left(x^{3}-3 x+1\right) Y^{4}- \\
& -\frac{x}{4}\left(2 x^{2}+7 x+11\right) X^{2} \operatorname{Li}_{2}(-x)+\frac{1}{4}\left(5 x^{2}+10 x-3\right) X^{2} Y^{2}- \\
& -\frac{1}{6}\left(x^{3}+3 x^{2}+3 x-1\right) X^{3} Y-\frac{\pi^{2}}{3}(y-1)\left(x^{2}-3 x+1\right) X Y- \\
& -\frac{\pi^{2}}{12}\left(2 x^{3}+x^{2}+2 x+15\right) X^{2}+\frac{\pi^{2}}{4}\left(x^{3}-x^{2}-5 x-4\right) Y^{2}- \\
& -\frac{1}{6}\left(2 x^{3}+8 x^{2}+10 x+1\right) X Y^{3}-\frac{x y}{48}(2 x-1) X^{4}- \\
& \left.-\frac{\pi^{4}}{360}\left(20 x^{3}-85 x^{2}-248 x+20\right)\right]+
\end{aligned}
$$




$$
\begin{aligned}
& +\frac{1}{4 y^{3}}\left[-\left(x^{2}+4 x-6\right)\left(\operatorname{Li}_{3}(-x)-X \operatorname{Li}_{2}(-x)\right)+\frac{1}{3} x^{2} X^{3}+\right. \\
& \left.+\frac{1}{3}(9 x+5) X^{2} Y+9(x-y) \zeta_{3}-\frac{\pi^{2}}{6}(14 x+47) X\right]+ \\
& +\frac{1}{y^{2}}\left[\frac{1}{4}(7 x+9)\left(\operatorname{Li}_{3}(-y)+\operatorname{Li}_{2}(-x) Y\right)+\frac{1}{12}(10 x+13) X Y^{2}+\frac{\pi^{2}}{3} Y\right]+ \\
& +\frac{x}{8 y^{3}}\left(2 x^{3}+6 x+9\right) X^{2}+\frac{1}{12 y^{2}}\left(6 x^{3}+18 x^{2}+25 x+6\right) X Y+ \\
& +\frac{1}{8 x y}\left(2 x^{3}+12 x^{2}+9 x-2\right) Y^{2}-\frac{\pi^{2}}{24 y^{2}}\left(6 x^{3}+18 x^{2}+14 x-9\right)- \\
& -\frac{x}{6 y^{2}}(23 x+26) X-\frac{1}{12 y}(46 x+47) Y+ \\
& +i \pi\left[\frac { 1 } { 2 y ^ { 3 } } \left(-\left(x^{3}+2 x^{2}+x+4\right) \operatorname{Li}_{3}(-y)-\frac{\pi^{2}}{6}(x+3)(x-y) X+\right.\right. \\
& +\left(x^{3}+2 x^{2}+4 x-3\right) \operatorname{Li}_{3}(-x)-\left(x^{2}+5 x-2\right) \zeta_{3}+ \\
& +\frac{1}{12}\left(2 x^{3}+x^{2}-x+6\right) X^{3}+\frac{1}{2}(y-1)(x-1)^{2} X Y^{2}- \\
& -\frac{x}{2}\left(2 x^{2}+3 x+3\right) X \operatorname{Li}_{2}(-x)- \\
& -\left(x^{3}+x^{2}-x+1\right) Y \operatorname{Li}_{2}(-x)+ \\
& \left.+\frac{\pi^{2}}{6}\left(x^{3}-2 x^{2}-7 x-2\right) Y\right)-\frac{x-2}{12 y} Y^{3}+\frac{1}{4} X^{2} Y+ \\
& +\frac{1}{4 y^{3}}\left(-3\left(2 x^{2}+4 x+5\right) \operatorname{Li}_{2}(-x)+\frac{1}{6}\left(3 x^{2}+6 x+28\right) X^{2}+\right. \\
& \left.+\frac{1}{3}\left(x^{2}+20 x+11\right) X Y-\frac{\pi^{2}}{2}(2 x-1)\right)+ \\
& +\frac{1}{24 y} Y^{2}-\frac{1}{12 y^{3}}\left(24 x^{3}+25 x^{2}+4 x+6\right) X- \\
& \left.-\frac{1}{12 x y^{2}}\left(24 x^{3}+38 x^{2}+15 x-6\right) Y+\frac{1}{12 y^{2}}(41 x+47)\right] \text {, } \\
& J_{2}^{[3]}=\frac{(x-1) x}{y^{3}}\left[\operatorname{Li}_{4}(-x)+\operatorname{Li}_{4}\left(-\frac{x}{y}\right)-\operatorname{Li}_{4}(-y)-Y \mathrm{Li}_{3}(-x)+\right. \\
& \left.+\frac{\pi^{2}}{6} \operatorname{Li}_{2}(-x)-\frac{1}{6} X Y^{3}+\frac{1}{24} Y^{4}+\frac{\pi^{2}}{12} Y^{2}+\zeta_{3} Y-\frac{7 \pi^{4}}{360}\right]+ \\
& +\frac{1}{24 y^{3}}\left[\left(6 x^{3}+15 x^{2}+15 x+5\right) X^{2} Y+\frac{\pi^{2}}{3}\left(x^{3}+8 x^{2}+19 x+18\right) X\right]+ \\
& +\frac{1}{3 y^{2}}\left[-\left(x^{2}+6 x-1\right)\left(\operatorname{Li}_{3}(-y)+Y \operatorname{Li}_{2}(-x)\right)+\frac{1}{6}(2 x-1) x X^{3}-\right. \\
& \left.-\frac{1}{8}(x+9)(2 x-1) X Y^{2}+(5 x-1) \zeta_{3}\right]+ \\
& +\frac{1}{3 y}\left((y-1)\left(\operatorname{Li}_{3}(-x)-X \operatorname{Li}_{2}(-x)\right)-\frac{\pi^{2}}{24}(5 x-21) Y\right)-
\end{aligned}
$$




$$
\begin{aligned}
& -\frac{1}{9} Y^{3}+\frac{x}{24 y^{3}}\left(6 x^{3}+41 x^{2}+48 x+19\right) X^{2}+ \\
& +\frac{1}{72 y^{2}}\left(36 x^{3}+108 x^{2}+37 x-29\right) X Y+\frac{(5 x-4) x}{18 y^{2}} X+ \\
& +\frac{1}{24 x y}\left(6 x^{3}-5 x^{2}+13 x+12\right) Y^{2}+\frac{(20 x-19)}{72 y} Y- \\
& -\frac{\pi^{2}}{36 y^{2}}\left(9 x^{3}+27 x^{2}+14 x+11\right)+ \\
& +i \pi\left[-\frac{(x-1) x}{y^{3}}\left(\operatorname{Li}_{3}(-x)-\zeta_{3}\right)-\frac{1}{3 y^{2}}\left(2 x^{2}+9 x+1\right) \operatorname{Li}_{2}(-x)+\right. \\
& +\frac{1}{24 y^{3}}\left(2\left(4 x^{2}+6 x+3\right) x X Y-\left(6 x^{3}+5 x^{2}+x+3\right) X^{2}\right)+ \\
& +\frac{1}{72 y^{3}}\left(102 x^{3}+143 x^{2}+106 x+29\right) X+ \\
& +\frac{1}{72 x y^{2}}\left(102 x^{3}-11 x^{2}-179 x-72\right) Y+ \\
& \left.+\frac{x-y}{8 y} Y^{2}-\frac{\pi^{2}}{72 y}(4 x-7)-\frac{17 x-19}{72 y^{2}}\right], \\
& K_{2}^{[3]}=\frac{1}{y^{3}}\left[2\left(\operatorname{Li}_{4}(-x)+\operatorname{Li}_{4}\left(-\frac{x}{y}\right)-\mathrm{Li}_{4}(-y)\right)(x-1) x+\right. \\
& +2\left(-\mathrm{Li}_{3}(-x)+\zeta_{3}\right)(x-1) x Y+\frac{\pi^{2}}{3}(x-1) x \operatorname{Li}_{2}(-x)- \\
& \left.-\frac{x}{3}(x-1) X Y^{3}+\frac{x}{12}(x-1) Y^{4}+\frac{\pi^{2}}{6}(x-1) x Y^{2}-\frac{7 \pi^{4}}{180}(x-1) x\right]+ \\
& +\frac{1}{3 y^{3}}\left[-x^{3} \operatorname{Li}_{3}(-x)+x^{3} X \operatorname{Li}_{2}(-x)-\frac{1}{8} X^{2} Y-\right. \\
& \left.-\left(15 x^{2}+15 x+1\right) \zeta_{3}+\frac{\pi^{2}}{12}\left(10 x^{3}+3\right) X\right]- \\
& -\frac{1}{3 y^{2}}\left(x^{2}+14 x+1\right)\left(\operatorname{Li}_{3}(-y)+Y \operatorname{Li}_{2}(-x)\right)- \\
& -\frac{1}{24 y^{2}}\left(8 x^{2}+63 x+7\right) X Y^{2}+\frac{\pi^{2}}{12 y}(4 y+1) Y- \\
& -\frac{1}{8 y^{2}}\left(\left(4 x^{2}+x-1\right) X Y-\frac{\pi^{2}}{9}\left(18 x^{2}+43 x-9\right)\right)- \\
& -\frac{1}{4 y^{3}}\left(x^{2}-2\right) x X^{2}-\frac{1}{4 x y}\left(x^{2}-6 x-3\right) Y^{2}-\frac{8 x+29}{24 y} Y-\frac{x}{6 y^{2}}(2 x+7) X+ \\
& +i \pi\left[2 \frac{(x-1) x}{y^{3}}\left(-\mathrm{Li}_{3}(-x)+\zeta_{3}\right)+\right. \\
& +\frac{1}{3 y^{3}}\left(\left(2 x^{3}+15 x^{2}+15 x+1\right) \operatorname{Li}_{2}(-x)-\frac{1}{8}\left(4 x^{3}+1\right) X^{2}+\right. \\
& \left.+\frac{1}{4}\left(4 x^{3}+11 x^{2}+10 x+2\right) X Y-\frac{\pi^{2}}{6} x^{3}\right)-\frac{4 y+1}{24 y} Y^{2}+
\end{aligned}
$$




$$
\begin{aligned}
& \left.+\frac{1}{8 y^{3}}\left(5 x^{2}+8 x-1\right) X-\frac{1}{8 y^{2} x}\left(21 x^{2}+35 x+12\right) Y+\frac{9 x+29}{24 y^{2}}\right], \\
L_{2}^{[3]}= & \frac{1}{72 y} X Y-\frac{x}{36 y} X^{2}-\frac{1}{36} Y^{2}+\frac{5 x}{54 y} X-\frac{\pi^{2}}{24 y}+\frac{5}{54} Y+ \\
& +\frac{i \pi}{72 y}\left((5+4 x) Y+(1-4 x) X-\frac{20}{3}\right) .
\end{aligned}
$$

For $h=3$ in eq. (2.24) and color factor $\operatorname{Tr}^{[1]}$ in eq. (2.31):

$$
\begin{aligned}
& A_{3}^{[1]}=\frac{1}{48 x y^{2}}\left(-3 x^{3}-31 x^{2}-2 x-4\right) X^{2}+ \\
& +\frac{1}{48 x y}\left(55 x y-16 y^{2}+60 x^{2}\right) X+\frac{175 y}{108 x}-\frac{25}{864}+ \\
& +i \pi\left(\frac{1}{24 x y^{2}}\left(-3 x^{3}-31 x^{2}-2 x-4\right) X+\frac{1}{48 x y}\left(55 x y-16 y^{2}+60 x^{2}\right)\right), \\
& B_{3}^{[1]}=-\frac{1}{16 y^{2}}\left(4 x^{3}+y^{3}+7 x^{2} y+3 x y^{2}\right) X^{2}- \\
& -\frac{y}{16 x^{2}}\left(6 x^{2}+6 y^{2}+11 x y\right) Y^{2}-\frac{1}{8}(1-y) Y X- \\
& -\frac{2}{24 y}(6-5 y) X-\frac{3(1-y)}{8 x} Y+\frac{\pi^{2}}{16}(1-y)-\frac{24}{72 x}-\frac{139}{72}+ \\
& +i \frac{\pi}{8}\left(-\frac{5 x+1}{y^{2}} X-\frac{7 y+1}{x^{2}} Y+\frac{1}{3 x y}\left(18 y^{2}+31 x y+12 x^{2}\right)\right), \\
& C_{3}^{[1]}=-\frac{y}{16 x^{2}}\left(6 x^{2}+6 y^{2}+11 x y\right) Y^{2}+\frac{y-1}{8} Y X-\frac{3 x-1}{16 y} X+ \\
& +\frac{1}{16 y^{2}}\left(6 x^{2} y+4 x y^{2}-y^{3}+2 x^{3}\right) X^{2}+(y-1)\left(\frac{3}{8 x} Y-\frac{\pi^{2}}{16}\right)-\frac{17}{32}+ \\
& +i \frac{\pi}{8}\left(-\frac{1}{y^{2}}\left(y^{2}+4 x y+2 x^{2}\right) X+\frac{6+7 x}{x^{2}} Y-\frac{1}{2 x y}\left(-12 y^{2}+4 x^{2}-5 x y\right)\right) \text {, } \\
& D_{3}^{[1]}=\frac{5 x^{2}+x+2}{24 x y^{2}} X^{2}+\frac{x^{2}+23 x+10}{24 x y} X-\frac{397 y}{216 x}-\frac{143}{108}+ \\
& +i \pi\left(\frac{5 x^{2}+x+2}{12 x y^{2}} X+\frac{x^{2}+23 x+10}{24 y x}\right) \text {, } \\
& E_{3}^{[1]}=-\frac{\pi^{2}}{8}(1+3 y)+\frac{1-y}{8 y^{2}}\left(3 x^{2}+2 x y+2 y^{2}\right) X^{2}+ \\
& +\frac{18 x+35 y}{24 y} X-\frac{y}{8 x^{2}}\left(2 y^{2}+6 x^{2}+5 x y\right) Y^{2}+ \\
& +\frac{1}{4}(1+3 y) Y X-\frac{3(1-y)}{4 x} Y+\frac{19 x+33}{72 x}- \\
& -i \pi\left(\frac{x-2}{4 y^{2}} X-\frac{1-y}{4 x^{2}} Y-\frac{1}{24 y x}\left(36 y^{2}+18 x^{2}+53 x y\right)\right), \\
& F_{3}^{[1]}=\frac{1}{12 x} X-\frac{5}{54 x}+i \frac{\pi}{12 x}, \\
& G_{3}^{[1]}=0 .
\end{aligned}
$$


For $h=3$ in eq. (2.24) and color factor $\operatorname{Tr}^{[2]}$ in eq. (2.31):

$$
\begin{aligned}
& A_{3}^{[2]}=\frac{1}{x y}\left[-\frac{1}{48}\left(30 x^{3}+39 x^{2}+10 x+4\right)\left((X-Y)^{2}+\pi^{2}\right)-\right. \\
& -\frac{1}{48}\left(60 x^{2}+55 x-16\right) X+\frac{1}{144}\left(180 x^{2}+121 x+40\right) Y+ \\
& \left.+\frac{25}{864}(x-56)\right]-i \pi \frac{11(x-2)}{36 x y}, \\
& B_{3}^{[2]}=\frac{6-x}{16 x^{2}} Y^{2}+\frac{1-y}{16 y^{2}} X^{2}+\frac{1}{16}(4 x-1)\left((X-Y)^{2}+\pi^{2}\right)- \\
& -\frac{6 x+11}{12 y} X+\frac{1}{8 x y}\left(4 x^{2}+3 x+6\right) Y+\frac{115 x-24}{72 x y}- \\
& -i \frac{\pi}{8}\left(\frac{x-6}{x^{2}} Y+\frac{y-1}{y^{2}} X+\frac{13 x-18}{3 x y}\right) \text {, } \\
& C_{3}^{[2]}=\frac{1}{16}\left[-\frac{x-6}{x^{2}} Y^{2}-\frac{y-1}{y^{2}} X^{2}+\frac{1}{y}\left(2 x^{2}+4 x+1\right)\left((X-Y)^{2}+\pi^{2}\right)+\right. \\
& \left.+\frac{4 x+1}{y} X-\frac{1}{x y}\left(4 x^{2}-5 x-12\right) Y+\frac{17}{2 y}\right]- \\
& -i \frac{\pi}{8}\left(\frac{x-6}{x^{2}} Y+\frac{y-1}{y^{2}} X+3 \frac{y-1}{x y}\right) \\
& D_{3}^{[2]}=\frac{1}{24 x y}\left[\left(12 x^{2}+3 x-10\right) X-\frac{1}{3}\left(36 x^{2}+49 x+22\right) Y+\frac{1}{9}(286 x+397)\right]- \\
& -\frac{1}{24 x}\left(6 x^{2}+3 x+2\right)\left((X-Y)^{2}+\pi^{2}\right)-i \pi \frac{10 x+13}{18 x y}, \\
& E_{3}^{[2]}=\frac{1}{8}\left[-\frac{x-2}{y^{2}} X^{2}-\frac{y-1}{x^{2}} Y^{2}+(3 x+2)\left((X-Y)^{2}+\pi^{2}\right)-\right. \\
& \left.-\frac{18 x+35}{3 y} X+\frac{1}{x y}\left(12+19 x+6 x^{2}\right) Y+\frac{33+14 x}{9 x y}\right]- \\
& -i \frac{\pi}{4}\left(\frac{x-2}{y^{2}} X+\frac{y-1}{x^{2}} Y-\frac{11 x+18}{3 x y}\right) \text {, } \\
& F_{3}^{[2]}=-\frac{1}{12 x}\left(X+\frac{1}{3} Y-\frac{10}{9}\right)-i \frac{\pi}{9 x}, \\
& G_{3}^{[2]}=0 \text {. }
\end{aligned}
$$

For $h=3$ in eq. (2.24) and color factor $\operatorname{Tr}^{[3]}$ in eq. (2.31):

$$
\begin{aligned}
H_{3}^{[3]}= & \frac{y-1}{8 x^{2} y}(1-x y) Y^{2}-\frac{1}{48 y^{2} x}\left(6 x^{3}+25 x^{2}-7 x+4\right) X^{2}-\frac{21 x+32}{18 x} Y- \\
& -\frac{1}{48 x y}\left(30 x^{3}+51 x^{2}+19 x+4\right)\left((X-Y)^{2}+\pi^{2}\right)+\frac{7(y-1)}{6 y} X+ \\
& +i \pi\left(\frac{y-1}{4 x^{2} y}(1-x y) Y-\frac{1}{24 y^{2} x}\left(6 x^{3}+25 x^{2}-7 x+4\right) X+\frac{11 x+32}{18 x y}\right), \\
I_{3}^{[3]}= & \frac{y-1}{8 x^{2} y}\left(x^{2}-2 x-2\right) Y^{2}-\frac{x-1}{16 y}\left((X-Y)^{2}+\pi^{2}\right)+
\end{aligned}
$$




$$
\begin{aligned}
& +\frac{x-y}{16 y} X^{2}-\frac{y-1}{12 y} X+\frac{x+12}{12 x} Y+ \\
& +i \pi\left(\frac{x-y}{8 y} X+\frac{y-1}{4 x^{2} y}\left(x^{2}-2 x-2\right) Y-\frac{11 x+12}{12 x y}\right), \\
J_{3}^{[3]}= & \frac{1}{24 x y^{2}}\left(5 x^{2}+x+2\right) X^{2}+\frac{y-1}{4 x^{2}} Y^{2}+\frac{39 x+94}{72 x} Y- \\
& -\frac{13(y-1)}{24 y} X-\frac{1}{24 x}\left(6 x^{2}+3 x+2\right)\left((X-Y)^{2}+\pi^{2}\right)+ \\
& +i \pi\left(\frac{y-1}{2 x^{2}} Y+\frac{1}{12 x y^{2}}\left(5 x^{2}+x+2\right) X-\frac{55 x+94}{72 x y}\right), \\
K_{3}^{[3]}= & \frac{x-2}{8 y^{2}} X^{2}+\frac{y-1}{8 x^{2}} Y^{2}-\frac{1}{8}(3 x+2)\left((X-Y)^{2}+\pi^{2}\right)+ \\
& +\frac{19 x+36}{24 x} Y-\frac{19(y-1)}{24 y} X+ \\
& +i \pi\left(\frac{y-1}{4 x^{2}} Y+\frac{x-2}{4 y^{2}} X-\frac{17 x+36}{24 x y}\right), \\
L_{3}^{[3]}= & -\frac{1}{36 x}(Y+i \pi) .
\end{aligned}
$$

For $h=4$ in eq. (2.25) and color factor $\operatorname{Tr}^{[1]}$ in eq. (2.31):

$$
\begin{aligned}
A_{4}^{[1]}=\frac{1}{2 y^{3}}\left[\frac{1}{2}\left(x^{3}+3 x^{2}+3 x-2\right)\left(\pi^{2} X^{2}-\operatorname{Li}_{4}(-x)\right)-X \operatorname{Li}_{3}(-x)+\right. \\
\quad+(1-x y) X^{2}\left(\frac{1}{4}(2+x) \operatorname{Li}_{2}(-x)+\frac{1}{8}(y-1) X^{2}\right)+ \\
\quad+\frac{1}{2}\left(15 x+5 x^{3}+15 x^{2}+7\right) \zeta_{3} X+\frac{13}{6} \pi^{2} X^{2}+ \\
\left.\quad+\frac{\pi^{4}}{480}(3 x+1)\left(19+24 x+9 x^{2}\right)\right]+ \\
+\frac{\pi^{2}}{6} \operatorname{Li}_{2}(-x)+\frac{\pi^{2}}{6} Y X-\frac{1}{12} Y X^{3}+ \\
+\frac{1}{288 y^{3}}\left[4\left(50+108 x+126 x^{2}+45 x^{3}\right) X^{3}-\right. \\
\left.\quad-\left(579 x+795 x^{2}+297 x^{3}+265\right) \pi^{2} X\right]+ \\
+\frac{1}{48 y^{2}}\left[\left(44+73 x+35 x^{2}\right)\left(2 X \operatorname{Li}_{2}(-x)+Y X^{2}-2 \mathrm{Li}_{3}(-x)\right)+\right. \\
\left.\quad+\left(241 x^{2}+470 x+205\right) \zeta_{3}\right]- \\
+\frac{1}{72 y^{2}}\left(18-73 x-45 x^{2}\right)-\frac{475}{288 y}-\frac{36077}{3456}+ \\
+\frac{1}{288 y^{2}}\left(18 x^{2}-431 x+501\right) X+ \\
\left.+\frac{\pi^{2}}{28}+505 x^{3}+368+918 x\right) X^{2}+
\end{aligned}
$$




$$
\begin{aligned}
& +i \frac{\pi}{4}\left[\frac { 1 } { y ^ { 3 } } \left((2+x)(1-x y) X \operatorname{Li}_{2}(-x)-(1-x y)(1-y) X^{3}-\right.\right. \\
& \left.-2 \operatorname{Li}_{3}(-x)+\frac{2 \pi^{2}}{3} X+\left(5 x^{3}+15 x^{2}+15 x+7\right) \zeta_{3}\right)- \\
& -Y X^{2}+\frac{1}{6 y^{3}}\left(50+108 x+126 x^{2}+45 x^{3}\right) X^{2}+ \\
& +\frac{1}{6 y^{2}}\left(44+73 x+35 x^{2}\right)\left(\operatorname{Li}_{2}(-x)+X Y\right)- \\
& -\frac{1}{36 y^{3}}\left(505 x^{3}+1530 x^{2}+368+918 x\right) X- \\
& \left.-\frac{\pi^{2}}{24 y^{2}}\left(21 x^{2}+50 x+45\right)+\frac{475}{36 y^{2}}+\frac{467}{72 y}+\frac{1}{4}\right] \text {, } \\
& B_{4}^{[1]}=\frac{1}{4 y^{3}}\left[(y-1)\left(4 x^{2}-5 x-5\right) \operatorname{Li}_{4}(-x)+\frac{x}{12}\left(x^{2}-3 x-3\right) X^{4}-\right. \\
& -2\left(4 x-4 x^{2}-6 x^{3}+1\right) X \operatorname{Li}_{3}(-x)- \\
& -\frac{\pi^{2}}{3} \operatorname{Li}_{2}(-x)\left(25 x^{3}+44 x^{2}+31 x+14\right)- \\
& -\left(11 x^{3}+17 x^{2}+13 x+9\right) \zeta_{3} X-\frac{\pi^{2}}{6}\left(4 x^{3}+3 x^{2}-6 x-12\right) X^{2}+ \\
& \left.+\frac{\pi^{4}}{720}\left(653 x^{3}-133 x^{2}-1241 x-343\right)\right]+ \\
& +\frac{1}{2 y^{2}}\left[-x(x+13) \operatorname{Li}_{4}(-y)-\left(7 x^{2}-x+4\right) \operatorname{Li}_{4}\left(-\frac{x}{y}\right)+\right. \\
& +\left(3 x+4+5 x^{2}\right)\left(\operatorname{Li}_{3}(-x)-\zeta_{3}\right) Y-2\left(2-x+3 x^{2}\right) X \operatorname{Li}_{3}(-y)+ \\
& +\frac{1}{4}\left(6 x^{2}-5 x-2\right) X^{2} \operatorname{Li}_{2}(-x)-2 \operatorname{Li}_{2}(-x)\left(2 x^{2}+x+2\right) X Y- \\
& -2 y Y^{2} \operatorname{Li}_{2}(-x)+4 y^{2} Y \operatorname{Li}_{3}(-y)-\frac{1}{4}\left(-3 x+13 x^{2}+8\right) X^{2} Y^{2}+ \\
& +\frac{1}{3}\left(5 x^{2}+4 x+5\right) Y^{3} X-\frac{1}{6}(1+7 x) Y X^{3}- \\
& -\frac{1}{24}\left(5 x^{2}-5 x+2\right) Y^{4}-\frac{\pi^{2}}{6}\left(8 x^{2}+9 x+7\right) Y^{2}+ \\
& \left.+\frac{\pi^{2}}{3}\left(6+4 x+13 x^{2}\right) Y X\right]+ \\
& +\frac{1}{y^{3}}\left[\frac{x}{4}\left(4+10 x+3 x^{2}\right)\left(X \operatorname{Li}_{2}(-x)-\operatorname{Li}_{3}(-x)\right)-\right. \\
& -\frac{x}{72}\left(6 x+32 x^{2}+15\right) X^{3}+ \\
& +\frac{1}{144}\left(1509 x+443 x^{3}+443+1617 x^{2}\right) \zeta_{3}+ \\
& \left.+\frac{\pi^{2}}{288}\left(477+891 x+999 x^{2}+733 x^{3}\right) X\right]+ \\
& +\frac{1}{24 y^{2}}\left(x(11 x-19) Y X^{2}-2\left(x^{2}+9 x+10\right) \pi^{2} Y\right)-
\end{aligned}
$$




$$
\begin{aligned}
& -\frac{1}{y}\left(3 x\left(\operatorname{Li}_{3}(-y)+Y \operatorname{Li}_{2}(-x)\right)+\frac{1}{12}(27 x+20) X Y^{2}\right)- \\
& -\frac{5}{6} Y^{3}-\frac{1}{144 y^{3}}\left(246 x^{2}-215 x^{3}+246 x+144\right) X^{2}- \\
& -\frac{1}{288 y^{2}}\left(4 x(145+91 x) X Y-\left(311 x^{2}-340 x-455\right) \pi^{2}\right)+ \\
& +\frac{1}{144 x}(191 x+27) Y^{2}-\frac{1}{216 y^{2}}\left(269 x^{2}+1966 x+782\right) X+ \\
& +\frac{3(x-y)}{8 y} Y-\frac{139}{72 y}+\frac{14135}{2592}+ \\
& +i \pi\left[\frac { 1 } { y ^ { 3 } } \left(\frac{1}{2}\left(x^{3}-4 x^{2}-11 x-5\right) \operatorname{Li}_{3}(-x)-\frac{\pi^{2}}{12}\left(-7 x^{2}+2 x^{3}-6-14 x\right) X+\right.\right. \\
& \left.+\frac{1}{4}\left(y^{3}+2 x^{2}+4 x\right) \zeta_{3}-\frac{1}{12} x(x y-2) X^{3}\right)+ \\
& +\frac{1}{y^{2}}\left(-(x-5) x \operatorname{Li}_{3}(-y)+\frac{1}{4}(2 x+5)(y-1) X \operatorname{Li}_{2}(-x)-\right. \\
& -x(2 x-1) Y \operatorname{Li}_{2}(-x)-\frac{\pi^{2}}{12} Y\left(17 x+3 x^{2}+8\right)- \\
& \left.-\frac{1}{2}\left(1-2 x+3 x^{2}\right) X Y^{2}\right)+ \\
& +\frac{1}{y}\left(-\frac{1}{12} x Y^{3}-\frac{1}{2} x X Y^{2}+\frac{1}{4}(3 x+1) X^{2} Y-\frac{\pi^{2}}{2} x X\right)- \\
& -\frac{x}{4 y^{3}}\left(9 x^{2}+14 x+8\right) \operatorname{Li}_{2}(-x)+\frac{\pi^{2}}{96 y^{3}}\left(23 x^{3}+93 x^{2}+161 x+79\right)- \\
& -\frac{x}{6 y^{3}}\left(13 x^{2}+7 x+2\right) X^{2}+\frac{1}{12 y^{2}}\left(29 x^{2}+39 x+40\right) X Y+ \\
& +\frac{21 x+10}{12 y} Y^{2}+\frac{1}{72 y^{3}}\left(306 x^{3}-10 x^{2}-101 x-144\right) X+ \\
& +\frac{1}{72 x y^{2}}\left(100 x^{3}+264 x^{2}+27+245 x\right) Y- \\
& \left.-\frac{1}{216 y^{2}}\left(431 x^{2}+2209 x+863\right)\right] \text {, } \\
& C_{4}^{[1]}=\frac{1}{2 y^{3}}\left[x ( x ^ { 2 } - 4 x + 1 ) \left(\operatorname{Li}_{4}(-y)-\operatorname{Li}_{4}\left(-\frac{x}{y}\right)+\left(\operatorname{Li}_{3}(-x)-\zeta_{3}\right) Y-\right.\right. \\
& \left.-\frac{1}{24} Y^{4}-\frac{\pi^{2}}{12} Y^{2}+\frac{1}{6} Y^{3} X\right)+ \\
& +\frac{1}{2} x\left(5 x^{2}+8 x-2\right) \operatorname{Li}_{4}(-x)-2 x^{3} X \operatorname{Li}_{3}(-x)+ \\
& +\frac{x^{3}}{4} \operatorname{Li}_{2}(-x) X^{2}+\frac{\pi^{2}}{6} x\left(x^{2}+4 x-1\right) \operatorname{Li}_{2}(-x)-\frac{x^{3}}{12} X^{4}- \\
& -\left(x^{3}+9 x^{2}+9 x+3\right) \zeta_{3} X+\frac{\pi^{2}}{12} x^{3} X^{2}- \\
& \left.-\frac{\pi^{4}}{360}\left(16 x^{3}-5 x^{2}-40 x-11\right)\right]+
\end{aligned}
$$




$$
\begin{aligned}
& +\frac{1}{24 y^{3}}\left[9 x\left(5 x^{2}+1+4 x\right)\left(\operatorname{Li}_{2}(-x) X-\operatorname{Li}_{3}(-x)\right)+\right. \\
& +3\left(15+29 x+21 x^{3}+29 x^{2}\right) \zeta_{3}+x\left(1+4 x+9 x^{2}\right) X^{3}+ \\
& \left.+\left(3+10 x+13 x^{2}+9 x^{3}\right) \pi^{2} X\right]+ \\
& +\frac{1}{4 y^{2}}\left[3 x(x-1)\left(2 \operatorname{Li}_{3}(-y)+2 \operatorname{Li}_{2}(-x) Y+X Y^{2}\right)-\right. \\
& \left.-\frac{1}{12} x(3 x+1) Y\left(3 X^{2}+4 \pi^{2}\right)\right]- \\
& -\frac{x}{32 y^{3}}\left(17 x^{2}+6+34 x\right) X^{2}-\frac{1}{16 y x}\left(2 x+11 x^{2}+3\right) Y^{2}- \\
& -\frac{1}{96 y^{2}}\left(12 x(5+11 x) X Y-\left(51 x^{2}+100 x+29\right) \pi^{2}\right)+ \\
& +\frac{1}{32 y^{2}}\left(66 x^{2}+23 x+3\right) X+\frac{3(x-y)}{8 y} Y-\frac{17}{32 y}+\frac{187}{128}+ \\
& +i \pi\left[\frac { 1 } { 1 2 y ^ { 3 } } \left(6 x\left(1-4 x-x^{2}\right)\left(\operatorname{Li}_{3}(-x)-\zeta_{3}\right)+\right.\right. \\
& \left.+3 x^{3} \operatorname{Li}_{2}(-x) X+x^{3} \pi^{2} X-x^{3} X^{3}\right)+\frac{3}{2} \zeta_{3}+ \\
& +\frac{1}{24 y^{3}}\left(9 x\left(x^{2}+4 x+5\right) \operatorname{Li}_{2}(-x)+9 x^{3} X^{2}-\left(3 x^{3}-5 x^{2}-8 x-3\right) \pi^{2}\right)- \\
& -\frac{x}{8 y^{2}}(3 x+1) X Y+\frac{x}{16 y^{3}}\left(5 x^{2}+4-2 x\right) X+ \\
& \left.+\frac{1}{8 x y^{2}}\left(8 x^{2}+5 x+3\right) Y+\frac{1}{32 y^{2}}(3 x+1)(14 x-9)\right] \text {, } \\
& D_{4}^{[1]}=-\frac{1}{144 y^{3}}\left[2\left(9 x^{3}+27 x+27 x^{2}+14\right) X^{3}-\left(47+81 x+27 x^{3}+81 x^{2}\right) \pi^{2} X\right]+ \\
& +\frac{1}{3}\left(\operatorname{Li}_{3}(-x)-\mathrm{Li}_{2}(-x) X\right)-\frac{17}{24} \zeta_{3}-\frac{1}{6} Y X^{2}+ \\
& +\frac{1}{72 y^{3}}\left(43 x^{3}+90 x+135 x^{2}+35\right) X^{2}+\frac{\pi^{2}}{216 y^{2}}\left(77 x^{2}+139 x+32\right)+ \\
& +\frac{1}{216 y^{2}}\left(53+400 x+125 x^{2}\right) X+\frac{37}{72 y}+\frac{1307}{864}+ \\
& +i \pi\left[-\frac{1}{3} \operatorname{Li}_{2}(-x)-\frac{1}{3} X Y-\frac{1}{24 y^{3}}\left(9 x^{3}+27 x+27 x^{2}+14\right) X^{2}+\right. \\
& +\frac{1}{16} \pi^{2}+\frac{1}{36 y^{3}}\left(43 x^{3}+90 x+135 x^{2}+35\right) X+ \\
& \left.+\frac{1}{216 y^{2}}\left(125 x^{2}+400 x+53\right)\right] \text {, } \\
& E_{4}^{[1]}=\frac{x(x-1)}{y^{3}}\left[-2\left(\operatorname{Li}_{4}(-x)+\operatorname{Li}_{4}(-y)-\operatorname{Li}_{4}\left(-\frac{x}{y}\right)\right)+\right. \\
& +2(X-Y)\left(\operatorname{Li}_{3}(-x)-\zeta_{3}\right)-\frac{\pi^{2}}{3} \operatorname{Li}_{2}(-x)-
\end{aligned}
$$




$$
\begin{aligned}
& \left.-\frac{1}{3} X Y^{3}+\frac{1}{12} Y^{4}+\frac{1}{12} X^{4}+\frac{\pi^{2}}{6} Y^{2}+\frac{\pi^{2}}{3} X^{2}+\frac{7}{60} \pi^{4}\right]+ \\
& +\frac{1}{144 y^{3}}\left[48\left(15 x+15 x^{2}+x^{3}+2\right)\left(\operatorname{Li}_{3}(-x)-\operatorname{Li}_{2}(-x) X\right)+\right. \\
& +2 x\left(13 x^{2}-24-24 x\right) X^{3}-2\left(x^{3}+3 x^{2}+25+3 x\right) \zeta_{3}- \\
& \left.-3 \pi^{2}\left(13 x^{3}+9+71 x+71 x^{2}\right) X\right]+ \\
& +\frac{1}{6 y^{2}}\left[-2\left(x^{2}+14 x+1\right)\left(\operatorname{Li}_{3}(-y)+Y \operatorname{Li}_{2}(-x)\right)+\frac{1}{4}\left(6 x^{2}+17 x+8\right) Y X^{2}-\right. \\
& \left.-\left(2 x^{2}+17 x+3\right) X Y^{2}-\frac{\pi^{2}}{6}\left(2 x^{2}-53 x-7\right) Y\right]+ \\
& +\frac{1}{12} Y^{3}-\frac{x}{72 y^{3}}\left(123+312 x+244 x^{2}+54 x^{3}\right) X^{2}+ \\
& +\frac{\pi^{2}}{144 y^{2}}\left(108 x^{3}+287 x^{2}+128 x+33\right)-\frac{1}{36 y}\left(44 x+27 x^{2}-19\right) Y^{2}- \\
& -\frac{x}{18 y^{2}}\left(43+76 x+27 x^{2}\right) X Y+\frac{1}{216 y^{2}}\left(353 x^{2}+478 x+191\right) X+ \\
& +\frac{9 x+4}{6 y} Y+\frac{19}{72 y}-\frac{3401}{2592}+ \\
& +i \pi\left[\frac { 1 } { 3 y ^ { 3 } } \left(\frac{\pi^{2}}{48}\left(21 x^{3}+51 x^{2}+39 x+17\right)-\mathrm{Li}_{2}(-x)+\right.\right. \\
& \left.+\frac{x}{8}\left(13 x+11 x^{2}+11\right) X^{2}\right)+ \\
& +\frac{x(2 x+5)}{12 y^{2}} X Y-\frac{(x-1)}{12 y} Y^{2}-\frac{x}{36 y^{3}}\left(38 x^{2}+37+74 x\right) X- \\
& \left.-\frac{1}{18 y^{2}}\left(18 x+5 x^{2}+19\right) Y-\frac{8 x-3}{36 y^{2}}+\frac{29}{216}\right] \text {, } \\
& F_{4}^{[1]}=\frac{1}{24} X^{2}-\frac{\pi^{2}}{27}-\frac{5}{54} X+i \pi\left(\frac{1}{12} X-\frac{5}{54}\right), \\
& G_{4}^{[1]}=\frac{1}{72 y}\left(x Y X+Y^{2} y+\pi^{2}\right)+i \frac{\pi}{72 y}(x X+(y-1) Y) .
\end{aligned}
$$

For $h=4$ in eq. (2.25) and color factor $\operatorname{Tr}^{[2]}$ in eq. (2.31):

$$
\begin{aligned}
A_{4}^{[2]}=\frac{1}{4 y^{3}}[ & -4(x-4) x\left(\operatorname{Li}_{4}(-x)+\operatorname{Li}_{4}(-y)\right)- \\
& -\left(3 x^{3}+4 x^{2}+23 x+2\right)\left(\operatorname{Li}_{4}\left(-\frac{x}{y}\right)-\frac{\pi^{2}}{6} \operatorname{Li}_{2}(-x)\right)- \\
& -2\left(x^{3}+x^{2}+11 x+1\right)(X-Y) \operatorname{Li}_{3}(-x)+ \\
& +\frac{1}{12}\left(3 x^{3}+14 x^{2}+7 x+6\right) X^{4}+\frac{1}{6}\left(x^{3}+5 x^{2}+x+2\right) Y^{4}- \\
& -\frac{1}{6}\left(x^{3}+10 x^{2}-7 x+4\right)\left(X Y^{3}-\pi^{2} X^{2}\right)+ \\
& +\frac{\pi^{2}}{12}(x-1)\left(x^{2}+9 x-2\right) Y^{2}-4(6 x+1) \zeta_{3} Y-
\end{aligned}
$$




$$
\begin{aligned}
& \left.-\left(9 x^{2}-6 x+5\right) \zeta_{3} X-\frac{\pi^{4}}{240}\left(12 x^{3}-53 x^{2}+194 x-21\right)\right]- \\
& -\frac{y-1}{4 y}\left(\frac{1}{2}(X-Y)^{2} \operatorname{Li}_{2}(-x)+\frac{3}{4} X^{2} Y^{2}\right)- \\
& -\frac{1}{y}\left(\frac{1}{12}(3 x+5) X^{3} Y+\frac{\pi^{2}}{6} X Y\right)+\frac{1}{2}(X-Y) \operatorname{Li}_{3}(-y)- \\
& -\frac{1}{6 y^{2}}\left[\left(9 x^{2}+50 x+11\right)\left(\operatorname{Li}_{3}(-x)+\operatorname{Li}_{3}(-y)-X \operatorname{Li}_{2}(-x)+Y \operatorname{Li}_{2}(-x)\right)-\right. \\
& -\frac{1}{6}\left(16 x^{2}+71 x+25\right) X^{3}+\frac{1}{2}\left(24 x^{2}+95 x+41\right) X Y^{2}- \\
& -\frac{\pi^{2}}{24}\left(176 x^{2}+683 x+267\right) Y- \\
& \left.-\frac{5}{48} \pi^{2}\left(40 x^{2}+203 x+67\right) X-\frac{117}{8} y \zeta_{3}\right]+ \\
& +\frac{y-1}{3 y}\left(X^{2} Y+\frac{13}{6} Y^{3}\right)+\frac{\pi^{2}}{288 y^{2}}\left(360 x^{3}+1073 x^{2}+1001 x+528\right)- \\
& -\frac{1}{288 y}\left(x(360 x+713)(X-Y)^{2}-368 X^{2}+560 X Y-1160 Y^{2}\right)+ \\
& +\frac{1}{y}\left(\frac{1}{54}(135 x+2) Y-\frac{1}{96}(240 x-167) X\right)+\frac{30593 x+30377}{3456 y^{2}}+ \\
& +i \pi\left[\frac{11(y-1)}{12 y}\left((X-Y)^{2}+\pi^{2}\right)-\frac{9}{4 y} \zeta_{3}+\frac{11 \pi^{2}}{288 y}+\right. \\
& \left.+\frac{11}{18 y}(X+10 Y)+\frac{1535}{864 y}\right] \\
& B_{4}^{[2]}=\frac{1}{2 y^{3}}\left[\left(3 x^{3}-12 x-4\right) \operatorname{Li}_{4}(-x)-\left(x^{2}+22 x+14\right) x \operatorname{Li}_{4}(-y)-\right. \\
& -\left(7 x^{3}-x^{2}+4 x+5\right) \operatorname{Li}_{4}\left(-\frac{x}{y}\right)-\left(7 x^{3}+x^{2}-4 x+1\right) X \operatorname{Li}_{3}(-x)+ \\
& +\left(6 x^{3}+3 x^{2}+9 x+5\right) Y \operatorname{Li}_{3}(-x)+\frac{\pi^{2}}{6}\left(23 x^{3}+39 x^{2}+24 x+13\right) \operatorname{Li}_{2}(-x)+ \\
& +\frac{x}{24}\left(x^{2}+4 x+2\right) X^{4}-\frac{1}{24}(3 x+2)\left(x^{2}-4 x+2\right) Y^{4}+ \\
& +\frac{1}{6}\left(8 x^{3}+10 x^{2}+23 x+14\right) X Y^{3}-\frac{\pi^{2}}{12}\left(x^{3}-17 x^{2}-4 x+12\right) X^{2}- \\
& -\frac{\pi^{2}}{12}\left(15 x^{3}+27 x^{2}+36 x+17\right) Y^{2}-\left(6 x^{3}+3 x^{2}+9 x+5\right) \zeta_{3} Y+ \\
& \left.+\frac{1}{2}\left(12 x^{3}+7 x^{2}+8 x+11\right) \zeta_{3} X-\frac{\pi^{4}}{1440}\left(592 x^{3}-551 x^{2}-1570 x-343\right)\right]+ \\
& +\frac{1}{2 y^{2}}\left[\left(5 x^{2}-2 x+5\right) X \operatorname{Li}_{3}(-y)-\frac{1}{2}\left(4 x^{2}-x+1\right) X^{2} \operatorname{Li}_{2}(-x)+\right. \\
& +\left(4 x^{2}+3 x+5\right) X Y \operatorname{Li}_{2}(-x)+\frac{1}{6}(5 x-1) X^{3} Y+
\end{aligned}
$$




$$
\begin{aligned}
& \left.+\frac{1}{4}\left(10 x^{2}-3 x+11\right) X^{2} Y^{2}-\frac{\pi^{2}}{2}\left(7 x^{2}+x+4\right) X Y\right]+ \\
& +\frac{1}{2 y}\left((4 x+5) Y \operatorname{Li}_{3}(-y)+\frac{5}{2} Y^{2} \operatorname{Li}_{2}(-x)\right)+ \\
& +\frac{1}{4 y^{3}}\left[-x\left(6 x^{2}+1\right)\left(\operatorname{Li}_{3}(-x)-X \operatorname{Li}_{2}(-x)\right)-\right. \\
& -\frac{x}{18}\left(14 x^{2}+40 x+29\right) X^{3}+\frac{x}{2}\left(4 x^{2}-3\right) X^{2} Y- \\
& -\frac{\pi^{2}}{72}\left(176 x^{3}+853 x^{2}+782 x+477\right) X- \\
& \left.-\frac{1}{36}\left(1235 x^{2}+1426 x+443\right) \zeta_{3}\right]+ \\
& +\frac{1}{2 y^{2}}\left[-(3 x+10) x\left(\operatorname{Li}_{3}(-y)+\operatorname{Li}_{2}(-x) Y\right)-\right. \\
& \left.-\frac{1}{6}\left(33 x^{2}+74 x+20\right) X Y^{2}+\frac{\pi^{2}}{9}\left(10 x^{2}+28 x+15\right) Y\right]- \\
& -\frac{1}{144 y^{3}}\left(72 x^{4}+145 x^{3}-304 x^{2}-422 x-144\right) X^{2}- \\
& -\frac{28 x+15}{18 y} Y^{3}-\frac{1}{8 y^{2}}\left(8 x^{3}-3 x^{2}-39 x-16\right) X Y+ \\
& +\frac{\pi^{2}}{288 y^{2}}\left(144 x^{3}+146 x^{2}-83 x+167\right)+\frac{1}{216 y^{2}}\left(216 x^{2}+917 x+782\right) X- \\
& -\frac{1}{144 x y}\left(72 x^{3}-199 x^{2}-290 x+27\right) Y^{2}+\frac{24 x-71}{24 y} Y-\frac{18815 x+19139}{2592 y^{2}}+ \\
& +i \pi\left[\frac{1}{y^{3}}\left(-\frac{1}{2}\left(x^{3}-2 x^{2}-13 x-4\right) \operatorname{Li}_{3}(-x)-3 \zeta_{3} x\right)+\right. \\
& +\frac{1}{2 y^{2}}\left((x-11) x \operatorname{Li}_{3}(-y)+2(2 x-1) x Y \operatorname{Li}_{2}(-x)+x X^{3}+\right. \\
& \left.+\left(3 x^{2}-2 x+1\right) X Y^{2}+\frac{\pi^{2}}{2}(3 x+2) X-\frac{\pi^{2}}{6}\left(3 x^{2}-11 x-8\right) Y\right)+ \\
& +\frac{1}{y}\left(-2 X \operatorname{Li}_{2}(-x)-\frac{x}{4} X^{2} Y+\frac{1}{2} x X Y^{2}+\frac{x}{12} Y^{3}+\frac{\pi^{2}}{2} x(X-Y)+\frac{1}{4} \zeta_{3}\right)+ \\
& +\frac{1}{4 y^{3}}\left(\left(12 x^{2}+26 x+21\right) x \operatorname{Li}_{2}(-x)-\frac{x}{6}\left(20 x^{2}+40 x+41\right) X^{2}+\right. \\
& +\frac{1}{3}\left(60 x^{3}+136 x^{2}+119 x+40\right) X Y- \\
& \left.-\frac{\pi^{2}}{24}\left(112 x^{3}+79+258 x+263 x^{2}\right)\right)- \\
& -\frac{16 x+5}{6 y} Y^{2}-\frac{x}{72 y^{3}}\left(100 x^{2}+74 x+73\right) X- \\
& \left.-\frac{1}{72 x y^{2}}\left(100 x^{3}+138 x^{2}+119 x-27\right) Y+\frac{1}{216 y^{2}}(1340 x+1421)\right] \text {, }
\end{aligned}
$$




$$
\begin{aligned}
& C_{4}^{[2]}=\frac{1}{2 y^{3}}\left[\frac{x}{2}\left(5 x^{2}+14 x+7\right)\left(\operatorname{Li}_{4}\left(-\frac{x}{y}\right)-\frac{\pi^{2}}{6} \operatorname{Li}_{2}(-x)-\frac{1}{6} X Y^{3}\right)-\right. \\
& -\left(x^{2}+4 x+2\right) x\left(\operatorname{Li}_{4}(-y)+\mathrm{Li}_{4}(-x)+Y \mathrm{Li}_{3}(-x)\right)+ \\
& +\left(2 x^{2}+6 x+3\right) x X \operatorname{Li}_{3}(-x)-\frac{x}{24}\left(2 x^{2}+2 x+1\right) X^{4}+ \\
& +\frac{x}{24}\left(x^{2}+4 x+2\right) Y^{4}+\frac{\pi^{2}}{24} x\left(3 x^{2}+10 x+5\right) Y^{2}+ \\
& +\frac{\pi^{2}}{12} x\left(x^{2}+6 x+3\right) X^{2}+\left(x^{2}+5 x+3\right) \zeta_{3} X+ \\
& \left.+(x-y) x \zeta_{3} Y+\frac{\pi^{4}}{360}\left(41 x^{3}+113 x^{2}+40 x-11\right)\right]+ \\
& +\frac{x}{2 y}\left[(2 X-Y) \operatorname{Li}_{3}(-y)-\frac{1}{4}(X-Y)^{2} \operatorname{Li}_{2}(-x)+\right. \\
& \left.+\frac{1}{3} X^{3} Y+\frac{3}{8} X^{2} Y^{2}-\frac{\pi^{2}}{2} X Y\right]+ \\
& +\frac{1}{y^{3}}\left[-\frac{x}{4}\left(6 x^{2}+12 x+7\right)\left(\operatorname{Li}_{3}(-x)-X \operatorname{Li}_{2}(-x)\right)+\right. \\
& +\frac{x}{24}\left(6 x^{2}+8 x+1\right) X^{3}-\frac{1}{8}\left(11 x^{2}+24 x+15\right) \zeta_{3}+ \\
& \left.+\frac{x}{8}(2 x+3) X^{2} Y+\frac{\pi^{2}}{24}\left(12 x^{3}+19 x^{2}+5 x-3\right) X\right]+ \\
& +\frac{x(3 x+2)}{2 y^{2}}\left(\operatorname{Li}_{3}(-y)+Y \operatorname{Li}_{2}(-x)-\frac{\pi^{2}}{3} Y+\frac{1}{2} X Y^{2}\right)+ \\
& +\frac{x}{32 y^{3}}\left(8 x^{3}+31 x^{2}+42 x+21\right) X^{2}-\frac{x(8 x+15)}{16 y} X Y- \\
& -\frac{\pi^{2}}{96 y^{2}}\left(24 x^{3}+69 x^{2}+86 x+29\right)+\frac{1}{32 x y}\left(8 x^{3}+15 x^{2}-12 x-6\right) Y^{2}- \\
& -\frac{1}{32 y^{2}}\left(16 x^{2}+31 x+3\right) X-\frac{4 x-3}{8 y} Y-\frac{247 x+255}{128 y^{2}}+ \\
& +i \pi\left[\frac{x}{2 y}\left(\operatorname{Li}_{3}(-x)+\operatorname{Li}_{3}(-y)-\frac{1}{6} X^{3}+\frac{1}{2} X^{2} Y-\frac{\pi^{2}}{6} X\right)+\frac{3}{2 y} \zeta_{3}+\right. \\
& +\frac{x(2 x+3)}{24 y^{3}}\left(6 \mathrm{Li}_{2}(-x)+6 X Y-3 X^{2}-\pi^{2}\right)-\frac{\pi^{2}}{8 y}+ \\
& \left.+\frac{x(2 x+3)}{8 y^{3}} X-\frac{3(x-y)}{8 x y} Y-\frac{3}{32 y^{2}}(9 x+5)\right], \\
& D_{4}^{[2]}=\frac{x(x-1)}{y^{3}}\left[\operatorname{Li}_{4}(-x)+\operatorname{Li}_{4}(-y)-\mathrm{Li}_{4}\left(-\frac{x}{y}\right)+X\left(-\mathrm{Li}_{3}(-x)+\zeta_{3}\right)+\right. \\
& +Y\left(\operatorname{Li}_{3}(-x)-\zeta_{3}\right)+\frac{\pi^{2}}{6} \operatorname{Li}_{2}(-x)-\frac{1}{24} X^{4}+\frac{1}{6} X Y^{3}- \\
& \left.-\frac{1}{24} Y^{4}-\frac{\pi^{2}}{6} X^{2}-\frac{\pi^{2}}{12} Y^{2}-\frac{7}{120} \pi^{4}\right]+ \\
& +\frac{1}{3 y^{2}}\left[(7 x+1)\left(\operatorname{Li}_{3}(-x)+\operatorname{Li}_{3}(-y)+(Y-X) \operatorname{Li}_{2}(-x)\right)-\right.
\end{aligned}
$$




$$
\begin{aligned}
& -\frac{1}{24}\left(5 x^{2}+43 x+14\right) X^{3}+\frac{1}{8}\left(3 x^{2}+37 x+10\right) X Y^{2}- \\
& \left.-\frac{\pi^{2}}{24}\left(7 x^{2}+76 x+21\right) Y-\frac{\pi^{2}}{48}\left(10 x^{2}+143 x+37\right) X\right]+ \\
& +\frac{1}{8 y}\left(\frac{1}{3}(y-1) X^{2} Y-\frac{7}{9}(y-1) Y^{3}-3 \zeta_{3}\right)- \\
& -\frac{\pi^{2}}{216 y^{2}}\left(108 x^{3}+321 x^{2}+332 x+191\right)+ \\
& +\frac{1}{72 y}\left(x(36 x+71)(X-Y)^{2}-35 X^{2}+18 X Y-71 Y^{2}\right)+ \\
& +\frac{216 x+53}{216 y} X-\frac{9 x-7}{9 y} Y+\frac{863}{864 y}+ \\
& +i \pi\left[\frac{1-y}{6 y}\left((Y-X)^{2}+\pi^{2}\right)-\frac{\pi^{2}}{144 y}-\frac{13}{18 y} X-\frac{31}{18 y} Y+\frac{221}{216 y}\right], \\
& E_{4}^{[2]}=\frac{x(x-1)}{y^{3}}\left[-2\left(\operatorname{Li}_{4}(-x)-\operatorname{Li}_{4}\left(-\frac{x}{y}\right)+\operatorname{Li}_{4}(-y)\right)-\frac{\pi^{2}}{3} \operatorname{Li}_{2}(-x)+\right. \\
& +2(X-Y)\left(\operatorname{Li}_{3}(-x)-\zeta_{3}\right)+\frac{1}{12}\left(X^{4}+Y^{4}\right)- \\
& \left.-\frac{1}{3} X Y^{3}+\frac{\pi^{2}}{6}\left(Y^{2}+2 X^{2}\right)+\frac{7}{60} \pi^{4}\right]+ \\
& +\frac{1}{3 y^{3}}\left[\left(x^{3}+15 x^{2}+15 x+2\right)\left(\operatorname{Li}_{3}(-x)-X \operatorname{Li}_{2}(-x)\right)+\right. \\
& +\frac{\pi^{2}}{48}\left(42 x^{3}-109 x^{2}-116 x+27\right) X+ \\
& \left.+\frac{x}{24}\left(13 x^{2}-14 x-19\right) X^{3}+\frac{1}{24}\left(x^{2}+2 x-23\right) \zeta_{3}\right]+ \\
& +\frac{1}{3 y^{2}}\left[-\left(x^{2}+14 x+1\right)\left(\operatorname{Li}_{3}(-y)+Y \operatorname{Li}_{2}(-x)\right)+\right. \\
& +\frac{1}{8}\left(11 x^{2}+16 x+8\right) X^{2} Y-\frac{1}{8}\left(5 x^{2}+57 x+4\right) X Y^{2}+ \\
& \left.+\frac{\pi^{2}}{24}\left(13 x^{2}+111 x+2\right) Y\right]+ \\
& +\frac{13 x+6}{72 y} Y^{3}-\frac{x}{72 y^{3}}\left(54 x^{3}+235 x^{2}+338 x+139\right) X^{2}- \\
& -\frac{x}{36 y^{2}}\left(54 x^{2}+191 x+125\right) X Y-\frac{1}{72 y}\left(54 x^{2}+147 x+2\right) Y^{2}+ \\
& +\frac{\pi^{2}}{144 y^{2}}\left(108 x^{3}+362 x^{2}+117 x-33\right)+\frac{3 x+2}{2 y} Y+ \\
& +\frac{1}{216 y^{2}}\left(324 x^{2}-47 x-191\right) X-\frac{4085}{2592 y}+ \\
& +i \pi\left[\frac { 1 } { 3 y ^ { 3 } } \left(-\mathrm{Li}_{2}(-x)+\frac{x}{8}\left(6 x^{2}+19 x+17\right) X^{2}+\right.\right. \\
& \left.+\frac{\pi^{2}}{48}\left(48 x^{3}+135 x^{2}+126 x+47\right)\right)+\frac{7 x+3}{12 y} Y^{2}+
\end{aligned}
$$




$$
\begin{aligned}
& +\frac{1}{12 y^{2}}\left(10 x^{2}+15 x+8\right) X Y+\frac{x}{18 y^{3}}\left(5 x^{2}-11 x-7\right) X+ \\
& \left.+\frac{1}{18 y^{2}}\left(5 x^{2}+12 x+1\right) Y-\frac{587 x+407}{216 y^{2}}\right], \\
F_{4}^{[2]}= & \frac{1}{y}\left(\frac{1}{24}\left(X^{2}+Y^{2}\right)+\frac{1}{36} X Y-\frac{5}{54}(X+Y)-\frac{23}{216} \pi^{2}\right)+ \\
& +i \frac{\pi}{9 y}\left((X+Y)-\frac{5}{3}\right), \\
G_{4}^{[2]}= & -G_{4}^{[1]} .
\end{aligned}
$$

For $h=4$ in eq. (2.25) and color factor $\operatorname{Tr}^{[3]}$ in eq. (2.31):

$$
\begin{aligned}
& H_{4}^{[3]}=\frac{1}{2 y^{3}}\left[-\frac{1}{2}\left(16 x^{3}+47 x^{2}-23 x-10\right) \operatorname{Li}_{4}(-x)-\right. \\
& -\left(13 x^{3}+31 x^{2}+56 x+5\right) \operatorname{Li}_{4}\left(-\frac{x}{y}\right)-\left(8 x^{3}+32 x^{2}+7 x+16\right) \operatorname{Li}_{4}(-y)- \\
& -\left(10 x^{3}+25 x^{2}+20 x+1\right) X \operatorname{Li}_{3}(-y)-\left(x^{3}-x^{2}+28 x+2\right) X \operatorname{Li}_{3}(-x)+ \\
& +\left(8 x^{3}+15 x^{2}+39 x+1\right) Y \operatorname{Li}_{3}(-x)-\left(5 x^{3}+9 x^{2}+3 x-3\right) X Y \operatorname{Li}_{2}(-x)- \\
& -\frac{1}{4}(2 x+3)\left(3 x^{2}+5 x+4\right) X^{2} \operatorname{Li}_{2}(-x)-\frac{x}{24}\left(4 x^{2}-11 x+11\right) X^{4}+ \\
& +\frac{\pi^{2}}{6}\left(25 x^{3}+53 x^{2}+70 x+11\right) \operatorname{Li}_{2}(-x)- \\
& -\frac{1}{4}\left(13 x^{3}+21 x^{2}+3 x-13\right) X^{2} Y^{2}-\frac{1}{6}\left(9 x^{3}+38 x^{2}+49 x+22\right) X^{3} Y+ \\
& +\frac{1}{6}\left(34 x^{3}+86 x^{2}+103 x+18\right) X Y^{3}-\frac{1}{24}\left(10 x^{3}+22 x^{2}+47 x+2\right) Y^{4}+ \\
& +\frac{\pi^{2}}{12}(x+3)\left(14 x^{2}-13 x+4\right) X^{2}+\frac{\pi^{2}}{3}\left(4 x^{3}+8 x^{2}+4 x-5\right) X Y- \\
& -\frac{\pi^{2}}{12}\left(7 x^{3}+9 x^{2}+30 x-5\right) Y^{2}-\left(9 x^{3}+18 x^{2}+42 x+2\right) \zeta_{3} Y+ \\
& \left.+\left(9 x^{3}+14 x^{2}+34 x+1\right) \zeta_{3} X+\frac{\pi^{4}}{360}\left(10 x^{3}+334 x^{2}-121 x+184\right)\right]+ \\
& +\frac{1}{4 y}\left(2(15 x+13) Y \operatorname{Li}_{3}(-y)+(9 x+5) Y^{2} \operatorname{Li}_{2}(-x)+2(1-y)\left(X^{2}-Y^{2}\right) \pi^{2}\right)+ \\
& +\frac{1}{12 y^{3}}\left[\left(53 x^{3}+352 x^{2}+392 x+124\right)\left(\operatorname{Li}_{3}(-x)-X \operatorname{Li}_{2}(-x)\right)-\right. \\
& -\left(19 x^{3}+43 x^{2}+47 x+19\right) X^{2} Y+ \\
& +\frac{x}{3}\left(68 x^{2}-7 x-35\right) X^{3}-\left(52 x^{2}+83 x+62\right) \zeta_{3}- \\
& \left.-\frac{\pi^{2}}{12}\left(585 x^{3}+2038 x^{2}+2147 x+780\right) X\right]+ \\
& +\frac{1}{12 y^{2}}\left[-\left(27 x^{2}+263 x+62\right)\left(\operatorname{Li}_{3}(-y)+Y \operatorname{Li}_{2}(-x)\right)-\right. \\
& \left.-\left(4 x^{2}+132 x+41\right) X Y^{2}-\frac{\pi^{2}}{12}\left(309 x^{2}-646 x-307\right) Y\right]+
\end{aligned}
$$




$$
\begin{aligned}
& +\frac{11}{18} Y^{3}-\frac{x}{72 y^{3}}\left(108 x^{3}+949 x^{2}+1193 x+487\right) X^{2}- \\
& -\frac{1}{12 y^{2}}\left(36 x^{3}+185 x^{2}+108 x+10\right) X Y+ \\
& +\frac{\pi^{2}}{72 y^{2}}\left(108 x^{3}+450 x^{2}+363 x+250\right)+\frac{1}{108 y}(211 x+76) Y- \\
& -\frac{1}{72 y}\left(108 x^{2}+161 x-208\right) Y^{2}+\frac{x}{108 y^{2}}(211 x+121) X+ \\
& +i \pi\left[\frac { 1 } { 2 y ^ { 3 } } \left(\left(7 x^{3}+16 x^{2}+11 x-1\right) \operatorname{Li}_{3}(-x)+\right.\right. \\
& +\left(5 x^{3}+18 x^{2}+21 x+12\right) \operatorname{Li}_{3}(-y)- \\
& -\frac{1}{2}\left(16 x^{3}+37 x^{2}+29 x+6\right) X \operatorname{Li}_{2}(-x)- \\
& -2(y-1)\left(2 x^{2}+3 x+2\right) Y \operatorname{Li}_{2}(-x)- \\
& -\frac{\pi^{2}}{3}\left(8 x^{3}+28 x^{2}+32 x+13\right) Y+ \\
& +\frac{1}{2}\left(9 x^{3}+36 x^{2}+45 x+22\right) X Y^{2}- \\
& \left.-\frac{1}{6}\left(6 x^{3}+9 x^{2}+9 x+8\right) X^{3}-\left(4 x^{2}+8 x+1\right) \zeta_{3}\right)- \\
& -\frac{\pi^{2}}{4 y^{2}}\left(2 x^{2}+3 x+2\right) X-\frac{1}{y}\left(\frac{1}{4}(7 x+11) X^{2} Y+\frac{1}{6} Y^{3}\right)- \\
& -\frac{1}{12 y^{3}}\left(\left(26 x^{3}+62 x^{2}+67 x+62\right) \operatorname{Li}_{2}(-x)-\right. \\
& -\frac{1}{2}\left(151 x^{3}+252 x^{2}+228 x+86\right) X^{2}+ \\
& +\left(57 x^{3}+104 x^{2}+73 x+18\right) X Y- \\
& \left.-\frac{\pi^{2}}{12}\left(44 x^{3}+17 x^{2}-80 x+9\right)\right)-\frac{21 x+8}{8 y} Y^{2}- \\
& -\frac{1}{36 y^{3}}\left(286 x^{3}+314 x^{2}+133 x-30\right) X- \\
& \left.-\frac{1}{36 y^{2}}\left(286 x^{2}+371 x+238\right) Y-\frac{83 x+38}{54 y^{2}}\right] \text {. } \\
& I_{4}^{[3]}=\frac{1}{2 y^{3}}\left[-3(x-y) x \operatorname{Li}_{4}(-y)-\frac{1}{2}\left(8 x^{3}-9 x^{2}-3 x-2\right) \operatorname{Li}_{4}(-x)-\right. \\
& -\left(5 x^{3}+5 x^{2}+4 x+1\right) \operatorname{Li}_{4}\left(-\frac{x}{y}\right)-\left(4 x^{3}+x^{2}+2 x+1\right) X \operatorname{Li}_{3}(-y)- \\
& -\left(x^{3}+5 x^{2}+4 x+2\right) X \operatorname{Li}_{3}(-x)+\left(2 x^{3}+6 x^{2}+3 x+1\right) \zeta_{3} X+ \\
& +(x-y)(1-x y) Y\left(\operatorname{Li}_{3}(-x)-\zeta_{3}\right)+\frac{1}{4}\left(2 x^{3}+x^{2}+5 x+4\right) X^{2} \operatorname{Li}_{2}(-x)- \\
& -\left(x^{3}-x^{2}+x+1\right) X Y \operatorname{Li}_{2}(-x)+ \\
& +\frac{\pi^{2}}{6}\left(x^{3}-x^{2}-2 x-1\right) \operatorname{Li}_{2}(-x)+\frac{1}{24}(3 x-1)(x-y) x X^{4}-
\end{aligned}
$$




$$
\begin{aligned}
& -\frac{1}{24}\left(6 x^{3}+8 x^{2}+7 x+2\right) Y^{4}-\frac{1}{3}\left(x^{3}+3 x^{2}-1\right) X^{3} Y- \\
& -\frac{1}{4}\left(4 x^{3}-5 x^{2}+2 x+3\right) X^{2} Y^{2}+\frac{1}{6}\left(7 x^{3}+12 x^{2}+12 x+4\right) X Y^{3}- \\
& -\frac{\pi^{2}}{12}\left(9 x^{2}+8 x+1\right) x X^{2}+\frac{\pi^{2}}{3}\left(4 x^{2}-2 x-1\right) x X Y- \\
& \left.-\frac{\pi^{2}}{12}\left(4 x^{3}+5 x^{2}+7 x+3\right) Y^{2}+\frac{\pi^{4}}{360}\left(62 x^{2}+140 x+79\right) x\right]+ \\
& +\frac{3 x+1}{2 y} Y \operatorname{Li}_{3}(-y)-\frac{1}{4} Y^{2} \operatorname{Li}_{2}(-x)+ \\
& +\frac{x}{4 y^{3}}\left[3\left(5 x^{2}+4 x+2\right)\left(\operatorname{Li}_{3}(-x)-X \operatorname{Li}_{2}(-x)\right)-\right. \\
& -\frac{2}{3}\left(8 x^{2}+7 x+2\right) X^{3}+\frac{1}{3}\left(6 x^{2}+11 x+1\right) X^{2} Y- \\
& \left.-\frac{\pi^{2}}{6}\left(22 x^{2}+28 x+11\right) X+(2 x-7) \zeta_{3}\right]- \\
& -\frac{x}{4 y^{2}}\left[(9 x+7)\left(\operatorname{Li}_{3}(-y)+Y \operatorname{Li}_{2}(-x)\right)+\right. \\
& \left.+\frac{1}{3}(13 x+10) X Y^{2}-2(x-y) \pi^{2} Y\right]- \\
& -\frac{x}{24 y^{3}}\left(6 x^{3}-3 x^{2}-49 x-37\right) X^{2}-\frac{1}{12 y^{2}}\left(6 x^{3}-15 x^{2}-38 x-24\right) X Y+ \\
& +\frac{\pi^{2}}{8 y^{2}}\left(2 x^{3}-9 x-8\right)-\frac{1}{8 x y}\left(2 x^{3}-9 x^{2}-12 x-2\right) Y^{2}- \\
& -\frac{x}{12 y^{2}}(47 x+41) X-\frac{47 x+46}{12 y} Y+ \\
& +i \pi\left[\frac { 1 } { 2 y ^ { 3 } } \left(\left(3+6 x-x^{2}\right) x \operatorname{Li}_{3}(-y)+\left(x^{3}-2 x^{2}-x-1\right) \operatorname{Li}_{3}(-x)+\right.\right. \\
& +2(x-y) x Y \operatorname{Li}_{2}(-x)+\frac{1}{2}\left(3 x^{2}+3 x+2\right) X \operatorname{Li}_{2}(-x)+ \\
& +\frac{x}{6}(x-1)(3 x+2) X^{3}+\frac{x}{2}\left(x^{2}+10 x+5\right) X Y^{2}- \\
& \left.-\frac{\pi^{2}}{6}\left(3 x^{2}+10 x+5\right) x Y+3 \zeta_{3} x^{2}\right)- \\
& -\frac{1}{4 y}\left(x Y^{3}+(x-1) X^{2} Y+\pi^{2} x X\right)+ \\
& +\frac{x}{4 y^{3}}\left(-\left(6 x^{2}-4 x-1\right) \operatorname{Li}_{2}(-x)-\frac{1}{6}\left(39 x^{2}+26 x+4\right) X^{2}+\right. \\
& \left.+\frac{1}{3}\left(11 x^{2}+20 x+1\right) X Y+\frac{\pi^{2}}{6}\left(2 x^{2}-8 x-1\right)\right)- \\
& -\frac{x}{24 y} Y^{2}-\frac{1}{12 y^{3}}\left(6 x^{3}+4 x^{2}+25 x+24\right) X- \\
& \left.-\frac{1}{12 x y^{2}}\left(6 x^{3}+25 x^{2}+18 x+6\right) Y+\frac{26 x+23}{6 y^{2}}\right] \text {. }
\end{aligned}
$$




$$
\begin{aligned}
& J_{4}^{[3]}=\frac{x(x-1)}{y^{3}}\left[\operatorname{Li}_{4}(-x)+\operatorname{Li}_{4}(-y)-\mathrm{Li}_{4}\left(-\frac{x}{y}\right)-\left(\operatorname{Li}_{3}(-x)-\zeta_{3}\right) X+\right. \\
& +\left(\operatorname{Li}_{3}(-x)-\zeta_{3}\right) Y+\frac{\pi^{2}}{6} \operatorname{Li}_{2}(-x)-\frac{1}{24} X^{4}+\frac{1}{6} X Y^{3}- \\
& \left.-\frac{1}{24} Y^{4}-\frac{\pi^{2}}{6} X^{2}-\frac{\pi^{2}}{12} Y^{2}-\frac{7 \pi^{4}}{120}\right]+ \\
& +\frac{1}{72 y^{3}}\left(x\left(34+11 x-20 x^{2}\right) X^{3}+3(3 x+2)\left(x^{2}-x-3\right) X^{2} Y\right)+ \\
& +\frac{1}{3 y^{2}}\left[\left(x^{2}+9 x+2\right)\left(\operatorname{Li}_{3}(-x)-X \operatorname{Li}_{2}(-x)\right)-\right. \\
& -\left(x^{2}-6 x-1\right)\left(\operatorname{Li}_{3}(-y)+Y \operatorname{Li}_{2}(-x)\right)- \\
& \left.-\frac{1}{8}(x-2)(9 x+1) X Y^{2}+\frac{\pi^{2}}{24}\left(41 x^{2}-22 x-15\right) Y\right]+ \\
& +\frac{\pi^{2}}{36 y}(20 x+33) X+\frac{1}{3 y} \zeta_{3}-\frac{1}{9} Y^{3}+\frac{x}{12 y^{3}}\left(6 x^{3}+44 x^{2}+66 x+25\right) X^{2}+ \\
& +\frac{1}{72 y^{2}}\left[\left(72 x^{3}+303 x^{2}+259 x+22\right) X Y-\left(36 x^{3}+126 x^{2}+139 x+73\right) \pi^{2}\right]+ \\
& +\frac{1}{24 x y}\left(12 x^{3}+13 x^{2}-5 x+6\right) Y^{2}-\frac{x}{72 y^{2}}(19 x-17) X-\frac{19 x-20}{72 y} Y+ \\
& +i \pi\left[\frac{1}{24 y^{3}}\left(2\left(8 x^{3}+11 x^{2}-2 x-4\right) X Y-\left(21 x^{3}+30 x^{2}+21 x+14\right) X^{2}\right)+\right. \\
& +\frac{1}{3 y}\left(\frac{1}{8}(13 x+10) Y^{2}+(x-y) \operatorname{Li}_{2}(-x)-\frac{\pi^{2}}{24}(x-25)\right)+ \\
& +\frac{1}{72 y^{3}}\left(153 x^{3}+230 x^{2}+19 x-22\right) X+ \\
& \left.+\frac{1}{72 x y^{2}}\left(153 x^{3}+211 x^{2}+16 x-36\right) Y+\frac{4 x-5}{18 y^{2}}\right] \text {, } \\
& K_{4}^{[3]}=\frac{x(x-1)}{6 y^{3}}\left[12\left(\mathrm{Li}_{4}(-x)+\mathrm{Li}_{4}(-y)-\mathrm{Li}_{4}\left(-\frac{x}{y}\right)\right)+\right. \\
& +12(Y-X)\left(\operatorname{Li}_{3}(-x)-\zeta_{3}\right)+2 \pi^{2} \operatorname{Li}_{2}(-x)-\frac{1}{2} X^{4}+ \\
& \left.+2 X Y^{3}-\frac{1}{2} Y^{4}-\pi^{2}\left(2 X^{2}+Y^{2}\right)-\frac{7 \pi^{4}}{10}\right]+ \\
& +\frac{1}{3 y^{3}}\left[-\left(x^{3}+15 x^{2}+15 x+2\right)\left(\operatorname{Li}_{3}(-x)-X \operatorname{Li}_{2}(-x)\right)-\right. \\
& -\frac{x}{24}\left(2 x^{2}-30 x-27\right) X^{3}+\frac{1}{8}\left(5 x^{3}+20 x^{2}+22 x+8\right) X^{2} Y- \\
& \left.-\frac{\pi^{2}}{24} x\left(x^{2}-90 x-87\right) X+\zeta_{3}\right]+ \\
& +\frac{1}{3 y^{2}}\left[\left(x^{2}+14 x+1\right) Y\left(\operatorname{Li}_{2}(-x)-\frac{\pi^{2}}{3}\right)+\right. \\
& \left.+\frac{1}{8}\left(7 x^{2}+63 x+8\right) X Y^{2}+\left(x^{2}+14 x+1\right) \operatorname{Li}_{3}(-y)\right]+
\end{aligned}
$$




$$
\begin{aligned}
& +\frac{x}{8 y^{3}}\left(6 x^{3}+23 x^{2}+32 x+13\right) X^{2}+ \\
& +\frac{x}{y^{2}}\left(\frac{1}{8}\left(12 x^{2}+35 x+21\right) X Y-\frac{\pi^{2}}{36}\left(27 x^{2}+81 x+28\right)\right)+ \\
& +\frac{1}{4 y}\left(3 x^{2}+6 x-1\right) Y^{2}-\frac{x(29 x+9)}{24 y^{2}} X-\frac{29 x+8}{24 y} Y+ \\
& +i \pi\left[\frac { 1 } { 3 y ^ { 3 } } \left(\operatorname{Li}_{2}(-x)-\frac{x}{8}\left(x^{2}+10 x+11\right) X^{2}+\frac{1}{4}\left(2 x^{3}+10 x^{2}+11 x+4\right) X Y-\right.\right. \\
& \left.\quad-\frac{\pi^{2}}{24}\left(9 x^{3}+30 x^{2}+33 x+16\right)\right)-\frac{3 x+4}{24 y} Y^{2}- \\
& \left.\quad-\frac{x}{8 y^{3}}\left(x^{2}-8 x-5\right) X-\frac{1}{8 y^{2}}\left(x^{2}-x-4\right) Y+\frac{7 x+2}{6 y^{2}}\right] \\
& \quad \frac{1}{6 y}\left(\frac{1}{12}(4+3 x) X Y-\frac{5}{12} x X^{2}+\frac{5}{9} x X-\frac{\pi^{2}}{2}\right)-\frac{1}{36} Y^{2}+\frac{5}{54} Y+ \\
& +i \frac{\pi}{72 y}\left(-(7 x-4) X+(7 x+8) Y-\frac{20}{3}\right) .
\end{aligned}
$$

For $h=5$ in eq. (2.26) and color factor $\operatorname{Tr}^{[1]}$ in eq. (2.31):

$$
\begin{aligned}
A_{5}^{[1]}=\frac{1}{4 y}[ & -\left(20 x^{3}+17 x-2+36 x^{2}\right) \operatorname{Li}_{4}(-x)+ \\
& +2\left(18 x^{2}+10 x^{3}-1+8 x\right) X \operatorname{Li}_{3}(-x)+\frac{1-y}{2} X^{2} \operatorname{Li}_{2}(-x)- \\
& \quad-\frac{1}{12}\left(10 x^{3}+18 x^{2}+11 x+6\right) X^{4}+ \\
& +\frac{\pi^{2}}{3}\left(5 x^{3}+9 x^{2}+7 x+7\right) X^{2}+(5 x+7) \zeta_{3} X+ \\
& \left.+\frac{\pi^{4}}{720}\left(1280 x^{3}+2304 x^{2}+1105 x+57\right)\right]+ \\
+ & x(4+5 x)\left[\operatorname{Li}_{4}(-y)-\operatorname{Li}_{4}\left(-\frac{x}{y}\right)-X \operatorname{Li}_{3}(-y)-\frac{1}{24} Y^{4}+\right. \\
+ & \left.+\frac{1}{6} Y^{3} X-\frac{1}{4} X^{2} Y^{2}-\frac{\pi^{2}}{12} Y^{2}\right]+ \\
+ & \frac{1}{y}\left[\frac{1}{6}\left(30 x^{2}+28 x-11\right)\left(-\operatorname{Li}_{3}(-x)+X \operatorname{Li}_{2}(-x)+\frac{1}{2} X^{2} Y\right)-\right. \\
& \quad-\frac{1}{36}\left(21 x+30 x^{2}-25\right) X^{3}+\frac{1}{48}\left(240 x^{2}+107 x-205\right) \zeta_{3}+ \\
& \left.+\frac{\pi^{2}}{288}\left(240 x^{2}+159 x-265\right) X\right]+ \\
+ & \frac{1}{288 y^{2}}\left(721 x^{2}+1449 x+368\right) X^{2}-\frac{\pi^{2}}{12}(10 x-3)- \\
- & \frac{1}{96 y}(167+407 x) X+\frac{1}{16} x-\frac{30377}{3456}+
\end{aligned}
$$




$$
\begin{aligned}
& +i \pi\left[\frac { 1 } { 2 y } \left(\left(10 x^{3}+18 x^{2}+8 x-1\right) \operatorname{Li}_{3}(-x)+\frac{1-y}{2} X \operatorname{Li}_{2}(-x)-\right.\right. \\
& -\frac{1}{6}\left(10 x^{3}+18 x^{2}+11 x+6\right) X^{3}- \\
& \left.-\frac{\pi^{2}}{3}\left(5 x^{3}-1+4 x+9 x^{2}\right) X-(x-y) \zeta_{3}\right)- \\
& -x(4+5 x) \operatorname{Li}_{3}(-y)-\frac{1}{4}\left(10 x^{2}+8 x+1\right) X^{2} Y+ \\
& +\frac{1}{6 y}\left(\left(30 x^{2}+28 x-11\right)\left(\operatorname{Li}_{2}(-x)+X Y\right)-\right. \\
& \left.-\frac{1}{2}\left(30 x^{2}+21 x-25\right) X^{2}-\frac{\pi^{2}}{24}\left(120 x^{2}+83 x-73\right)\right)-\frac{9}{4} \zeta_{3}+ \\
& \left.+\frac{1}{144 y^{2}}\left(721 x^{2}+1449 x+368\right) X+\frac{11}{288} \pi^{2}+\frac{5}{2 y}+\frac{407}{96}\right], \\
& B_{5}^{[1]}=\frac{1}{2 y}\left[-\left(7 x^{3}-6 x^{2}-11 x-5\right) \operatorname{Li}_{4}(-x)-x\left(7 x^{2}-6 x-14\right) \operatorname{Li}_{4}(-y)-\right. \\
& -\left(5 x^{3}+12 x^{2}-4\right) \operatorname{Li}_{4}\left(-\frac{x}{y}\right)-2\left(3 x^{2}-3 x-2\right) y Y \operatorname{Li}_{3}(-y)+ \\
& +\left(6 x^{3}+12 x^{2}+x-4\right) Y \operatorname{Li}_{3}(-x)+ \\
& +\left(x^{3}-12 x^{2}-7 x-1\right) X \operatorname{Li}_{3}(-x)+\left(x^{3}+5 x+4\right) X \operatorname{Li}_{3}(-y)+ \\
& +\frac{1}{2}\left(6 x^{2}+3 x+1\right) X^{2} \operatorname{Li}_{2}(-x)-\left(3 x^{2}+3 x+2\right) Y^{2} \operatorname{Li}_{2}(-x)+ \\
& +\frac{\pi^{2}}{6}\left(5 x^{3}+12 x^{2}-18 x-14\right) \operatorname{Li}_{2}(-x)-4 y \operatorname{Li}_{2}(-x) X Y- \\
& -\frac{1}{24}\left(11 x^{3}+8 x+24 x^{2}-2\right) Y^{4}+\frac{1}{24} x\left(2-x^{2}\right) X^{4}- \\
& -\frac{4-x}{4}\left(x^{2}-2 x-2\right) X^{2} Y^{2}+\frac{1}{6}\left(x^{3}+6 x^{2}+5 x+1\right) Y X^{3}+ \\
& +\frac{y^{2}}{6}(11 x-10) Y^{3} X+(4+5 x) Y \zeta_{3}-\frac{1}{2}(9+11 x) X \zeta_{3}+ \\
& +\frac{\pi^{2}}{12}\left(x^{3}-9 x^{2}-14 x+12\right) X^{2}+\frac{\pi^{2}}{12}\left(x^{3}-3 x^{2}+12 x+14\right) Y^{2}- \\
& -\frac{\pi^{2}}{6}\left(2 x^{3}-9 x^{2}+15 x+12\right) Y X+ \\
& \left.+\frac{\pi^{4}}{1440}\left(488 x^{3}-1152 x^{2}-987 x-343\right)\right]+ \\
& +\frac{1}{4 y}\left[x\left(7 x^{2}+16 x+21\right)\left(\operatorname{Li}_{3}(-y)+Y \mathrm{Li}_{2}(-x)\right)+\right. \\
& +x\left(7 x^{2}+2 x+1\right)\left(\operatorname{Li}_{3}(-x)-X \operatorname{Li}_{2}(-x)\right)- \\
& -\frac{x}{18}\left(3 x^{2}+18 x+29\right) X^{3}-\frac{y}{3}\left(6 x^{2}+15 x+10\right) Y^{3}+ \\
& +\frac{2 x}{3}(4+9 x) Y X^{2}+\frac{1}{6}\left(3 x^{3}-12 x^{2}+21 x-40\right) X Y^{2}- \\
& -\frac{1}{36}\left(360 x^{2}+133 x-443\right) \zeta_{3}-\frac{\pi^{2}}{3}\left(x^{3}+x^{2}+26 x-10\right) Y-
\end{aligned}
$$




$$
\begin{aligned}
& \left.-\frac{\pi^{2}}{72}\left(96 x^{3}+72 x^{2}-541 x-477\right) X\right]+ \\
& +\frac{1}{144 y^{2}}\left(99 x^{4}+63 x^{3}-98 x^{2}+154 x+144\right) X^{2}+ \\
& +\frac{x}{72 y}\left(99 x^{2}+73+72 x\right) X Y+\frac{1}{144 x}\left(99 x^{3}+81 x^{2}+173 x+27\right) Y^{2}- \\
& -\frac{\pi^{2}}{288 y}\left(198 x^{3}+108 x^{2}-145 x-455\right)+ \\
& +\frac{1}{216 y}\left(81 x^{2}+647 x+782\right) X-\frac{3}{8}(1-x) Y+\frac{1}{8} x+\frac{19139}{2592}+ \\
& +i \pi\left[\frac { 1 } { 2 y } \left(\left(7 x^{3}-5-6 x\right) \operatorname{Li}_{3}(-x)-2 x(3 x+1) Y \operatorname{Li}_{2}(-x)-\right.\right. \\
& -x\left(5-7 x^{2}\right) \mathrm{Li}_{3}(-y)+\left(6 x^{2}+7 x+5\right) X \mathrm{Li}_{2}(-x)- \\
& -\frac{x}{6}\left(6 x^{2}+6 x+1\right) Y^{3}-\frac{x}{6}\left(x^{2}-6 x-8\right) X^{3}+ \\
& +\left(3 x^{3}-x+1\right) X Y^{2}+\frac{1}{2}\left(x^{3}+2 x+1\right) Y X^{2}- \\
& -\frac{\pi^{2}}{6}\left(6 x^{3}-6 x^{2}-15 x-8\right) Y- \\
& \left.-\frac{\pi^{2}}{6}\left(x^{3}-12 x^{2}-5 x-6\right) X+\frac{1}{2} y \zeta_{3}\right)+ \\
& +\frac{1}{2 y}\left(\operatorname{Li}_{2}(-x) x(7 x+10)+\frac{x}{6}(3 x-1)(3 x+5) X^{2}+\right. \\
& +\frac{1}{6}\left(9 x^{3}+33 x^{2}+54 x+10\right) Y^{2}- \\
& -\frac{1}{3}\left(9 x^{3}+12 x^{2}+13 x+20\right) X Y+ \\
& \left.+\frac{\pi^{2}}{48}\left(72 x^{3}+112 x^{2}+111 x+79\right)\right)- \\
& -\frac{1}{8 y^{2}}\left(27 x^{2}+12 x^{3}-16-9 x\right) X- \\
& \left.-\frac{1}{72 y x}\left(181 x^{2}+108 x^{3}+200 x+27\right) Y+\frac{1}{y}-\frac{647}{216}\right], \\
& C_{5}^{[1]}=\frac{x}{2 y}\left[\frac { 1 } { 2 4 } ( x ^ { 2 } - 2 ) \left(-24 \operatorname{Li}_{4}(-y)+24 \operatorname{Li}_{4}\left(-\frac{x}{y}\right)-4 \pi^{2} \operatorname{Li}_{2}(-x)+\right.\right. \\
& \left.+Y^{4}-4 Y^{3} X+2 \pi^{2} Y^{2}\right)+ \\
& +\frac{1}{2}\left(7-2 x^{2}\right) \operatorname{Li}_{4}(-x)+\left(\operatorname{Li}_{3}(-x)-\zeta_{3}\right) Y- \\
& -y(x-1) X \operatorname{Li}_{3}(-y)+\left(x^{2}-3\right) X \operatorname{Li}_{3}(-x)+\frac{1}{4} \operatorname{Li}_{2}(-x) X^{2}- \\
& \left.-\frac{1}{24}\left(1+x^{2}\right) X^{4}+\frac{\pi^{2}}{12} x^{2} X^{2}\right]+ \\
& +\frac{x}{24}(1-x) X^{2} Y(3 Y+2 X)+\frac{\pi^{2}}{6} x(x-1) Y X-
\end{aligned}
$$




$$
\begin{aligned}
& -\frac{1}{2 y}(x+3) X \zeta_{3}+\frac{\pi^{4}}{720 y}\left(32 x^{3}-48 x+11\right)+ \\
& +\frac{x}{12}(x+3)\left(-3 \operatorname{Li}_{3}(-y)-3 Y \operatorname{Li}_{2}(-x)-\frac{3}{2} X Y^{2}+\pi^{2} Y\right)+ \\
& +\frac{1}{4 y}\left[x\left(7+x^{2}+2 x\right)\left(\operatorname{Li}_{3}(-x)-X \operatorname{Li}_{2}(-x)\right)-\right. \\
& -\frac{x}{6}\left(x^{2}+6 x-1\right) X^{3}+x(x-2) Y X^{2}+ \\
& \left.+\frac{1}{2}\left(4 x^{2}+7 x+15\right) \zeta_{3}-\frac{\pi^{2}}{6}\left(2 x^{3}+6 x^{2}+7 x-3\right) X\right]- \\
& -\frac{x}{32 y^{2}}\left(2 x^{3}+10 x^{2}+21+21 x\right) X^{2}+\frac{x}{8}(x+3) X Y- \\
& -\frac{1}{16 x}\left(x^{3}+3 x^{2}+3 x-3\right) Y^{2}-\frac{\pi^{2}}{96}\left(6 x^{2}+30 x-29\right)+ \\
& +\frac{3}{8}(x-1) Y+\frac{1}{32 y}\left(12 x^{2}+25 x-3\right) X+\frac{x}{16}+\frac{255}{128}+ \\
& +i \pi\left[\frac { 1 } { 2 y } \left(-x\left(2-x^{2}\right) \operatorname{Li}_{3}(-x)+\frac{x}{2} \operatorname{Li}_{2}(-x) X-(2 x+3) \zeta_{3}-\right.\right. \\
& \left.-\frac{x^{3}}{6} X^{3}+\frac{\pi^{2}}{6} x\left(2-x^{2}\right) X\right)+\frac{x}{4}(1-x)\left(2 \operatorname{Li}_{3}(-y)+Y X^{2}\right)+ \\
& +\frac{x(x-2)}{2 y}\left(\operatorname{Li}_{2}(-x)+X Y-\frac{1}{2} X^{2}-\frac{\pi^{2}}{6}\right)-\frac{1}{8} \pi^{2}- \\
& \left.-\frac{x}{16 y^{2}}(7 x+15) X-\frac{3(x-1)}{8 x} Y-\frac{1}{2 y}-\frac{25}{32}\right] \text {, } \\
& D_{5}^{[1]}=x(x-y)\left[-\mathrm{Li}_{4}(-x)-\mathrm{Li}_{4}(-y)+\mathrm{Li}_{4}\left(-\frac{x}{y}\right)+\right. \\
& +\left(\operatorname{Li}_{3}(-x)+\operatorname{Li}_{3}(-y)\right) X-\frac{\pi^{2}}{6} \operatorname{Li}_{2}(-x)-\frac{1}{6} Y^{3} X+ \\
& +\frac{1}{4} X^{2} Y^{2}+\frac{\pi^{2}}{12} Y^{2}+\frac{1}{24} Y^{4}+\frac{\pi^{2}}{12} X^{2}-\frac{1}{24} X^{4}+ \\
& \left.+\frac{1}{6} Y X^{3}-\frac{\pi^{2}}{3} Y X+\frac{4}{45} \pi^{4}\right]- \\
& -\frac{y}{3} \operatorname{Li}_{3}(-x)-\frac{7}{3} x \operatorname{Li}_{3}(-x)-\frac{1}{6}(1-6 x) Y X^{2}- \\
& -\frac{1}{3}(1-6 x) X \operatorname{Li}_{2}(-x)+\frac{1}{72 y}\left(24 x^{2}+15 x-14\right) X^{3}- \\
& -\frac{\pi^{2}}{144 y}\left(48 x^{2}+21 x-47\right) X+\frac{65}{24} \zeta_{3} x+\frac{17}{24} \zeta_{3} y+\frac{\pi^{2}}{27}(4+9 x)- \\
& -\frac{1}{72 y^{2}}\left(70 x^{2}+141 x+35\right) X^{2}+\frac{1}{216 y}(163 x-53) X+\frac{863}{864}+ \\
& +i \pi\left[x(x-y)\left(\left(\operatorname{Li}_{3}(-x)+\operatorname{Li}_{3}(-y)\right)+\frac{1}{2} Y X^{2}-\frac{1}{6} X^{3}-\frac{\pi^{2}}{6} X\right)-\right. \\
& -\frac{1}{3}(1-6 x) \operatorname{Li}_{2}(-x)+\frac{1}{24 y}\left(24 x^{2}+15 x-14\right) X^{2}+
\end{aligned}
$$




$$
\begin{aligned}
& +\frac{1}{3}(6 x-1) X Y-\frac{\pi^{2}}{48}(16 x-3)- \\
& \left.-\frac{1}{36 y^{2}}\left(141 x+70 x^{2}+35\right) X-\frac{1}{y}-\frac{163}{216}\right] \text {, } \\
& E_{5}^{[1]}=x(x-y)\left[2\left(\operatorname{Li}_{4}(-x)+\mathrm{Li}_{4}(-y)-\mathrm{Li}_{4}\left(-\frac{x}{y}\right)\right)-\right. \\
& -2 X\left(\operatorname{Li}_{3}(-x)+\operatorname{Li}_{3}(-y)\right)+\frac{\pi^{2}}{3} \operatorname{Li}_{2}(-x)+\frac{1}{3} Y^{3} X- \\
& -\frac{1}{12} Y^{4}-\frac{1}{3} Y X^{3}-\frac{1}{2} X^{2} Y^{2}+\frac{1}{12} X^{4}-\frac{\pi^{2}}{6} Y^{2}+ \\
& \left.+\frac{2}{3} \pi^{2} Y X-\frac{\pi^{2}}{6} X^{2}-\frac{8}{45} \pi^{4}\right]+ \\
& +\frac{1}{3 y}\left[\left(x^{3}-9 x^{2}-9 x+2\right)\left(\operatorname{Li}_{3}(-x)-X \operatorname{Li}_{2}(-x)\right)+\right. \\
& +\frac{x}{24}\left(8 x^{2}-24 x-19\right) X^{3}+\frac{1}{4}\left(4 x^{3}+15 x^{2}+15 x+6\right) X Y^{2}- \\
& -\frac{1}{8}\left(8 x^{3}-21 x^{2}-23 x+8\right) Y X^{2}-\frac{\pi^{2}}{24}\left(8 x^{3}+33 x^{2}+21 x+14\right) Y+ \\
& \left.+\frac{\pi^{2}}{48}\left(8 x^{3}+120 x^{2}+73 x-27\right) X+\frac{1}{24}\left(288 x^{2}+287 x-25\right) \zeta_{3}\right]- \\
& -\frac{y^{2}}{3}\left(\operatorname{Li}_{3}(-y)+Y \operatorname{Li}_{2}(-x)\right)+\frac{1}{24}(3 x+2) Y^{3}- \\
& -\frac{x}{72 y^{2}}\left(18 x^{3}+24 x^{2}-79 x-139\right) X^{2}-\frac{1}{36}\left(9 x^{2}-18 x+1\right) Y^{2}- \\
& -\frac{1}{144 y}\left[8 x\left(9 x^{2}-3 x-7\right) X Y-\left(36 x^{3}+39 x-33+68 x^{2}\right) \pi^{2}\right]- \\
& -\frac{1}{216 y}\left(191+335 x-180 x^{2}\right) X+\frac{1}{6}(5 x-4) Y-\frac{4085}{2592}+ \\
& +i \pi\left[\frac{x}{3}(x-y)\left(X^{3}-3 Y X^{2}-6 \operatorname{Li}_{3}(-x)-6 \operatorname{Li}_{3}(-y)+\pi^{2} X\right)+\right. \\
& +\frac{1}{3 y}\left(\left(12 x^{2}+12 x-1\right) \operatorname{Li}_{2}(-x)+\frac{x}{8}\left(4 x^{2}-39 x-32\right) X^{2}-\right. \\
& -\frac{1}{8}\left(4 x^{2}+x+1\right)(y-1) Y^{2}-\frac{x}{4}\left(4 x^{2}-39 x-41\right) X Y+ \\
& \left.+\frac{\pi^{2}}{48}\left(24 x^{3}-42 x^{2}-45 x+17\right)\right)-\frac{x}{36 y^{2}}\left(12 x^{2}-59 x-125\right) X- \\
& \left.-\frac{1}{18 y}\left(6 x^{2}+10 x-1\right) Y+\frac{3}{2 y}+\frac{371}{216}\right] \text {, } \\
& F_{5}^{[1]}=F_{4}^{[1]}, \\
& G_{5}^{[1]}=G_{4}^{[1]} \text {. }
\end{aligned}
$$

For $h=5$ in eq. (2.26) and color factor $\operatorname{Tr}^{[2]}$ in eq. (2.31):

$$
A_{5}^{[2]}=\frac{1}{y}\left[\frac { 1 } { 4 8 } ( 1 - y ) ( 1 - x y ) \left(6 X^{2} \operatorname{Li}_{2}(-x)-12 X Y \operatorname{Li}_{2}(-x)+\right.\right.
$$




$$
\begin{aligned}
& \left.+6 Y^{2} \operatorname{Li}_{2}(-x)+3 X^{4}+2 Y^{4}+9 X^{2} Y^{2}+\pi^{2} Y^{2}\right)- \\
& -\left(3 x^{3}+9 x^{2}+9 x+2\right)\left(\frac{1}{4} \operatorname{Li}_{4}\left(-\frac{x}{y}\right)-\frac{\pi^{2}}{24} \operatorname{Li}_{2}(-x)\right)- \\
& -\frac{1}{12}\left(3 x^{3}+9 x^{2}+9 x+5\right) X^{3} Y-\frac{1}{24} X\left(x^{3}+3 x^{2}+3 x+4\right)\left(Y^{3}-\pi^{2} X\right)- \\
& \left.-\frac{\pi^{2}}{6} X Y-\frac{5}{4} \zeta_{3} X-\zeta_{3} Y-\frac{\pi^{4}}{320}\left(4 x^{3}+12 x^{2}+12 x-7\right)\right]+ \\
& +\frac{y^{2}}{2}(X-Y)\left(\operatorname{Li}_{3}(-x)+\operatorname{Li}_{3}(-y)\right)+ \\
& +\frac{1}{24 y}\left(6 x^{2}+15 x+44\right)\left(\operatorname{Li}_{3}(-x)+\operatorname{Li}_{3}(-y)+(Y-X) \operatorname{Li}_{2}(-x)\right)- \\
& -\frac{1}{y}\left[\frac{1}{72}\left(23 x^{3}+60 x^{2}+42 x+50\right) X^{3}-\frac{1}{48}\left(2 x^{3}-18 x^{2}-63 x-32\right) X^{2} Y-\right. \\
& -\frac{1}{24}\left(21 x^{3}+78 x^{2}+105 x+82\right) X Y^{2}+ \\
& +\frac{1}{144}\left(86 x^{3}+282 x^{2}+327 x+208\right) Y^{3}+ \\
& +\frac{\pi^{2}}{288}\left(92 x^{3}+288 x^{2}+288 x+335\right) X+ \\
& \left.+\frac{\pi^{2}}{144}\left(86 x^{3}+276 x^{2}+312 x+267\right) Y+\frac{3}{16}\left(4 x^{2}+10 x-13\right) \zeta_{3}\right]+ \\
& +\frac{1}{288 y}\left[\left(475 x^{3}+798 x^{2}+186 x+368\right) X^{2}-\right. \\
& -2\left(475 x^{3}+1062 x^{2}+846 x+280\right) X Y+ \\
& +\left(475 x^{3}+1326 x^{2}+1506 x+1160\right) Y^{2}+ \\
& \left.+\pi^{2}\left(475 x^{3}+982 x^{2}+622 x-528\right)\right]+ \\
& +\frac{1}{y}\left[\frac{1}{288}\left(950 x^{2}+1433 x+501\right) X-\right. \\
& \left.-\frac{1}{864}\left(2850 x^{2}+5091 x-32\right) Y\right]-\frac{36077}{3456 y}-\frac{475}{288}+ \\
& +i \pi\left[-\frac{9}{4 y} \zeta_{3}-11 \frac{(1-y)}{12 y}(1-x y)\left((X-Y)^{2}+\pi^{2}\right)+\frac{11}{288 y} \pi^{2}+\right. \\
& \left.+\frac{11}{36 y}\left(\left(6 x^{2}+15 x-2\right)(Y-X)+22 Y\right)+\frac{2327}{864 y}+\frac{11}{12}\right] \text {, } \\
& B_{5}^{[2]}=\frac{1}{2 y}\left[-\frac{1}{2}(y-1)\left(4 x^{2}-5 x-5\right) \operatorname{Li}_{4}\left(-\frac{x}{y}\right)-(12 x+13) x \operatorname{Li}_{4}(-y)-\right. \\
& -\left(12 x^{2}+9 x+4\right) \operatorname{Li}_{4}(-x)+\left(x^{3}+9 x^{2}+x-1\right) X \operatorname{Li}_{3}(-x)- \\
& -\left(x^{3}-9 x^{2}-5-14 x\right) Y \operatorname{Li}_{3}(-y)+(x-5) y^{2} X \operatorname{Li}_{3}(-y)- \\
& -\left(x^{3}+3 x^{2}-5-4 x\right) Y \operatorname{Li}_{3}(-x)-\frac{1}{4}\left(9 x^{2}+x-2\right) X^{2} \operatorname{Li}_{2}(-x)-
\end{aligned}
$$




$$
\begin{aligned}
& -\frac{\pi^{2}}{12}\left(4 x^{3}+3 x^{2}-47 x-26\right) \operatorname{Li}_{2}(-x)+\frac{1}{2}(y-1)(3 x+5) X Y \operatorname{Li}_{2}(-x)+ \\
& +\frac{5}{4}\left(3 x^{2}+3 x+2\right) Y^{2} \operatorname{Li}_{2}(-x)+\frac{x}{24}\left(x^{2}-3 x-3\right) X^{4}- \\
& -\frac{1}{6}\left(x^{3}+3 x^{2}+2 x-1\right) X^{3} Y+\frac{1}{8}\left(6 x^{3}-3 x^{2}-29 x-22\right) X^{2} Y^{2}- \\
& -\frac{1}{12}\left(10 x^{3}-21 x^{2}-47 x-28\right) X Y^{3}+ \\
& +\frac{1}{24}\left(5 x^{3}+6 x^{2}-2 x-4\right) Y^{4}-\frac{\pi^{2}}{2}\left(x^{3}+x^{2}-7 x-4\right) X Y+ \\
& +\frac{\pi^{2}}{12}\left(2 x^{3}+6 x^{2}+3 x-12\right) X^{2}+\frac{1}{2}(12 x+11) \zeta_{3} X+ \\
& +\frac{\pi^{2}}{24}\left(4 x^{3}-3 x^{2}-37 x-34\right) Y^{2}-(6 x+5) \zeta_{3} Y+ \\
& \left.+\frac{\pi^{4}}{1440}\left(68 x^{3}+1404 x^{2}+744 x+343\right)\right]+ \\
& +\frac{1}{4 y}\left[-x\left(3 x^{2}+2 x-4\right)\left(\operatorname{Li}_{3}(-x)-X \operatorname{Li}_{2}(-x)\right)-\right. \\
& -x\left(3 x^{2}+2 x+8\right)\left(\operatorname{Li}_{3}(-y)+Y \operatorname{Li}_{2}(-x)\right)- \\
& -\frac{x}{18}\left(41 x^{2}+24 x+15\right) X^{3}+\frac{x}{6}\left(19 x^{2}-6 x-4\right) X^{2} Y+ \\
& +\frac{1}{6}\left(3 x^{3}+36 x^{2}+9 x+40\right) X Y^{2}-\frac{1}{18}\left(43 x^{3}+78 x^{2}+120 x+60\right) Y^{3}- \\
& -\frac{\pi^{2}}{72}\left(128 x^{3}+72 x^{2}+552 x+477\right) X- \\
& \left.-\frac{\pi^{2}}{18}\left(25 x^{3}+54 x^{2}-75 x+60\right) Y+\frac{1}{36}\left(144 x^{2}+36 x-443\right) \zeta_{3}\right]+ \\
& +\frac{1}{72 y}\left[\frac{1}{2}\left(359 x^{3}+186 x^{2}+186 x+144\right) X^{2}-\right. \\
& -\left(359 x^{3}+372 x^{2}+265 x+144\right) X Y+ \\
& \left.+\frac{\pi^{2}}{4}\left(718 x^{3}+784 x^{2}+382 x-167\right)\right]+ \\
& +\frac{1}{144 x y}\left(359 x^{4}+558 x^{3}+344 x^{2}+308 x-27\right) Y^{2}+ \\
& +\frac{1}{216 y}\left(915 x^{2}+402 x-782\right) X- \\
& -\frac{1}{72 y}\left(305 x^{2}+227 x+213\right) Y+\frac{14135}{2592 y}-\frac{139}{72}+ \\
& +i \pi\left[\frac { 1 } { y } \left(\frac{1}{2}\left(6 x^{2}+5 x+4\right) \operatorname{Li}_{3}(-x)+\frac{x}{2}(6 x+5) \mathrm{Li}_{3}(-y)+\right.\right. \\
& +(3 x+1) x Y \operatorname{Li}_{2}(-x)-\left(3 x^{2}+3 x+2\right) X \operatorname{Li}_{2}(-x)+ \\
& +\frac{1}{4} x\left(x^{2}-2\right) X^{2} Y+\frac{x^{3}}{12} Y^{3}+\frac{x y}{12}(x+5) X^{3}- \\
& -\frac{1}{4}\left(x^{3}-6 x^{2}-3 x+2\right) X Y^{2}-\frac{\pi^{2}}{12}\left(x^{3}+12 x^{2}+5 x+6\right) X+
\end{aligned}
$$




$$
\begin{aligned}
& \left.+\frac{\pi^{2}}{12}\left(x^{3}-12 x^{2}-16 x-8\right) Y+\frac{1}{4} \zeta_{3}\right)+ \\
& +\frac{1}{24 y}\left(-72 x \operatorname{Li}_{2}(-x)-x\left(31 x^{2}+36 x+7\right) X^{2}+\right. \\
& +2\left(31 x^{3}+36 x^{2}+29 x+40\right) X Y-\left(31 x^{3}+36 x^{2}+87 x+20\right) Y^{2}- \\
& \left.-\frac{\pi^{2}}{4}\left(124 x^{3}+128 x^{2}+116 x+79\right)\right)-\frac{x}{72 y}(186 x+79) X+ \\
& \left.+\frac{1}{72 x y}\left(186 x^{3}+79 x^{2}+164 x-27\right) Y-\frac{571}{108 y}+\frac{31}{24}\right] \text {, } \\
& C_{5}^{[2]}=\frac{1}{2 y}\left[-\frac{x}{2}\left(5 x^{2}+12 x+2\right) \operatorname{Li}_{4}\left(-\frac{x}{y}\right)-\right. \\
& -x\left(6 x^{2}+6 x+1\right)\left(\operatorname{Li}_{4}(-x)+\operatorname{Li}_{4}(-y)\right)+ \\
& +X\left(2 x^{3} \operatorname{Li}_{3}(-x)+2 x^{3} \operatorname{Li}_{3}(-y)+3 \zeta_{3}\right)+ \\
& +\left(4 x^{2}+6 x+1\right) x Y\left(\operatorname{Li}_{3}(-x)+\operatorname{Li}_{3}(-y)\right)- \\
& -\frac{x^{3}}{4}(X-Y)^{2} \operatorname{Li}_{2}(-x)-\frac{x}{24}\left(9 x^{2}+12 x+2\right) Y^{4}- \\
& -\frac{x^{3}}{24} X^{2}\left(-8 X Y-9 Y^{2}+2 X^{2}\right)+ \\
& +\frac{\pi^{2}}{12}\left(5 x^{2}+12 x+2\right) x \operatorname{Li}_{2}(-x)+\frac{x}{12}\left(15 x^{2}+24 x+4\right) X Y^{3}+ \\
& \left.+\frac{\pi^{2}}{24} x^{3}\left(2 X^{2}-12 X Y+3 Y^{2}\right)+\frac{\pi^{4}}{360}\left(116 x^{3}+90 x^{2}+15 x-11\right)\right]+ \\
& +\frac{1}{8 y}\left[3 x\left(2 x^{2}-2 x+1\right)\left(\operatorname{Li}_{3}(-x)-X \operatorname{Li}_{2}(-x)\right)+\right. \\
& +x\left(2 x^{2}+6 x+5\right)\left(3 \operatorname{Li}_{2}(-x) Y+3 \operatorname{Li}_{3}(-y)-\pi^{2} Y\right)+ \\
& +\frac{x}{3}\left(6 x^{2}-2 x+1\right) X^{3}-\frac{3}{2}\left(4 x^{2}-2 x+1\right) x X^{2} Y+ \\
& +2\left(3 x^{2}+4 x+4\right) x X Y^{2}+\frac{1}{6}(22 x+13) x Y^{3}- \\
& \left.-\frac{y}{3}\left(3 x^{2}+x-3\right) \pi^{2} X-\left(10 x^{2}+19 x+15\right) \zeta_{3}\right]+ \\
& +\frac{1}{16 y}\left[\frac{x}{2}\left(11 x^{2}+22 x-6\right) X^{2}-x\left(11 x^{2}+10 x+4\right) X Y+\right. \\
& \left.+\frac{\pi^{2}}{6}\left(33 x^{3}-26 x^{2}+29\right)\right]+ \\
& +\frac{1}{32 y}\left(46 x^{2}-17 x+3\right) X-\frac{1}{32 y}\left(46 x^{2}-5 x-12\right) Y+ \\
& +\frac{1}{32 x y}\left(11 x^{4}-2 x^{3}-10 x^{2}-8 x-6\right) Y^{2}+\frac{187}{128 y}-\frac{17}{32}+ \\
& +i \pi\left[\frac { 1 } { y } \left(\frac{x}{2}\left(6 x^{2}+6 x+1\right)\left(\operatorname{Li}_{3}(-x)+\operatorname{Li}_{3}(-y)\right)-\right.\right.
\end{aligned}
$$




$$
\begin{aligned}
& \left.-\frac{x^{3}}{12} X^{3}+\frac{x^{3}}{4} X^{2} Y-\frac{\pi^{2}}{12} x^{3} X+\frac{3}{2} \zeta_{3}\right)- \\
& -\frac{x}{12}(5 x+1) Y\left(3 X Y-Y^{2}-\pi^{2}\right)+ \\
& +\frac{1}{y}\left(\frac{3}{2}(x-y) x \operatorname{Li}_{2}(-x)+\frac{\pi^{2}}{24}\left(9 x^{3}-2 x^{2}+x-3\right)+\right. \\
& \left.+\frac{x}{8}\left(3 x^{2}-2 x+1\right) X(X-2 Y)\right)-\frac{x}{8}(3 x+7) Y^{2}+ \\
& \left.+\frac{x}{8 y}(6 x-5) X+\frac{1}{8 x}\left(6 x^{2}+x+3\right) Y+\frac{27}{32 y}+\frac{3}{8}\right] \text {, } \\
& D_{5}^{[2]}=\frac{1}{3 y}\left[-\mathrm{Li}_{3}(-x)-\mathrm{Li}_{3}(-y)+X \operatorname{Li}_{2}(-x)-Y \operatorname{Li}_{2}(-x)+\right. \\
& +\frac{1}{24}\left(5 x^{3}+15 x^{2}+15 x+14\right) X^{3}-\frac{1}{8}(y-1)(x y-1) X^{2} Y+ \\
& +\frac{7}{24}(y-1)(x y-1) Y^{3}-\frac{1}{8}\left(3 x^{3}+9 x^{2}+9 x+10\right) X Y^{2}+ \\
& +\frac{\pi^{2}}{48}\left(10 x^{3}+30 x^{2}+30 x+37\right) X+ \\
& \left.+\frac{7}{24}\left(x^{3}+3 x^{2}+3 x+3\right) \pi^{2} Y-\frac{9}{8} \zeta_{3}\right]+ \\
& +\frac{1}{72 y}\left[-\left(37 x^{3}+60 x^{2}+15 x+35\right) X^{2}+2\left(37 x^{3}+72 x^{2}+45 x+9\right) X Y-\right. \\
& \left.-\left(37 x^{3}+84 x^{2}+75 x+71\right) Y^{2}-\frac{\pi^{2}}{3}\left(111 x^{3}+210 x^{2}+120 x-191\right)\right]- \\
& -\frac{1}{216 y}\left(222 x^{2}+294 x-53\right) X+\frac{1}{36 y}\left(37 x^{2}+55 x+28\right) Y+\frac{1307}{864 y}+\frac{37}{72}+ \\
& +i \pi\left[\frac{1-y}{6 y}(1-x y)\left((X-Y)^{2}+\pi^{2}\right)-\frac{1}{144 y} \pi^{2}+\right. \\
& \left.+\frac{1}{18 y}\left(\left(6 x^{2}+15 x-13\right) X-\left(6 x^{2}+15 x+31\right) Y\right)+\frac{185}{216 y}-\frac{1}{6}\right], \\
& E_{5}^{[2]}=x(x-y)\left[2 \operatorname{Li}_{4}(-x)+2 \operatorname{Li}_{4}(-y)-2 \operatorname{Li}_{4}\left(-\frac{x}{y}\right)-\right. \\
& -2 X\left(\operatorname{Li}_{3}(-x)+\operatorname{Li}_{3}(-y)\right)+\frac{\pi^{2}}{3} \operatorname{Li}_{2}(-x)+ \\
& +\frac{1}{12} X^{4}-\frac{1}{3} X^{3} Y-\frac{1}{2} X^{2} Y^{2}+\frac{1}{3} X Y^{3}- \\
& \left.-\frac{1}{12} Y^{4}-\frac{\pi^{2}}{6}\left(X^{2}-4 X Y+Y^{2}\right)-\frac{8}{45} \pi^{4}\right]+ \\
& +\frac{1}{3 y}\left[\left(x^{3}-9 x^{2}-9 x+2\right)\left(\operatorname{Li}_{3}(-x)-X \operatorname{Li}_{2}(-x)\right)+\right. \\
& +\frac{1}{24}\left(7 x^{3}+9 x^{2}+15 x+6\right) Y^{3}+\frac{1}{24} x\left(13 x^{2}-24 x-24\right) X^{3}- \\
& -\frac{1}{8}\left(9 x^{3}-27 x^{2}-25 x+8\right) X^{2} Y-\frac{1}{8}(y-1)\left(5 x^{2}+8 x+2\right) X Y^{2}+
\end{aligned}
$$




$$
\begin{aligned}
& +\frac{\pi^{2}}{16}\left(6 x^{3}+40 x^{2}+48 x+9\right) X+ \\
& \left.+\frac{\pi^{2}}{24}(y-1)\left(x^{2}+13 x+1\right) Y+\frac{1}{24}\left(288 x^{2}+288 x-23\right) \zeta_{3}\right]- \\
& -\frac{y^{2}}{3}\left(\operatorname{Li}_{3}(-y)+Y \operatorname{Li}_{2}(-x)\right)+\frac{x}{72 y^{2}}\left(x^{3}-11 x^{2}+57 x+123\right) X^{2}+ \\
& +\frac{1}{144 y}\left[4\left(x^{2}+12 x+31\right) x X Y-2\left(x^{3}+36 x^{2}+65 x+38\right) Y^{2}-\right. \\
& \left.-\left(2 x^{3}-60 x^{2}-36 x-33\right) \pi^{2}\right]-\frac{1}{36 y}\left(11 x^{2}+8 x-36\right) Y+ \\
& +\frac{1}{216 y}\left(66 x^{2}-96 x+191\right) X-\frac{3401}{2592 y}+\frac{19}{72}+ \\
& +i \pi\left[x(x-y)\left(-2\left(\operatorname{Li}_{3}(-x)+\operatorname{Li}_{3}(-y)\right)+\frac{1}{3} X^{3}-X^{2} Y+\frac{\pi^{2}}{3} X\right)+\right. \\
& +\frac{1}{y}\left(\frac{1}{3}\left(12 x^{2}+12 x-1\right) \operatorname{Li}_{2}(-x)+\frac{x}{24}(x-5)(8 x+7) X^{2}-\right. \\
& -\frac{1}{12}\left(8 x^{3}-33 x^{2}-31 x+8\right) X Y+ \\
& \left.+\frac{1}{24}\left(8 x^{3}+15 x^{2}+21 x+6\right) Y^{2}+\frac{\pi^{2}}{144}\left(48 x^{3}-6 x^{2}+47-6 x\right)\right)- \\
& -\frac{x}{18 y^{2}}\left(12 x^{2}-7 x-46\right) X-\frac{1}{18 y}\left(12 x^{2}+17 x+19\right) Y+ \\
& \left.+\frac{551}{216 y}+\frac{2}{3}\right] \\
& F_{5}^{[2]}=F_{4}^{[2]}, \\
& G_{5}^{[2]}=G_{4}^{[2]} \text {. }
\end{aligned}
$$

For $h=5$ in eq. (2.26) and color factor $\operatorname{Tr}^{[3]}$ in eq. (2.31):

$$
\begin{aligned}
H_{5}^{[3]}=\frac{1}{2 y}[ & -\left(33 x^{3}+66 x^{2}+41 x-5\right)\left(\operatorname{Li}_{4}(-x)+\operatorname{Li}_{4}(-y)\right)- \\
& -21 \operatorname{Li}_{4}(-y)+\frac{1}{2}\left(44 x^{3}+63 x^{2}-7 x-10\right) \operatorname{Li}_{4}\left(-\frac{x}{y}\right)+ \\
& +\left(28 x^{3}+51 x^{2}+22 x-2\right) X \operatorname{Li}_{3}(-x)+ \\
& +\left(24 x^{3}+39 x^{2}+5 x-1\right) X \operatorname{Li}_{3}(-y)+ \\
& +\left(3 x^{3}+9 x^{2}+14 x+1\right) Y \operatorname{Li}_{3}(-x)+ \\
& +\left(7 x^{3}+21 x^{2}+29 x+13\right) Y \operatorname{Li}_{3}(-y)- \\
& -\frac{1}{4}(x+3)\left(2 x^{2}+3 x+4\right) X{ }^{2} \operatorname{Li}_{2}(-x)- \\
& -\frac{1}{2}\left(2 x^{3}+3 x^{2}+11 x-6\right) X Y \operatorname{Li}_{2}(-x)+ \\
& +\frac{1}{4}\left(6 x^{3}+15 x^{2}+27 x+10\right) Y^{2} \operatorname{Li}_{2}(-x)-
\end{aligned}
$$




$$
\begin{aligned}
& -\frac{\pi^{2}}{12}\left(44 x^{3}+63 x^{2}-31 x-22\right) \operatorname{Li}_{2}(-x)-\frac{x}{24}\left(26 x^{2}+33 x+11\right) X^{4}+ \\
& +\frac{1}{6}\left(24 x^{3}+27 x^{2}-6 x-22\right) X^{3} Y+\frac{1}{8}\left(50 x^{3}+105 x^{2}+29 x+26\right) X^{2} Y^{2}- \\
& -\frac{1}{12}\left(30 x^{3}+39 x^{2}-59 x-36\right) X Y^{3}+\frac{1}{12}\left(11 x^{3}+18 x^{2}+2 x-1\right) Y^{4}+ \\
& +\frac{\pi^{2}}{6}\left(14 x^{3}+27 x^{2}+26 x+18\right) X^{2}-\frac{\pi^{2}}{6}\left(52 x^{3}+93 x^{2}+33 x+10\right) X Y- \\
& -\frac{\pi^{2}}{24} y\left(50 x^{2}+43 x-38\right) Y^{2}+(9 x+1) \zeta_{3} X-(9 x+2) \zeta_{3} Y+ \\
& \left.+\frac{\pi^{4}}{360}\left(985 x^{3}+1875 x^{2}+900 x+184\right)\right]+ \\
& +\frac{1}{12 y}\left[\left(31 x^{3}-60 x^{2}-20 x+124\right)\left(\operatorname{Li}_{3}(-x)-X \operatorname{Li}_{2}(-x)\right)+\right. \\
& +\left(31 x^{3}+114 x^{2}+119 x+62\right)\left(\operatorname{Li}_{3}(-y)+Y \operatorname{Li}_{2}(-x)\right)+ \\
& +\frac{x}{3}\left(40 x^{2}-63 x-35\right) X^{3}-\frac{y}{3}\left(3 x^{2}-30 x-22\right) Y^{3}- \\
& -\left(36 x^{3}-24 x^{2}-14 x+19\right) X^{2} Y+ \\
& +\left(32 x^{3}+102 x^{2}+101 x+41\right) X Y^{2}+ \\
& +\frac{\pi^{2}}{12}\left(98 x^{3}+192 x^{2}-275 x-780\right) X- \\
& \left.-\frac{\pi^{2}}{12}\left(112 x^{3}+588 x^{2}+257 x+307\right) Y+\left(132 x^{2}+123 x-62\right) \zeta_{3}\right]+ \\
& +\frac{x}{72 y^{2}}\left(135 x^{3}+24 x^{2}+268 x+487\right) X^{2}+ \\
& +\frac{1}{72 y}\left[6\left(45 x^{3}+14 x^{2}+x+10\right) X Y-\left(135 x^{3}-34 x^{2}-178 x+250\right) \pi^{2}\right]+ \\
& +\frac{1}{72}\left(135 x^{2}+60 x-172\right) Y^{2}+\frac{x}{108 y}(90 x-121) X+\frac{1}{54}(45 x-38) Y+ \\
& +i \pi\left[\frac { 1 } { 2 y } \left(\left(31 x^{3}+60 x^{2}+34 x+12\right) \operatorname{Li}_{3}(-y)+\right.\right. \\
& +\left(31 x^{3}+60 x^{2}+36 x-1\right) \operatorname{Li}_{3}(-x)- \\
& -\left(2 x^{3}+6 x^{2}+12 x+3\right) X \operatorname{Li}_{2}(-x)+ \\
& +2(1-y)(2-x y) Y \operatorname{Li}_{2}(-x)- \\
& -\frac{1}{3}\left(14 x^{3}+24 x^{2}+13 x+4\right) X^{3}+ \\
& +\frac{1}{2}\left(26 x^{3}+42 x^{2}+9 x-11\right) X^{2} Y+ \\
& +\frac{1}{2}\left(5 x^{3}+18 x^{2}+22 x+22\right) X Y^{2}- \\
& -\frac{1}{6}\left(3 x^{3}+12 x^{2}+9 x+2\right) Y^{3}-(5 x-1) y^{2} \pi^{2} X- \\
& \left.-\frac{1}{6}(1-y)\left(7 x^{2}+10 x+13\right) \pi^{2} Y-\zeta_{3}\right)+
\end{aligned}
$$




$$
\begin{aligned}
& +\frac{1}{y}\left(\frac{1}{12}\left(174 x^{2}+139 x-62\right) \operatorname{Li}_{2}(-x)+\right. \\
& +\frac{1}{24}\left(39 x^{3}-138 x^{2}-62 x+86\right) X^{2}- \\
& -\frac{1}{4}\left(13 x^{3}-46 x^{2}-37 x+6\right) X Y+ \\
& +\frac{1}{8}\left(13 x^{3}+12 x^{2}-7 x-8\right) Y^{2}+ \\
& \left.+\frac{\pi^{2}}{144}\left(234 x^{3}-180 x^{2}-136 x+9\right)\right)- \\
& -\frac{1}{36 y^{2}}\left(153 x^{3}-223 x^{2}-454 x+30\right) X- \\
& \left.-\frac{1}{36 y} Y\left(153 x^{2}-115 x-202\right)+\frac{211}{108 y}+\frac{5}{4}\right], \\
& I_{5}^{[3]}=\frac{1}{y}\left[-\frac{3}{2}\left(x^{2}-1\right) x \operatorname{Li}_{4}(-y)-\frac{1}{2}\left(3 x^{3}+x-1\right) \operatorname{Li}_{4}(-x)-\right. \\
& -\frac{1}{4}\left(16 x^{3}+9 x^{2}+3 x+2\right) \operatorname{Li}_{4}\left(-\frac{x}{y}\right)-\frac{1}{2}\left(2 x^{3}+3 x^{2}+2 x+2\right) X \operatorname{Li}_{3}(-x)- \\
& -\frac{1}{2}\left(6 x^{3}+3 x^{2}+x+1\right) X \operatorname{Li}_{3}(-y)+\frac{1}{2}\left(3 x^{3}+3 x^{2}+2 x+1\right) Y \operatorname{Li}_{3}(-x)+ \\
& +\frac{1}{2}\left(7 x^{3}+3 x^{2}-x+1\right) Y \operatorname{Li}_{3}(-y)-\frac{1}{8}\left(2 x^{3}-3 x^{2}-7 x-4\right) X^{2} \operatorname{Li}_{2}(-x)+ \\
& +\frac{\pi^{2}}{24}\left(16 x^{3}+9 x^{2}-5 x-2\right) \operatorname{Li}_{2}(-x)+ \\
& +\frac{1}{48}(x-1)(4 x+1) x X^{4}-\frac{1}{12}(x-y)\left(3 x^{2}-3 x-2\right) X^{3} Y- \\
& -\frac{1}{16}\left(10 x^{3}+15 x^{2}+13 x+6\right) X^{2} Y^{2}+\frac{1}{24}\left(30 x^{3}+21 x^{2}+5 x+8\right) X Y^{3}- \\
& -\frac{1}{24}\left(4 x^{3}+3 x^{2}+2 x+1\right) Y^{4}-\frac{\pi^{2}}{24}\left(2 x^{2}+3 x+10\right) x X^{2}+ \\
& +\frac{\pi^{2}}{6}\left(4 x^{2}+3 x+3\right) x X Y-\frac{\pi^{2}}{48}\left(10 x^{3}+9 x^{2}+7 x+6\right) Y^{2}+ \\
& \left.+\frac{1}{2}(x-y) \zeta_{3} X-\frac{1}{2}(2 x+1) \zeta_{3} Y+\frac{\pi^{4}}{720}\left(25 x^{2}-27 x+10\right) x\right]- \\
& -\frac{1}{8}\left(6 x^{2}-3 x+2\right) Y^{2} \operatorname{Li}_{2}(-x)+\frac{1}{4}\left(2 x^{2}+x+2\right) X Y \operatorname{Li}_{2}(-x)+ \\
& +\frac{1}{y}\left[-\frac{x}{4}\left(9 x^{2}+2 x-1\right)\left(\operatorname{Li}_{3}(-y)+\operatorname{Li}_{2}(-x) Y\right)-\right. \\
& -\frac{3}{4}\left(3 x^{2}+2\right) x\left(\operatorname{Li}_{3}(-x)-X \operatorname{Li}_{2}(-x)\right)- \\
& -\frac{x}{6}\left(3 x^{2}-3 x+2\right) X^{3}-\frac{1}{12}(26 x+3) x^{2} X Y^{2}+ \\
& +\frac{1}{12}\left(22 x^{2}-9 x+11\right) x X^{2} Y-\frac{\pi^{2}}{24}\left(3 x^{2}-6 x-5\right) x X+ \\
& \left.+\frac{\pi^{2}}{24}\left(20 x^{2}+4 x-1\right) x Y-\frac{x}{4}(16 x+1) \zeta_{3}\right]+
\end{aligned}
$$




$$
\begin{aligned}
& +\frac{x y}{12} Y^{3}+\frac{x}{24 y^{2}}\left(3 x^{3}-16 x^{2}-62 x-37\right) X^{2}+ \\
& +\frac{1}{12 y}\left(3 x^{3}-26 x^{2}-47 x-24\right) X Y- \\
& -\frac{\pi^{2}}{8 y}\left(x^{3}-10 x^{2}-12 x-8\right)-\frac{x}{12 y}(6 x-41) X+ \\
& +\frac{1}{8 x}\left(x^{3}-12 x^{2}-6 x-2\right) Y^{2}-\frac{1}{6}(3 x-23) Y+ \\
& +i \pi\left[\frac { 1 } { 2 y } \left((x-1)\left(x^{2}+x+1\right) \operatorname{Li}_{3}(-x)+\left(x^{2}-2\right) x \operatorname{Li}_{3}(-y)-\right.\right. \\
& -\left(2 x^{3}-2 x-1\right) X \operatorname{Li}_{2}(-x)-2\left(1-x^{2}\right) x \operatorname{Li}_{2}(-x) Y- \\
& -\frac{1}{2} x^{3} Y^{3}+\frac{x}{6}\left(2 x^{2}+1\right) X^{3}+\frac{x}{2}\left(5 x^{2}-4\right) X Y^{2}- \\
& \left.-\frac{x-1}{2}(x-y)^{2} X^{2} Y-\frac{\pi^{2}}{2} x X-\frac{\pi^{2}}{6} x\left(7 x^{2}-4\right) Y\right)+ \\
& +\frac{1}{4 y}\left(-(2 x-7) x \operatorname{Li}_{2}(-x)-\frac{x}{6}\left(19 x^{2}-18 x+20\right) X^{2}-\right. \\
& -\frac{x}{6}\left(19 x^{2}-12 x-3\right) Y^{2}-\frac{\pi^{2}}{6}\left(19 x^{2}-6 x+6\right) x+ \\
& \left.+\frac{x}{3}\left(19 x^{2}-18 x+19\right) X Y\right)+ \\
& +\frac{1}{12 y^{2}}\left(7 x^{3}+11 x^{2}+34 x+24\right) X+ \\
& \left.+\frac{1}{12 x y}\left(7 x^{3}+7 x^{2}+6\right) Y-\frac{47}{12 y}-\frac{1}{12}\right] \text {, } \\
& J_{5}^{[3]}=x(x-y)\left[-\operatorname{Li}_{4}(-x)-\operatorname{Li}_{4}(-y)+\operatorname{Li}_{4}\left(-\frac{x}{y}\right)+X\left(\operatorname{Li}_{3}(-x)+\operatorname{Li}_{3}(-y)\right)-\right. \\
& -\frac{\pi^{2}}{6} \operatorname{Li}_{2}(-x)-\frac{1}{24} X^{4}+\frac{1}{6} X^{3} Y+\frac{1}{4} X^{2} Y^{2}-\frac{1}{6} X Y^{3}+\frac{1}{24} Y^{4}+ \\
& \left.+\frac{\pi^{2}}{12} X^{2}-\frac{\pi^{2}}{3} X Y+\frac{\pi^{2}}{12} Y^{2}+\frac{4}{45} \pi^{4}\right]+ \\
& +\frac{1}{3 y}\left[\left(6 x^{2}+5 x-2\right)\left(\operatorname{Li}_{3}(-x)-X \operatorname{Li}_{2}(-x)\right)+\right. \\
& +(x-1)\left(\operatorname{Li}_{3}(-y)+Y \operatorname{Li}_{2}(-x)\right)- \\
& -\left(6 x^{2}+6 x-1\right) \zeta_{3}+\frac{x}{24}\left(3 x^{2}+57 x+34\right) X^{3}- \\
& -\frac{y}{6}(3 x+2) Y^{3}-\frac{1}{8}\left(2 x^{3}+42 x^{2}+37 x+6\right) X^{2} Y+ \\
& +\frac{1}{8}\left(x^{3}+3 x^{2}+11 x-2\right) X Y^{2}+\frac{\pi^{2}}{24}(4 x-3)(3 x-5) Y+ \\
& \left.+\frac{\pi^{2}}{24}\left(3 x^{3}+9 x^{2}+46 x+66\right) X\right]+ \\
& +\frac{x}{12 y^{2}}\left(3 x^{3}+13 x^{2}-9 x-25\right) X^{2}+
\end{aligned}
$$




$$
\begin{aligned}
& +\frac{1}{72 y}\left[\left(36 x^{3}+126 x^{2}+39 x-22\right) X Y-\left(18 x^{3}+90 x^{2}+72 x-73\right) \pi^{2}\right]+ \\
& +\frac{1}{24 x}\left(6 x^{3}+16 x^{2}+23 x-6\right) Y^{2}-\frac{x}{72 y}(36 x+17) X-\frac{1}{18}(9 x+5) Y+ \\
& +i \pi\left[x(x-y)\left(\left(\operatorname{Li}_{3}(-x)+\operatorname{Li}_{3}(-y)\right)-\frac{1}{6} X^{3}+\frac{1}{2} X^{2} Y-\frac{\pi^{2}}{6} X\right)-\right. \\
& -\frac{1}{3 y}\left(\left(6 x^{2}+4 x-1\right) \operatorname{Li}_{2}(-x)-\frac{1}{8}\left(x^{3}+39 x^{2}+17 x-14\right) X^{2}+\right. \\
& +\frac{1}{4}\left(x^{3}+39 x^{2}+30 x+4\right) X Y-\frac{1}{8}\left(x^{3}+15 x^{2}+27 x+10\right) Y^{2}- \\
& \left.-\frac{\pi^{2}}{24}\left(3 x^{3}+69 x^{2}+65 x+25\right)\right)- \\
& -\frac{1}{72 y^{2}}\left(6 x^{3}+273 x^{2}+317 x-22\right) X- \\
& \left.-\frac{1}{72 x y}\left(6 x^{3}+195 x^{2}+124 x-36\right) Y-\frac{19}{72 y}-\frac{13}{24}\right], \\
& K_{5}^{[3]}=x(x-y)\left[2\left(-\mathrm{Li}_{4}(-x)-\mathrm{Li}_{4}(-y)+\mathrm{Li}_{4}\left(-\frac{x}{y}\right)\right)-\frac{\pi^{2}}{3} \mathrm{Li}_{2}(-x)+\right. \\
& +2\left(\operatorname{Li}_{3}(-x)+\operatorname{Li}_{3}(-y)\right) X-\frac{1}{12}\left(X^{4}-Y^{4}\right)+ \\
& \left.+\frac{1}{3} X Y\left(X^{2}-Y^{2}\right)+\frac{1}{2} X^{2} Y^{2}+\frac{\pi^{2}}{6}(X-Y)^{2}-\frac{\pi^{2}}{3} X Y+\frac{8}{45} \pi^{4}\right]+ \\
& +\frac{1}{3 y}\left[-\left(x^{3}-9 x^{2}-9 x+2\right)\left(\operatorname{Li}_{3}(-x)-X \operatorname{Li}_{2}(-x)\right)-\right. \\
& -\frac{x}{24}\left(5 x^{2}-24 x-27\right) X^{3}+\frac{1}{8}\left(6 x^{3}-24 x^{2}-25 x+8\right) X^{2} Y+ \\
& +\frac{1}{8}(y-1)\left(7 x^{2}+10 x+4\right) X Y^{2}- \\
& \left.-\frac{\pi^{2}}{24} x\left(x^{2}+60 x+60\right) X-\left(12 x^{2}+12 x-1\right) \zeta_{3}\right]+ \\
& +\frac{y^{2}}{3}\left(\operatorname{Li}_{3}(-y)+\operatorname{Li}_{2}(-x) Y-\frac{\pi^{2}}{3} Y\right)+\frac{1}{4}\left(x^{2}-2 x-1\right) Y^{2}+ \\
& +\frac{x}{8 y^{2}}\left(2 x^{3}+2 x^{2}-7 x-13\right) X^{2}+\frac{x}{8 y}\left(4 x^{2}-2 x-5\right) X Y+ \\
& +\frac{\pi^{2}}{36} x(9 x+8)-\frac{x}{24 y}(20 x-9) X-\frac{1}{6}(5 x-2) Y+ \\
& +i \pi\left[x(x-y)\left(2\left(\operatorname{Li}_{3}(-x)+\operatorname{Li}_{3}(-y)\right)-\frac{1}{3} X^{3}+X^{2} Y-\frac{\pi^{2}}{3} X\right)+\right. \\
& +\frac{1}{y}\left(-\frac{1}{3}\left(12 x^{2}+12 x-1\right) \operatorname{Li}_{2}(-x)+\right. \\
& +\frac{1}{12}\left(3 x^{3}-36 x^{2}-37 x+4\right) X Y-\frac{1}{24}\left(3 x^{3}+12 x^{2}+12 x+4\right) Y^{2}- \\
& \left.-\frac{x}{24}\left(3 x^{2}-36 x-38\right) X^{2}-\frac{\pi^{2}}{72}\left(3 x^{2}-4\right)(3 x-4)\right)+
\end{aligned}
$$




$$
\left.+\frac{x}{8 y^{2}}\left(2 x^{2}-7 x-21\right) X+\frac{1}{8 y}\left(2 x^{2}+7 x+4\right) Y-\frac{29}{24 y}-\frac{7}{8}\right],
$$

$L_{5}^{[3]}=L_{4}^{[3]}$.

\section{B. Auxiliary functions for two-loop scheme shifts}

In this appendix we present auxiliary functions appearing in eqs. (4.3) and (4.4) for the shift in the two-loop amplitudes under scheme changes. These functions correspond to the $\delta_{R}$-dependent parts of the $\mathcal{O}(\epsilon)$ terms in the one-loop amplitude remainders. They are given by,

$$
\begin{aligned}
M_{1}^{(1),[1] \epsilon, \delta_{R}}= & N\left(\frac{1}{4}+\frac{x}{6}\right)+\frac{1}{4 N} \\
M_{1}^{(1),[2] \epsilon, \delta_{R}}= & N \frac{x(2 x-1)}{12 y}-\frac{x}{4 y N} \\
M_{1}^{(1),[3] \epsilon, \delta_{R}}= & 0 \\
M_{2}^{(1),[1] \epsilon, \delta_{R}}= & \frac{1}{4 y^{3}}\left[N\left(x X^{2}+y(1-x) X+y^{2}(2+3 x)+i \pi x(2 X-y(3+x))\right)+\right. \\
& +\frac{1}{N}\left(-x^{2} X^{2}-y(1+3 x) X-y^{2}(2+x)-\right. \\
M_{2}^{(1),[2] \epsilon, \delta_{R}}= & \frac{x}{4 y}\left(-N(3+i \pi)+\frac{1}{N}(1+i \pi)\right) \\
M_{2}^{(1),[3] \epsilon, \delta_{R}}= & -\frac{x}{4 y^{2}}\left(X^{2}+2 i \pi X\right) \\
M_{3}^{(1),[1] \epsilon, \delta_{R}}= & N\left(\frac{1}{4}+\frac{1}{6 x}\right)+\frac{1}{4 N} \\
M_{3}^{(1),[2] \epsilon, \delta_{R}}= & N \frac{(2-x)}{12 x y}-\frac{1}{4 y N}, \\
M_{3}^{(1),[3] \epsilon, \delta_{R}}= & 0 \\
M_{4}^{(1),[1] \epsilon, \delta_{R}}= & \frac{1}{4 y^{3}}\left[N\left(x^{2} X^{2}+y(1+3 x) X+y^{2}(3+2 x)+i \pi\left(2 x^{2} X+x y(1-x)\right)\right)\right. \\
M_{5}^{(1),[1],[2] \epsilon \epsilon, \delta_{R}}= & \frac{N}{4}\left[x^{2}\left((X-Y)^{2}+\pi^{2}\right)+X(2 x-1)+\frac{x}{y}(2 x+1) Y-\frac{x+3}{4}=\right. \\
M_{4}^{(1),[3] \epsilon, \delta_{R}}= & \frac{1}{4 y}\left(N(X-3)-\frac{1}{N}(X-1)\right) \\
M^{2}\left(X^{2}+2 i \pi X\right) & \frac{1}{4} N(X-3)-\frac{1}{4 N}(X-1) \\
= &
\end{aligned}
$$




$$
\begin{gathered}
+\frac{1}{4 N}\left[x(1+x) X^{2}-2 x(1+x) X Y+\pi^{2} x(1+x)+X(2 x+1)+\right. \\
\left.+x(1+x) Y^{2}+\frac{x}{y}(2 x+3) Y+\frac{1-x}{y}\right], \\
M_{5}^{(1),[3] \epsilon, \delta_{R}}=-\frac{x}{4}\left((X-Y)^{2}+\pi^{2}\right) .
\end{gathered}
$$

\section{References}

[1] Z. Bern, J.S. Rozowsky and B. Yan, "Two-loop four-gluon amplitudes in $N=4$ super-Yang-Mills," Phys. Lett. B 401, 273 (1997) [arXiv:hep-ph/9702424];

Z. Bern, L.J. Dixon, D.C. Dunbar, M. Perelstein and J.S. Rozowsky, "On the relationship between Yang-Mills theory and gravity and its implication for ultraviolet divergences," Nucl. Phys. B 530, 401 (1998) [arXiv:hep-th/9802162].

[2] Z. Bern, L.J. Dixon and D.A. Kosower, "A two-loop four-gluon helicity amplitude in QCD," JHEP 0001, 027 (2000) [arXiv:hep-ph/0001001].

[3] Z. Bern, L.J. Dixon and A. Ghinculov, "Two-loop correction to Bhabha scattering," Phys. Rev. D 63, 053007 (2001) [arXiv:hep-ph/0010075].

[4] C. Anastasiou, E.W.N. Glover, C. Oleari and M.E. Tejeda-Yeomans, "Two-loop QCD corrections to $q \bar{q} \rightarrow q^{\prime} \bar{q}^{\prime}$," Nucl. Phys. B 601, 318 (2001) [hep-ph/0010212];

"Two-loop QCD corrections to $q \bar{q} \rightarrow q \bar{q}, "$ Nucl. Phys. B 601, 341 (2001) [hep-ph/0011094].

[5] C. Anastasiou, E.W.N. Glover, C. Oleari and M.E. Tejeda-Yeomans, "Two-loop QCD corrections to massless quark-gluon scattering," Nucl. Phys. B 605, 486 (2001) [hep-ph/0101304].

[6] E.W.N. Glover, C. Oleari and M.E. Tejeda-Yeomans, "Two-loop QCD corrections to gluon-gluon scattering," Nucl. Phys. B 605, 467 (2001) [arXiv:hep-ph/0102201].

[7] Z. Bern, A. De Freitas and L.J. Dixon, "Two-loop amplitudes for gluon fusion into two photons," JHEP 0109, 037 (2001) [arXiv:hep-ph/0109078].

[8] Z. Bern, A. De Freitas, L.J. Dixon, A. Ghinculov and H.L. Wong, "QCD and QED corrections to light-by-light scattering," JHEP 0111, 031 (2001) [arXiv:hep-ph/0109079].

[9] Z. Bern, A. De Freitas and L. Dixon, "Two-loop helicity amplitudes for gluon-gluon scattering in QCD and supersymmetric Yang-Mills theory," JHEP 0203, 018 (2002) [arXiv:hep-ph/0201161].

[10] L.W. Garland, T. Gehrmann, E.W.N. Glover, A. Koukoutsakis and E. Remiddi, "The two-loop QCD matrix element for $e^{+} e^{-} \rightarrow 3$ jets," Nucl. Phys. B 627, 107 (2002) [arXiv:hep-ph/0112081].

[11] L.W. Garland, T. Gehrmann, E.W.N. Glover, A. Koukoutsakis and E. Remiddi, "Two-loop QCD helicity amplitudes for $e^{+} e^{-} \rightarrow 3$ jets," Nucl. Phys. B 642, 227 (2002) [arXiv:hep-ph/0206067];

S. Moch, P. Uwer and S. Weinzierl, "Two-loop amplitudes for $e^{+} e^{-} \rightarrow q \bar{q} g$ : The $N_{f}$-contribution," Acta Phys. Polon. B 33, 2921 (2002) [arXiv:hep-ph/0207167].

[12] F.V. Tkachov, "A theorem on analytical calculability of four-loop renormalization group functions," Phys. Lett. B 100, 65 (1981);

K.G. Chetyrkin and F.V. Tkachov, "Integration by parts: the algorithm to calculate beta functions in 4 loops," Nucl. Phys. B 192, 159 (1981). 
[13] V.A. Smirnov, "Analytical result for dimensionally regularized massless on-shell double box," Phys. Lett. B460, 397 (1999) [arXiv:hep-ph/9905323].

[14] J.B. Tausk, "Non-planar massless two-loop Feynman diagrams with four on-shell legs," Phys. Lett. B469, 225 (1999) [arXiv:hep-ph/9909506].

[15] T. Gehrmann and E. Remiddi, "Differential equations for two-loop four-point functions," Nucl. Phys. B 580, 485 (2000) [arXiv:hep-ph/9912329].

[16] C. Anastasiou, T. Gehrmann, C. Oleari, E. Remiddi and J.B. Tausk, "The tensor reduction and master integrals of the two-loop massless crossed box with light-like legs," Nucl. Phys. B580, 577 (2000) [arXiv:hep-ph/0003261].

[17] V.A. Smirnov and O.L. Veretin, "Analytical results for dimensionally regularized massless on-shell double boxes with arbitrary indices and numerators," Nucl. Phys. B566, 469 (2000) [arXiv:hep-ph/9907385].

[18] C. Anastasiou, E.W.N. Glover and C. Oleari, "Application of the negative-dimension approach to massless scalar box integrals," Nucl. Phys. B565, 445 (2000) [arXiv:hep-ph/9907523]; C. Anastasiou, E.W.N. Glover and C. Oleari, "The two-loop scalar and tensor pentabox graph with light-like legs," Nucl. Phys. B575, 416 (2000), err. ibid. B585, 763 (2000) [arXiv:hep-ph/9912251].

[19] S. Catani, "The singular behaviour of QCD amplitudes at two-loop order," Phys. Lett. B427, 161 (1998) [arXiv:hep-ph/9802439].

[20] E.W.N. Glover, "Progress in NNLO calculations for scattering processes," arXiv:hep-ph/0211412.

[21] F. Aversa, P. Chiappetta, M. Greco and J.P. Guillet, "Higher order corrections to QCD jets," Phys. Lett. B 210, 225 (1988); "Jet production in hadronic collisions to $\mathcal{O}\left(\alpha_{s}^{3}\right)$," Z. Phys. C 46, 253 (1990).

[22] S.D. Ellis, Z. Kunszt and D.E. Soper, "The one-jet inclusive cross section at $\mathcal{O}\left(\alpha_{s}^{3}\right)$ : quarks and gluons," Phys. Rev. Lett. 64, 2121 (1990); "Two jet production in hadron collisions at $\mathcal{O}\left(\alpha_{s}^{3}\right)$ in QCD," Phys. Rev. Lett. 69, 1496 (1992).

[23] W.T. Giele, E.W.N. Glover and D.A. Kosower, "Higher order corrections to jet cross sections in hadron colliders," Nucl. Phys. B 403, 633 (1993) [arXiv:hep-ph/9302225].

[24] A.D. Martin, R.G. Roberts, W.J. Stirling and R.S. Thorne, "Estimating the effect of NNLO contributions on global parton analyses," Eur. Phys. J. C 18, 117 (2000) [arXiv:hep-ph/0007099]; "NNLO global parton analysis," Phys. Lett. B 531, 216 (2002) [arXiv:hep-ph/0201127].

[25] W.L. van Neerven and A. Vogt, "NNLO evolution of deep-inelastic structure functions: The non-singlet case," Nucl. Phys. B 568, 263 (2000) [arXiv:hep-ph/9907472];

"NNLO evolution of deep-inelastic structure functions: The singlet case," Nucl. Phys. B 588, 345 (2000) [arXiv:hep-ph/0006154];

"Improved approximations for the three-loop splitting functions in QCD," Phys. Lett. B 490, 111 (2000) [arXiv:hep-ph/0007362];

S. Moch, J.A.M. Vermaseren and A. Vogt, "Non-singlet structure functions at three loops: Fermionic contributions," Nucl. Phys. B 646, 181 (2002) [arXiv:hep-ph/0209100]. 
[26] S. Alekhin, "Extraction of parton distributions and $\alpha_{s}$ from DIS data within the Bayesian treatment of systematic errors," Eur. Phys. J. C 10, 395 (1999) [arXiv:hep-ph/9611213]; W.T. Giele, S.A. Keller and D.A. Kosower, "Parton distributions with errors," in La Thuile 1999, Results and perspectives in particle physics;

D. Stump et al., "Uncertainties of predictions from parton distribution functions. I: The Lagrange multiplier method," Phys. Rev. D 65, 014012 (2002) [arXiv:hep-ph/0101051]; "Uncertainties of predictions from parton distribution functions. II: The Hessian method," Phys. Rev. D 65, 014013 (2002) [arXiv:hep-ph/0101032];

W.T. Giele, S.A. Keller and D.A. Kosower, "Parton distribution function uncertainties," arXiv:hep-ph/0104052;

A.D. Martin, R.G. Roberts, W.J. Stirling and R.S. Thorne, "Uncertainties of predictions from parton distributions. I: Experimental errors," arXiv:hep-ph/0211080.

[27] N. Kidonakis, "Resummation for heavy quark and jet cross sections," Int. J. Mod. Phys. A 15, 1245 (2000) [arXiv:hep-ph/9902484];

N. Kidonakis and J.F. Owens, "Effects of higher-order threshold corrections in high- $E_{T}$ jet production," Phys. Rev. D 63, 054019 (2001) [arXiv:hep-ph/0007268].

[28] F.A. Berends and W. Giele, "The six gluon process as an example of Weyl-Van Der Waerden spinor calculus," Nucl. Phys. B 294, 700 (1987);

M.L. Mangano, S. Parke and Z. Xu, "Duality and multi-gluon scattering," Nucl. Phys. B 298, 653 (1988).

[29] M.L. Mangano and S.J. Parke, "Multiparton amplitudes in gauge theories," Phys. Rept. 200, 301 (1991).

[30] Z. Bern, L.J. Dixon and D.A. Kosower, "One-loop corrections to five-gluon amplitudes," Phys. Rev. Lett. 70, 2677 (1993) [arXiv:hep-ph/9302280];

Z. Kunszt, A. Signer and Z. Trócsányi, "One-loop radiative corrections to the helicity amplitudes of QCD processes involving four quarks and one gluon," Phys. Lett. B 336, 529 (1994) [arXiv:hep-ph/9405386].

[31] Z. Bern, L.J. Dixon and D.A. Kosower, "One-loop corrections to two-quark three-gluon amplitudes," Nucl. Phys. B 437, 259 (1995) [arXiv:hep-ph/9409393].

[32] F.A. Berends, R. Kleiss, P. De Causmaecker, R. Gastmans and T.T. Wu, "Single bremsstrahlung processes in gauge theories," Phys. Lett. B 103, 124 (1981); P. De Causmaecker, R. Gastmans, W. Troost and T.T. Wu, "Helicity amplitudes for massless QED," Phys. Lett. B 105, 215 (1981);

Z. Xu, D. Zhang and L. Chang, "Helicity amplitudes for multiple bremsstrahlung in massless nonabelian gauge theories," Nucl. Phys. B 291, 392 (1987).

[33] M.T. Grisaru, H.N. Pendleton and P. van Nieuwenhuizen, "Supergravity and the S matrix," Phys. Rev. D 15, 996 (1977);

M.T. Grisaru and H.N. Pendleton, "Some properties of scattering amplitudes in supersymmetric theories," Nucl. Phys. B 124, 81 (1977);

S.J. Parke and T.R. Taylor, "Perturbative QCD utilizing extended supersymmetry," Phys. Lett. B 157, 81 (1985), err. ibid. 174B, 465 (1985).

[34] Z. Bern, A. De Freitas, L. Dixon and H.L. Wong, "Supersymmetric regularization, two-loop QCD amplitudes and coupling shifts," Phys. Rev. D 66, 085002 (2002)

[arXiv:hep-ph/0202271]. 
[35] Z. Bern, L.J. Dixon, D.C. Dunbar and D.A. Kosower, "One-loop n-point gauge theory amplitudes, unitarity and collinear limits," Nucl. Phys. B425, 217 (1994) [arXiv:hep-ph/9403226].

[36] Z. Bern, L.J. Dixon and D.A. Kosower, "Progress in one-loop QCD computations," Ann. Rev. Nucl. Part. Sci. 46, 109 (1996) [arXiv:hep-ph/9602280].

[37] E.A. Kuraev, L.N. Lipatov and V.S. Fadin, "Multi-reggeon processes in the Yang-Mills theory," Sov. Phys. JETP 44, 443 (1976) [Zh. Eksp. Teor. Fiz. 71, 840 (1976)].

[38] V. Del Duca and E.W.N. Glover, "The high energy limit of QCD at two loops," JHEP 0110, 035 (2001) [arXiv:hep-ph/0109028];

A.V. Bogdan, V. Del Duca, V.S. Fadin and E.W.N. Glover, "The quark Regge trajectory at two loops," JHEP 0203, 032 (2002) [arXiv:hep-ph/0201240].

[39] G. Sterman and M.E. Tejeda-Yeomans, "Multi-loop amplitudes and ressumation," Phys. Lett. B 552, 48 (2003) [arXiv:hep-ph/0210130].

[40] J. Soffer and J.M. Virey, "Testing various polarized parton distributions at RHIC," Nucl. Phys. B 509, 297 (1998) [arXiv:hep-ph/9706229].

[41] D. de Florian, S. Frixione, A. Signer and W. Vogelsang, "Next-to-leading order jet cross sections in polarized hadronic collisions," Nucl. Phys. B 539, 455 (1999) [arXiv:hep-ph/9808262].

[42] E.W.N. Glover and M.E. Tejeda-Yeomans, "Two-loop QCD helicity amplitudes for massless quark-massless gauge boson scattering," arXiv:hep-ph/0304169.

[43] J.C. Collins, Renormalization: an introduction to renormalization group, and the operator-product expansion, Cambridge Monographs on Mathematical Physics (Cambridge Univ. Press, 1984).

[44] R.K. Ellis and J.C. Sexton, "QCD Radiative corrections to parton-parton scattering," Nucl. Phys. B 269, 445 (1986).

[45] G. 't Hooft and M.J.G. Veltman, "Regularization and renormalization of gauge fields," Nucl. Phys. B44, 189 (1972).

[46] Z. Bern and D.A. Kosower, "The computation of loop amplitudes in gauge theories," Nucl. Phys. B379, 451 (1992).

[47] W. Siegel, "Supersymmetric dimensional regularization via dimensional reduction," Phys. Lett. B84, 193 (1979);

D.M. Capper, D.R.T. Jones and P. van Nieuwenhuizen, "Regularization by dimensional reduction of supersymmetric and nonsupersymmetric gauge theories," Nucl. Phys. B167, 479 (1980);

I. Jack, D.R.T. Jones and K.L. Roberts, "Equivalence of dimensional reduction and dimensional regularization," Z. Phys. C63, 151 (1994) [arXiv:hep-ph/9401349].

[48] T. Binoth, E.W.N. Glover, P. Marquard and J.J. van der Bij, "Two-loop corrections to light-by-light scattering in supersymmetric QED," JHEP 0205, 060 (2002) [arXiv:hep-ph/0202266].

[49] S. Catani and M. Grazzini, "The soft-gluon current at one-loop order," Nucl. Phys. B 591, 435 (2000) [arXiv:hep-ph/0007142]. 
[50] Z. Kunszt, A. Signer and Z. Trócsányi, "One-loop helicity amplitudes for all $2 \rightarrow 2$ processes in QCD and $N=1$ supersymmetric Yang-Mills theory," Nucl. Phys. B411, 397 (1994) [arXiv:hep-ph/9305239].

[51] S. Catani, M.H. Seymour and Z. Trócsányi, "Regularization scheme independence and unitarity in QCD cross sections," Phys. Rev. D55, 6819 (1997) [arXiv:hep-ph/9610553].

[52] C. Anastasiou, E.W.N. Glover and M.E. Tejeda-Yeomans, private communications.

[53] C. Anastasiou, E.W.N. Glover and M.E. Tejeda-Yeomans, "Two-loop QED and QCD corrections to massless fermion boson scattering," Nucl. Phys. B 629, 255 (2002) [arXiv:hep-ph/0201274].

[54] P. Nogueira, "Automatic Feynman graph generation," J. Comput. Phys. 105, 279 (1993).

[55] J.A.M. Vermaseren, "New features of FORM," arXiv:math-ph/0010025.

[56] Z. Bern and A. G. Morgan, "Massive Loop Amplitudes from Unitarity," Nucl. Phys. B 467, 479 (1996) [arXiv:hep-ph/9511336].

[57] K.S. Kölbig, J.A. Mignaco and E. Remiddi, "On Nielsen's generalized polylogarithms and their numerical calculation," B.I.T. 10, 38 (1970).

[58] L. Lewin, Dilogarithms and Associated Functions (Macdonald, 1958).

[59] Z. Bern and G. Chalmers, "Factorization in one-loop gauge theory," Nucl. Phys. B 447, 465 (1995) [arXiv:hep-ph/9503236];

Z. Bern, V. Del Duca and C.R. Schmidt, "The infrared behavior of one-loop gluon amplitudes at next-to-next-to-leading order," Phys. Lett. B 445, 168 (1998) [arXiv:hep-ph/9810409];

D.A. Kosower and P. Uwer, "One-loop splitting amplitudes in gauge theory," Nucl. Phys. B 563, 477 (1999) [arXiv:hep-ph/9903515];

Z. Bern, V. Del Duca, W.B. Kilgore and C.R. Schmidt, "The infrared behavior of one-loop QCD amplitudes at next-to-next-to-leading order," Phys. Rev. D 60, 116001 (1999) [arXiv:hep-ph/9903516].

[60] J.F. Gunion and Z. Kunszt, "Improved analytic techniques for tree graph calculations and the $g g q \bar{q} \ell \bar{\ell}$ subprocess," Phys. Lett. B 161, 333 (1985).

[61] Z. Bern and D.A. Kosower, "Color decomposition of one-loop amplitudes in gauge theories," Nucl. Phys. B 362, 389 (1991).

[62] Z. Bern, L. Dixon and C. Schmidt, "Isolating a light Higgs boson from the di-photon background at the LHC," Phys. Rev. D 66, 074018 (2002) [arXiv:hep-ph/0206194]; L. Dixon and M.S. Siu, "Resonance-continuum interference in the di-photon Higgs signal at the LHC," arXiv:hep-ph/0302233.

[63] D.A. Kosower, "Multiple singular emission in gauge theories," arXiv:hep-ph/0212097;

S. Weinzierl, "Subtraction terms at NNLO," arXiv:hep-ph/0302180. 